\title{
Synthesis and evaluation of novel 7- and 8-aminophenoxazinones for the detection of $\beta$-alanine aminopeptidase activity and the reliable identification of Pseudomonas aeruginosa in clinical samples
}

\footnotetext{
Alexandre F. Bedernjak, ${ }^{\mathrm{a}}$ Andrey V. Zaytsev, ${ }^{\mathrm{b}}$ Michèle Babolat, ${ }^{\mathrm{b}}$ Marie Cellier, ${ }^{\mathrm{b}}$ The Late Arthur L. James, ${ }^{c}$ Sylvain Orenga, ${ }^{\mathrm{b}}$ John D. Perry, ${ }^{\mathrm{d}}$ Paul W. Groundwater, ${ }^{\mathrm{a} \dagger}$ and Rosaleen J. Anderson $^{\mathrm{a} *}$

${ }^{a}$ Sunderland Pharmacy School, University of Sunderland, Sunderland SR1 3SD, UK; *corresponding author; email: roz.anderson@sunderland.ac.uk; Tel: +441915152591

${ }^{b}$ Department of Microbiology, bioMérieux, 38390 La Balme-les-Grottes, France;

${ }^{c}$ School of Life Sciences, Northumbria University, Newcastle upon Tyne, NE1 8ST, UK;

${ }^{d}$ Department of Microbiology, Freeman Hospital, Newcastle upon Tyne NE7 7DN, UK.
}

\section{Supporting Information}

\section{Table of contents}

1.1 Chemicals, materials and methods

1.2 Synthesis of dimethoxyphenols $\mathbf{4 e}$ and $\mathbf{4 f}$

1.3 Synthesis of diphenylethers 6a-f

1.4 Preparation of 2,5-dinitro-1-fluorobenzene $\mathbf{1 3}$

1.5 Synthesis of diphenylethers $\mathbf{1 4 a - b}$

1.6 Synthesis of dihydroxydiphenylethers 7a-b

1.7 Synthesis of dinitrobenzophenones $8 \mathbf{b}-8 \mathbf{e}$ and $\mathbf{1 5 a}-\mathbf{b}$

1.9 Synthesis of 8-amino-alkyl-3H-phenoxazin-3-ones 2a-f and

\footnotetext{
${ }^{\dagger}$ Now at Faculty of Pharmacy, The University of Sydney, Sydney NSW 2006, Australia.
} 
1.10 Synthesis of 7- $N$ - and 8- $N-\left(N^{\prime}-\right.$ butoxycarbonyl- $\beta$-alanyl)amino-3H-phenoxazin-3-ones 20a-b and 21a-f

1.11 Synthesis of 7- $N$ - and 8- $N$-( $\beta$-alanyl)amino-3H-phenoxazin-3-one TFA salts 22a-b and 23a-f

1.12 References

1.13 Figures: Selected LC-MS and NMR spectra of key intermediates and products

Figure S1. HPLC-MS Spectra of $\mathbf{2 b}$

Figure S2. HPLC-MS Spectra of $\mathbf{2 c}$.

Figure S3. HPLC-MS Spectra of 22a

Figure S4. HPLC-MS Spectra of 23b

Figure S5. HPLC-MS Spectra of 23c

Figure S6. HPLC-MS Spectra of 23d

Figure S7. HPLC-MS Spectra of 23e

Figure S8. HPLC-MS Spectra of $\mathbf{2 3 f}$

Figure S9. ${ }^{1} \mathrm{H}$ NMR spectrum of $22 \mathbf{a}$ in $\mathrm{d}_{6}-\mathrm{DMSO}$ at $50^{\circ} \mathrm{C}$

Figure S10. ${ }^{1} \mathrm{H}$ NMR spectrum of $22 \mathbf{b}$ in $\mathrm{d}_{6}-\mathrm{DMSO}$ at $30^{\circ} \mathrm{C}$

Figure S11. ${ }^{1} \mathrm{H}$ NMR spectrum of $23 a$ in $d_{6}$-DMSO at $30^{\circ} \mathrm{C}$

Figure S12. ${ }^{1} \mathrm{H}$ NMR spectrum of $\mathbf{2 3 b}$ in $\mathrm{d}_{6}$-DMSO at $30^{\circ} \mathrm{C}$

Figure S13. ${ }^{1} \mathrm{H}$ NMR spectrum of $23 \mathrm{c}$ in $\mathrm{d}_{6}$-DMSO at $30^{\circ} \mathrm{C}$

Figure S14. ${ }^{1} \mathrm{H}$ NMR spectrum of $23 d$ in $\mathrm{d}_{6}$-DMSO at $30^{\circ} \mathrm{C}$

Figure S15. ${ }^{1} \mathrm{H}$ NMR spectrum of $23 \mathrm{e}$ in $\mathrm{d}_{6}$-DMSO at $50^{\circ} \mathrm{C}$

Figure S16. ${ }^{1} \mathrm{H}$ NMR spectrum of $23 f$ in $\mathrm{d}_{6}$-DMSO at $50^{\circ} \mathrm{C}$ 


\subsection{Chemicals, materials and methods}

All commercially available reagents and solvents were obtained from Sigma-Aldrich, Fluka or Riedel-de-Haan and were used without further purification. All synthesized compounds were pure to $\geq 95 \%$ by at least one analytical method, except $23 \mathbf{a}$, which had approx. $90 \%$ purity. Melting points were recorded on a Reichart-Kofler hot-stage microscope apparatus and are uncorrected. Infrared spectra were recorded in the range $4000-600 \mathrm{~cm}^{-1}$ using a Perkin Elmer Spectrum BX FT-IR spectrophotometer. NMR spectra were obtained using a Bruker Ultrashield 300 spectrometer at $300 \mathrm{MHz}$ for ${ }^{1} \mathrm{H}$ spectra or at $75 \mathrm{MHz}$ for ${ }^{13} \mathrm{C}$ spectra. Lowresolution mass spectra were recorded on a Bruker Esquire 3000plus analyser using an electrospray source in either positive or negative ion mode. High resolution accurate mass measurements were collected by the EPSRC National Mass Spectrometry Facility at Swansea University. Elemental analyses were performed using an Exeter Analytical CE-440 Elemental Analyzer. Thin layer chromatography was performed on Merck silica gel $60 \mathrm{~F}_{254}$. Fisons silica gel, 60 (35-70 micron) was used for flash chromatography; the samples were pre-absorbed onto silica 60 (35-70 micron).

\subsection{Synthesis of dimethoxyphenols $4 \mathrm{e}$ and $4 \mathrm{f}$}

2,5-Dimethoxybenzaldehydes 3a-d and 2,5-dimethoxyphenols 4a-d were prepared according to procedures previously described ${ }^{1}$, while phenols $\mathbf{4 e}$ and $\mathbf{4 f}$ were accessed by introduction of alkyl or aryl substituents (Scheme S1) in order to increase the lipophilic properties of the final chromogen. 2,5-Dimethoxy-4-pentylphenol 4e was prepared from commercially available 1-bromo-2,5-dimethoxybenzene $\mathbf{2 4 a ( S c h e m e ~ S 1 ) . ~ L i t h i u m - h a l o g e n ~ e x c h a n g e , ~ i n ~}$

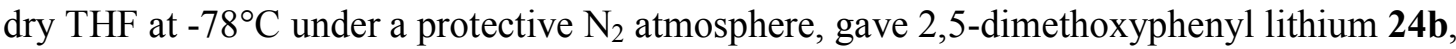
and the addition of 1-bromopentane followed by refluxing of the resulting mixture yielded 1,4-dimethoxy-2-pentylbenzene 25. Formylation of ether 25 using the Duff conditions ${ }^{2}$ gave 2,5-dimethoxy-4-pentylbenzaldehyde 3e in good yield. 2,5-Dimethoxy-4-pentylphenol 4e was finally obtained via the Baeyer-Villiger oxidation of $\mathbf{3 e}$ and hydrolysis of the resulting formate ester (Scheme S1).

2,5-Dimethoxy-3-phenylphenol $\mathbf{4 f}$ was prepared from the commercially available 2-hydroxy5-methoxybenzaldehyde 26. Regioselective bromination, to give the tri-substituted benzaldehyde $\mathbf{2 7}$, followed by methylation gave 3-bromo-2,5-dimethoxybenzaldehyde $\mathbf{2 8}^{3}$. Suzuki coupling of benzaldehyde $\mathbf{2 8}$ with phenylboronic acid, using the conditions described 
by Yonezawa et $a l .{ }^{4}$, gave 2,5-dimethoxy-3-phenylbenzaldehyde 3f, the Baeyer-Villiger oxidation of which provided 2,5-dimethoxy-3-phenylphenol $4 \mathbf{f}$ in good yield (Scheme S1).

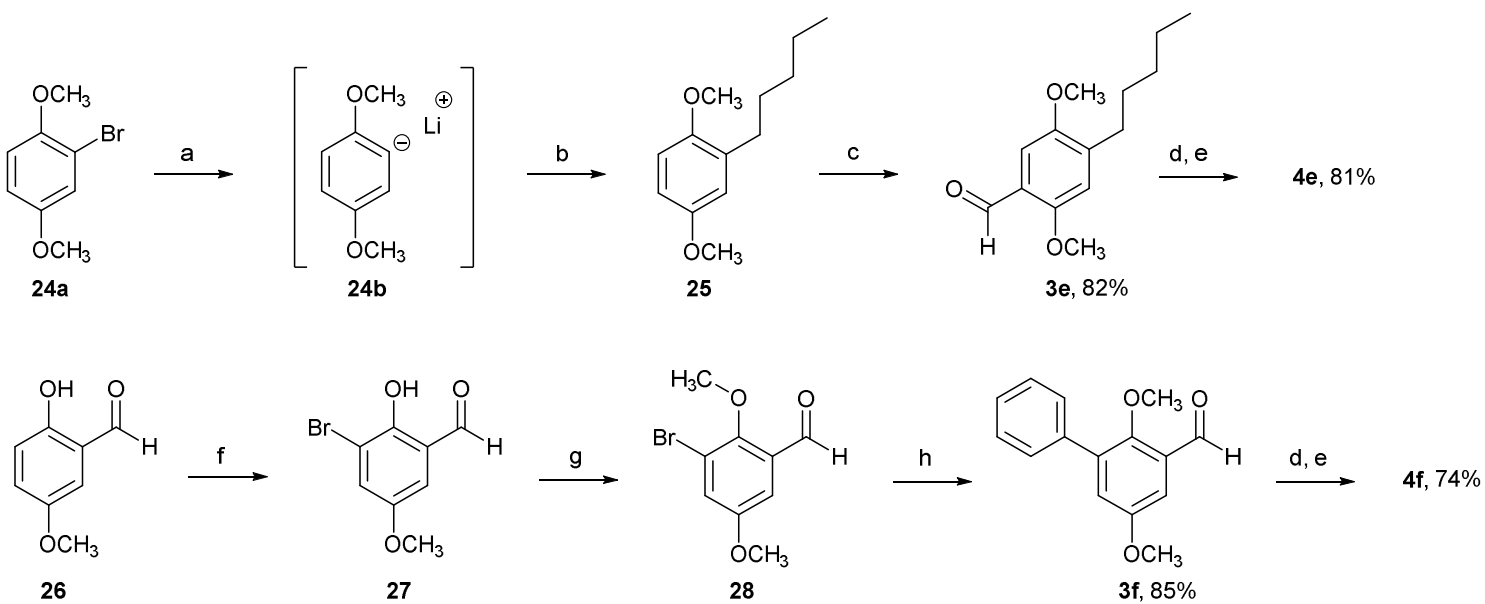

Scheme S1. Synthesis of substituted dimethoxyphenols $4 \mathbf{e}$ and $\mathbf{4 f}$. Reagents and conditions: [a] 2.5M $n$-BuLi, dry THF, TMEDA, $\mathrm{N}_{2}$ atm., $-78^{\circ} \mathrm{C}, 1 \mathrm{~h}$; [b] 1-bromopentane, $-78^{\circ} \mathrm{C}$ to reflux, $15 \mathrm{~h}$; [c] TFA, urotropine, reflux, 12h; [d] $m$-CPBA, DCM, R.T., 24h; [e] 10\% aq. NaOH, MeOH; [f] $\mathrm{Br}_{2}$, AcOH, AcONa, R.T.; [g] DMF, $\mathrm{K}_{2} \mathrm{CO}_{3}$, $(\mathrm{MeO})_{2} \mathrm{SO}_{2} ;[\mathrm{h}] 2 \mathrm{M} \mathrm{Na}_{2} \mathrm{CO}_{3}, \mathrm{Pd}\left(\mathrm{PPh}_{3}\right)_{4}$, phenyl boronic acid, DME, EtOH, $100^{\circ} \mathrm{C}, 18 \mathrm{~h}$.

\subsubsection{1,4-Dimethoxy-2-pentylbenzene 25}

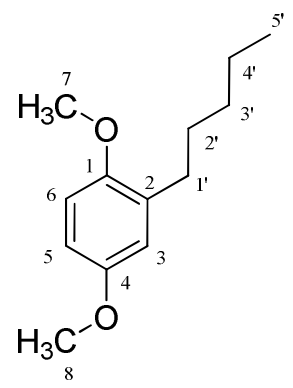

A flame dried round bottomed flask, flushed with $\mathrm{N}_{2}$, was charged with 1-bromo-2,5dimethoxybenzene 24a $(4.0 \mathrm{~mL}, 5.78 \mathrm{~g}, 26.63 \mathrm{mmol})$, TMEDA $(8.03 \mathrm{~mL}, 6.18 \mathrm{~g}, 53.20 \mathrm{mmol})$ and dry THF $(150 \mathrm{~mL})$. The resulting solution was cooled to $-75^{\circ} \mathrm{C}$, then a $2.5 \mathrm{M}$ hexane solution of $n$-BuLi $(24 \mathrm{~mL}, 60.00 \mathrm{mmol})$ was added dropwise and the reaction mixture stirred a further $1 \mathrm{~h}$ at $-75^{\circ} \mathrm{C}$. Neat 1-bromopentane $(18.65 \mathrm{~mL}, 150.0 \mathrm{mmol})$ was added dropwise and the resulting solution left with stirring at $-75^{\circ} \mathrm{C}$ under $\mathrm{N}_{2}$ flow for $1 \mathrm{~h}$, allowed to warm to $\mathrm{rt}$, and then refluxed for a period of $15 \mathrm{~h}$. The formation of a white precipitate was noted after the reflux. The resulting mixture was allowed to cool to $\mathrm{rt}$, quenched with water $(200 \mathrm{~mL})$ and extracted with $\mathrm{Et}_{2} \mathrm{O}(2 \times 200 \mathrm{~mL})$. The combined organic layers were successively washed with water $(200 \mathrm{~mL})$ and brine $(200 \mathrm{~mL})$, and dried $\left(\mathrm{MgSO}_{4}\right)$. The solvent was removed in 
vacuo and the residue purified by column chromatography eluting with petroleum ether (60$\left.80^{\circ} \mathrm{C}\right): \mathrm{Et}_{2} \mathrm{O}$ (95:5). 1,4-Dimethoxy-2-pentylbenzene 25 was isolated as a pale yellow oil (4.75g, 22.80mmol, 85.7\%), bp $81-82^{\circ} \mathrm{C} / 0.2 \mathrm{mbar}$ [lit. bp $114^{\circ} \mathrm{C} / 6.7 \mathrm{mbar}^{5}$ ]; $\mathrm{m} / \mathrm{z} 209.0(M H)^{+}$; (found: $\mathrm{C}, 74.65 ; \mathrm{H}, 9.5 \%$. $\mathrm{C}_{13} \mathrm{H}_{20} \mathrm{O}_{2}$ requires $\left.\mathrm{C}, 75.0 ; \mathrm{H}, 9.7 \%\right) ; v_{\max } / \mathrm{cm}^{-1} 2832-2928(\mathrm{C}-\mathrm{H}$ ), $1498(\mathrm{C}=\mathrm{C}), 1219(\mathrm{C}-\mathrm{O}), 1048(\mathrm{C}-\mathrm{O}) ; \delta_{H}\left(300 \mathrm{MHz}, \mathrm{CDCl}_{3}\right) 0.81(3 \mathrm{H}, \mathrm{t}, J=6.9 \mathrm{~Hz}, 5$ '-H), 1.24-1.28 (4H, m, 3'-H and 4'-H), 1.45-1.52 (2H, m, 2'-H), 2.49 (2H, t, $\left.J=7.5 \mathrm{~Hz}, 1^{\prime}-\mathrm{H}\right), 3.67$ $\left(3 \mathrm{H}, \mathrm{s}, 1-\mathrm{OCH}_{3}\right.$ or $\left.4-\mathrm{OCH}_{3}\right), 3.68\left(3 \mathrm{H}, \mathrm{s}, 1-\mathrm{OCH}_{3}\right.$ or $\left.4-\mathrm{OCH}_{3}\right), 6.59(1 \mathrm{H}, \mathrm{dd}, J=8.7,3.0 \mathrm{~Hz}$, $5-\mathrm{H}), 6.65(1 \mathrm{H}, \mathrm{d}, J=3.0 \mathrm{~Hz}, 3-\mathrm{H}), 6.67(1 \mathrm{H}, \mathrm{d}, J=8.7 \mathrm{~Hz}, 6-\mathrm{H}) ; \delta_{C}\left(75 \mathrm{MHz}, \mathrm{CDCl}_{3}\right) 14.1$ $\left(\mathrm{CH}_{3}, 5^{\prime}-\mathrm{C}\right), 22.6\left(\mathrm{CH}_{2}, 3^{\prime}-\mathrm{C}\right.$ or 4'-C), $29.6\left(\mathrm{CH}_{2}, 2^{\prime}-\mathrm{C}\right), 30.3\left(\mathrm{CH}_{2}, 1^{\prime}-\mathrm{C}\right), 31.8\left(\mathrm{CH}_{2}, 3^{\prime}-\mathrm{C}\right.$ or 4'-C), $55.7\left(\mathrm{OCH}_{3}, 8-\mathrm{C}\right), 56.0\left(\mathrm{OCH}_{3}, 7-\mathrm{C}\right), 110.6(\mathrm{CH}, 5-\mathrm{C}), 111.3(\mathrm{CH}, 6-\mathrm{C}), 116.3(\mathrm{CH}, 3-$ C), 132.8 (quat., 2-C), 151.9 (quat., 1-C), 153.55 (quat., 4-C).

\subsubsection{2,5-Dimethoxy-4-pentylbenzaldehyde 3e}

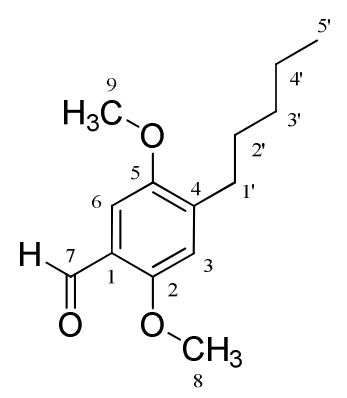

In a $250 \mathrm{~mL}$ round bottomed flask equipped with a reflux condenser, 2,5-dimethoxy-1pentylbenzene $25(4.49 \mathrm{~g}, 21.56 \mathrm{mmol})$ was dissolved in TFA $(120 \mathrm{~mL})$ and hexamethylene tetramine $(6.04 \mathrm{~g}, 43.12 \mathrm{mmol})$ was added to the resulting solution, which was gently refluxed $\left(\mathrm{t}_{\text {ext. }}=95^{\circ} \mathrm{C}\right)$ for $48 \mathrm{~h}$. The solution was allowed to cool to rt; most of the TFA was removed in vacuo and the residue diluted with water $(50 \mathrm{~mL})$ and neutralised by the portion-wise addition of solid $\mathrm{Na}_{2} \mathrm{CO}_{3}$. When no more evolution of $\mathrm{CO}_{2}$ was observed, the resulting mixture was diluted with another $50 \mathrm{~mL}$ of water and then extracted with $\mathrm{Et}_{2} \mathrm{O}(3 \times 200 \mathrm{~mL})$, washed with brine $(200 \mathrm{~mL})$ and dried $\left(\mathrm{MgSO}_{4}\right)$. The solvent was removed in vacuo and the residue purified by column chromatography, eluting with a mixture of petroleum ether $\left(60-80^{\circ} \mathrm{C}\right)$ : $\mathrm{Et}_{2} \mathrm{O}$ (80:20). 2,5-Dimethoxy-4-pentylbenzaldehyde 3e was isolated as a pale yellow oil (4.19g, 17.73mmol, 82.4\%); bp $119-120^{\circ} \mathrm{C} / 0.2 \mathrm{mbar}$ [lit. bp $201-205^{\circ} \mathrm{C} / 27 \mathrm{mbar}^{6}$ ]; (found $\mathrm{C}$, 71.0; $\mathrm{H}, 8.5 . \mathrm{C}_{14} \mathrm{H}_{20} \mathrm{O}_{3}$ requires $\left.\mathrm{C}, 71.2 ; \mathrm{H}, 8.5 \%\right) ; \mathrm{m} / \mathrm{Z} 237.0(M H)^{+}, 259.0(M N a)^{+} ; v_{\max } / \mathrm{cm}^{-1}$ $1675(\mathrm{C}=\mathrm{O}), 1610(\mathrm{C}=\mathrm{C}), 1210$ and $1040(\mathrm{C}-\mathrm{O}) ; \delta_{H}\left(300 \mathrm{MHz}, \mathrm{CDCl}_{3}\right) 0.94(3 \mathrm{H}, \mathrm{t}, J=$ $\left.6.9 \mathrm{~Hz}, 5^{\prime}-\mathrm{H}\right), 1.35-1.42\left(4 \mathrm{H}, \mathrm{m}, 3^{\prime}-\mathrm{H}\right.$ and $\left.4^{\prime}-\mathrm{H}\right), 1.60-1.65\left(2 \mathrm{H}, \mathrm{m}, 2^{\prime}-\mathrm{H}\right), 2.67(2 \mathrm{H}, \mathrm{t}, J=$ 
7.5Hz, 1'-H), $3.85\left(3 \mathrm{H}, \mathrm{s}, 2-\mathrm{OCH}_{3}\right), 3.92\left(3 \mathrm{H}, \mathrm{s}, 5-\mathrm{OCH}_{3}\right), 6.82(1 \mathrm{H}, \mathrm{s}, 3-\mathrm{H}), 7.29(1 \mathrm{H}, \mathrm{s}, 6-$ $\mathrm{H}), 10.43(1 \mathrm{H}, \mathrm{s}, \mathrm{CHO}, 7-\mathrm{H}) ; \delta_{C}\left(75 \mathrm{MHz}, \mathrm{CDCl}_{3}\right) 14.0\left(\mathrm{CH}_{3}, 5^{\prime}-\mathrm{C}\right), 22.5\left(\mathrm{CH}_{2}, 3^{\prime}-\mathrm{C}\right.$ or 4'-C), $29.2\left(\mathrm{CH}_{2}, 2^{\prime}-\mathrm{C}\right), 31.0\left(\mathrm{CH}_{2}, 1^{\prime}-\mathrm{C}\right), 31.8\left(\mathrm{CH}_{2}, 3^{\prime}-\mathrm{C}\right.$ or 4 '-C $), 55.8\left(\mathrm{OCH}_{3}, 2-\mathrm{OCH}_{3}\right), 56.2$ $\left(\mathrm{OCH}_{3}, 5-\mathrm{OCH}_{3}\right), 108.1(\mathrm{CH}, 6-\mathrm{C}), 113.8(\mathrm{CH}, 3-\mathrm{C}), 122.9$ (quat., 1-C), 141.2 (quat., 4-C), 151.8 (quat., 2-C), 156.7 (quat., 5-C), 189.15 (CHO, 7-C).

\subsubsection{3-Bromo-2-hydroxy-5-methoxybenzaldehyde 27}

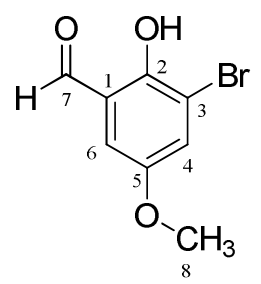

In a 3 necked round bottomed flask equipped with a thermometer and a pressure equalizing dropping funnel, 2-hydroxy-5-methoxybenzaldehyde 26 (11.51g, 75.65mmol) was dissolved in glacial AcOH $(300 \mathrm{~mL})$. To this solution was added AcONa $(10.34 \mathrm{~g}, 126.05 \mathrm{mmol})$ and the resulting solution was cooled with a water/ice bath. A solution of $\mathrm{Br}_{2}(4.91 \mathrm{~mL}, 95.33 \mathrm{mmol})$ in $\mathrm{AcOH}(50 \mathrm{~mL})$ was then introduced into the pressure equalizing dropping funnel and added dropwise to the 2-hydroxy-4-methoxybenzaldehyde solution. Upon completion of the addition (1h), the ice/water bath was removed and the resulting orange solution left with stirring overnight. The reaction was quenched with sodium thiosulfate $\left(10 \%, \mathrm{Na}_{2} \mathrm{~S}_{2} \mathrm{O}_{3}\right)$, resulting in the instantaneous formation of a yellow precipitate, which was recovered by filtration, washed several times with water and dried. The filtrate was extracted with DCM $(4 \times 200 \mathrm{~mL})$ and the yellow precipitate dissolved in the combined organic layers, which were washed with water (2 $\times 150 \mathrm{~mL})$ and brine $(150 \mathrm{~mL})$, and dried $\left(\mathrm{MgSO}_{4}\right)$. The solvent was evaporated in vacuo yielding 3-bromo-2-hydroxy-5-methoxybenzaldehyde $\mathbf{2 7}$ as a yellow crystalline solid (12.86g, 55.66mmol, 75.1\%). Small crescent-like yellow plates were obtained from aq. EtOH; mp $108-109^{\circ} \mathrm{C}$ [lit.: $108^{\circ} \mathrm{C}$ (from $\left.\mathrm{EtOH}\right)^{3}$ ]; (found $\mathrm{C}, 41.6 ; \mathrm{H}, 2.95 \% . \mathrm{C}_{8} \mathrm{H}_{7} \mathrm{BrO}_{3}$ requires $\mathrm{C}$, 41.6; H, 3.05\%); $v_{\max } / \mathrm{cm}^{-1} 3071(\mathrm{O}-\mathrm{H}), 3006,2944,2901$ and $2840(\mathrm{C}-\mathrm{H}), 1644(\mathrm{C}=\mathrm{O}), 1609$ and $1582(\mathrm{C}=\mathrm{C}), 1315,1237,1129$ and $1036(\mathrm{C}-\mathrm{O}) ; \delta_{H}\left(300 \mathrm{MHz}, \mathrm{d}_{6}-\mathrm{DMSO}\right) 3.78(3 \mathrm{H}, \mathrm{s}$, $\left.\mathrm{OCH}_{3}\right), 7.31(1 \mathrm{H}, \mathrm{d}, J=3.0 \mathrm{~Hz}, 6-\mathrm{H}), 7.51(1 \mathrm{H}, \mathrm{d}, J=3.0 \mathrm{~Hz}, 4-\mathrm{H}), 10.08(1 \mathrm{H}, \mathrm{s}, \mathrm{CHO}), 10.65$ $(1 \mathrm{H}, \mathrm{s}, \mathrm{OH}) ; \delta_{C}\left(75 \mathrm{MHz}, \mathrm{d}_{6}\right.$-DMSO) $56.5\left(\mathrm{OCH}_{3}, 8-\mathrm{C}\right), 112.4$ (quat., 3-C), 114.7 (CH, 6-C), 123.3 (quat., 1-C), 126.6 (CH, 4-C), 151.4 (quat., 2-C), 153.1 (quat., 5-C), 194.5 (CHO, 7-C). 


\subsubsection{3-Bromo-2,5-dimethoxybenzaldehyde 28}<smiles>COc1cc(Br)c(OC)c(C=O)c1</smiles>

An oven dried round bottomed flask was charged with 3-bromo-2-hydroxy-5methoxybenzaldehyde $27(12.86 \mathrm{~g}, 55.70 \mathrm{mmol}), \mathrm{K}_{2} \mathrm{CO}_{3}(10.78 \mathrm{~g}, 77.98 \mathrm{mmol})$ and dry DMF $(300 \mathrm{~mL})$. The resulting mixture was stirred for $5 \mathrm{~min}$ and DMS $(7.38 \mathrm{~mL}, 77.98 \mathrm{mmol})$ was then added dropwise. The reaction was left stirring overnight at ambient temperature. The resulting olive green coloured mixture was quenched with water resulting in the formation of a white precipitate. This mixture was extracted with $\mathrm{Et}_{2} \mathrm{O}(4 \times 300 \mathrm{~mL})$ and the combined organic layers were washed with brine $(3 \times 250 \mathrm{~mL})$ and dried $\left(\mathrm{MgSO}_{4}\right)$. The solvent was removed in vacuo yielding 3-bromo-2,5-dimethoxybenzaldehyde 28 as a white crystalline solid (13.61g, 55.54mmol, 99.7\%). White crystals were obtained from petroleum ether (60$80^{\circ} \mathrm{C}$ ); mp $62.5-63.5^{\circ} \mathrm{C}$ [lit. $\mathrm{mp} 68^{\circ} \mathrm{C}^{3}$ ]; (found: $\mathrm{C}, 41.1$; $\mathrm{H}, 3.6 \% . \mathrm{C}_{9} \mathrm{H}_{9} \mathrm{BrO}_{3}$ requires $\mathrm{C}, 41.1$; $\mathrm{H}, 3.7 \%) ; v_{\max } / \mathrm{cm}^{-1} 2935,2854$ and $2832(\mathrm{C}-\mathrm{H}), 1685(\mathrm{C}=\mathrm{O}), 1600(\mathrm{C}=\mathrm{C}), 1225,1205$ and $1042(\mathrm{C}-\mathrm{O}) ; \delta_{H}\left(300 \mathrm{MHz}, \mathrm{CDCl}_{3}\right) 3.85\left(3 \mathrm{H}, \mathrm{s}, \mathrm{OCH}_{3}, 8-\mathrm{H}\right), 3.97\left(3 \mathrm{H}, \mathrm{s}, \mathrm{OCH}_{3}, 9-\mathrm{H}\right), 7.30$ $(1 \mathrm{H}, \mathrm{d}, J=3.0 \mathrm{~Hz}, 6-\mathrm{H}), 7.41(1 \mathrm{H}, \mathrm{d}, J=3.0 \mathrm{~Hz}, 4-\mathrm{H}), 10.34(1 \mathrm{H}, \mathrm{s}, \mathrm{CHO}) ; \delta_{C}(75 \mathrm{MHz}$, $\left.\mathrm{CDCl}_{3}\right) 56.0\left(\mathrm{OCH}_{3}, 8-\mathrm{C}\right), 63.8\left(\mathrm{OCH}_{3}, 9-\mathrm{C}\right), 110.3(\mathrm{CH}, 6-\mathrm{C}), 118.6$ (quat., 3-C), $126.5(\mathrm{CH}$, 4-C), 130.6 (quat., 1-C), 154.4 (quat., 5-C), 156.5 (quat., 2-C), 189.0 (CHO, 7-C).

\subsubsection{2,5-Dimethoxybiphenyl-3-carbaldehyde $3 f$}

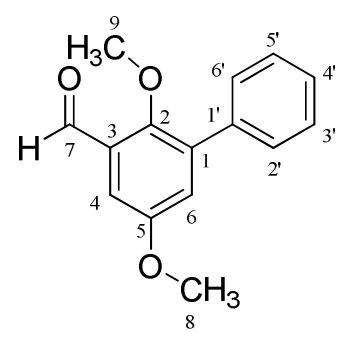

A two necked round bottomed flask equipped with a reflux condenser was charged with 3bromo-2,5-dimethoxybenzaldehyde 28 (2.72g, 11.10mmol), phenylboronic acid (1.62g, $13.29 \mathrm{mmol})$ and DME $(75 \mathrm{~mL})$. The resulting solution was degassed with $\mathrm{N}_{2}$, with stirring, over $15 \mathrm{~min}$. A separate round bottomed flask was charged with $\mathrm{Na}_{2} \mathrm{CO}_{3}(2 \mathrm{M}, 20 \mathrm{~mL})$ and EtOH $(20 \mathrm{~mL})$; the resulting mixture was also degassed with $\mathrm{N}_{2}$ over $15 \mathrm{~min}$. It was then transferred, along with $\mathrm{Pd}\left(\mathrm{Ph}_{3}\right)_{4}(0.64 \mathrm{~g}, 0.56 \mathrm{mmol})$, into the two necked round bottomed flask 
containing the 3-bromo-2,5-dimethoxybenzaldehyde $\mathbf{2 8}$ solution. The resulting mixture was brought to reflux $\left(\mathrm{T}_{\text {oil bath }}=100^{\circ} \mathrm{C}\right)$, while keeping a constant $\mathrm{N}_{2}$ flow over the reaction. The reflux was stopped after $23 \mathrm{~h}$ and the reaction cooled to $\mathrm{rt}$, then the reaction was cooled with an ice bath and acidified (to $\mathrm{pH} 1-2)$ with $\mathrm{HCl}(10 \%)$. The resulting mixture was diluted with EtOAc and filtered through Celite, which was washed several times with small portions of EtOAc. The filtrate was decanted into a separating funnel and the aqueous layer was further extracted with EtOAc $(3 \times 50 \mathrm{~mL})$. The combined organic layers were washed with water $(100 \mathrm{~mL})$ and brine $(100 \mathrm{~mL})$, and dried $\left(\mathrm{MgSO}_{4}\right)$. The solvent was evaporated in vacuo and the residue subjected to column chromatography, eluting with petroleum ether $\left(60-80^{\circ} \mathrm{C}\right)$ : $\mathrm{Et}_{2} \mathrm{O}$ (80:20). 2,5-Dimethoxybiphenyl-3-carbaldehyde 3f was isolated as clear cubic white plates $(2.29 \mathrm{~g}, 9.43 \mathrm{mmol}, 85.0 \%)$; $\mathrm{mp}\left(\mathrm{Et}_{2} \mathrm{O}\right.$ : petroleum ether $\left.60-80^{\circ} \mathrm{C}\right) 64.5-65.5^{\circ} \mathrm{C}$; (found: $\mathrm{C}, 74.3 ; \mathrm{H}, 5.9 \% . \mathrm{C}_{15} \mathrm{H}_{14} \mathrm{O}_{3}$ requires $\left.\mathrm{C}, 74.4 ; \mathrm{H}, 5.8 \%\right) ; \mathrm{m} / \mathrm{Z} 243.0(M H)^{+}, 265.0(M N a)^{+}$; $v_{\max } / \mathrm{cm}^{-1} 2877$ and $2754(\mathrm{C}-\mathrm{H}), 1680(\mathrm{C}=\mathrm{O}), 1596(\mathrm{C}=\mathrm{C}), 1229$ and $1041(\mathrm{C}-\mathrm{O}) ; \delta_{H}(300$ $\left.\mathrm{MHz}, \mathrm{CDCl}_{3}\right) 3.51\left(3 \mathrm{H}, \mathrm{s}, \mathrm{OCH}_{3}, 9-\mathrm{H}\right), 3.89\left(3 \mathrm{H}, \mathrm{s}, \mathrm{OCH}_{3}, 8-\mathrm{H}\right), 7.22(1 \mathrm{H}, \mathrm{d}, J=3.3 \mathrm{~Hz}, 6-$ H), $7.36(1 \mathrm{H}, \mathrm{d}, J=3.3 \mathrm{~Hz}, 4-\mathrm{H}), 7.41-7.53$ (3H, m, 3'-H, 4'-H and 5'-H), 7.60-7.64 (2H, m, 2'$\mathrm{H}$ and 6'-H), $10.48(1 \mathrm{H}, \mathrm{CHO}, 7-\mathrm{H}) ; \delta_{C}\left(75 \mathrm{MHz}, \mathrm{CDCl}_{3}\right) 55.8\left(\mathrm{OCH}_{3}, 8-\mathrm{C}\right), 62.9\left(\mathrm{OCH}_{3}, 9-\right.$ C), 109.1 (CH, 4-C), 124.75 (CH, 6-C), 127.9 (CH, 4'-C), 128.6 (CH, 3'-C and 5'-C), 128.9 (CH, 2'-C and 6'-C), 130.1 (quat., 3-C), 136.9 (quat., 1-C), 137.3 (quat., 1'-C), 155.4 (quat., 2C), 156.0 (quat., 5-C), 190.05 (CHO, 7-C).

\subsubsection{General procedure for the Baeyer-Villiger oxidation of benzaldehydes $3 \mathrm{e}$ and $3 \mathrm{f}$}

In a two necked round bottomed flask equipped with a reflux condenser, the correctly substituted benzaldehyde ( 1 mol equivalent) was dissolved in DCM $(70 \mathrm{~mL})$. The resulting solution was cooled in an ice bath and meta-chloroperbenzoic acid ( 1.7 mol equivalent) was added portion-wise. The ice bath was removed $1 \mathrm{~h}$ after the end of the addition and the resulting solution left with stirring overnight at $\mathrm{rt}$. The reaction progress was monitored by TLC and the reaction was refluxed for $5-6 \mathrm{~h}$ to drive it to completion, when necessary. The reaction, after cooling to $\mathrm{rt}$ if heated, was washed with $\mathrm{NaHCO}_{3}(5 \%, 3 \times 100 \mathrm{~mL})$. The DCM layer was then dried $\left(\mathrm{MgSO}_{4}\right)$ and the solvent removed in vacuo. The residual oil was dissolved in methanol $(20 \mathrm{~mL})$ and $\mathrm{NaOH}(10 \%$, excess) was added; the resulting dark solution was stirred for 2-3 hours and then acidified (to $\mathrm{pH} \mathrm{1-2)} \mathrm{with} \mathrm{HCl}(10 \%)$. The resulting mixture was extracted with DCM $(3 \times 80 \mathrm{~mL})$ and the combined organic extracts were successively washed with $\mathrm{NaHCO}_{3}(5 \%, 100 \mathrm{~mL})$, water $(100 \mathrm{~mL})$ and brine $(100 \mathrm{~mL})$, 
and dried $\left(\mathrm{MgSO}_{4}\right)$. The solvent was removed in vacuo and the residue subjected to column chromatography on silica gel. The product was recrystallized from petroleum ether $\left(60-80^{\circ} \mathrm{C}\right)$.

\subsubsection{2,5-Dimethoxy-4-pentylphenol 4e}

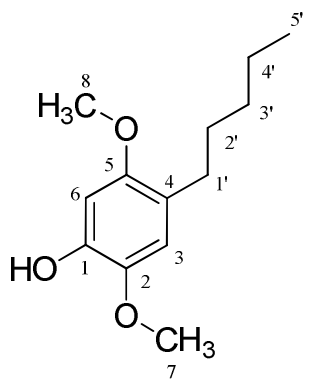

Prepared from 2,5-dimethoxy-4-pentylbenzaldehyde 3e $(3.44 \mathrm{~g}, 14.58 \mathrm{mmol})$; elution with petroleum ether $\left(60-80^{\circ} \mathrm{C}\right): \mathrm{Et}_{2} \mathrm{O}(70: 30)$ yielded 2,5-dimethoxy-4-pentylphenol $4 \mathbf{e}$ as a white crystalline solid $(2.66 \mathrm{~g}, 11.87 \mathrm{mmol}, 81.4 \%)$. Recrystallization from $\mathrm{Et}_{2} \mathrm{O}$ : petroleum ether $\left(60-80^{\circ} \mathrm{C}\right.$ ) produced white needles, mp 33-35 ${ }^{\circ} \mathrm{C}$; (found: $\mathrm{C}, 69.55 ; \mathrm{H}, 9.0 \% . \mathrm{C}_{13} \mathrm{H}_{20} \mathrm{O}_{3}$ requires $\mathrm{C}, 69.6 ; \mathrm{H}, 9.0 \%) ; \mathrm{m} / \mathrm{Z} 225.0(\mathrm{MH})^{+} ; v_{\max } / \mathrm{cm}^{-1} 3354(\mathrm{O}-\mathrm{H}), 2953$ and $2930(\mathrm{C}-\mathrm{H})$, 1598 and $1515(\mathrm{C}=\mathrm{C}), 1192$ and $1038(\mathrm{C}-\mathrm{O}) ; \delta_{H}\left(300 \mathrm{MHz}, \mathrm{CDCl}_{3}\right) 0.96(3 \mathrm{H}, \mathrm{t}, J=6.9 \mathrm{~Hz}$, 5'-H), 1.38-1.415 (4H, m, 3'-H and 4'-H), 1.58-1.63 (2H, m, 2'-H), 2.59 (2H, t, $J=7.5 \mathrm{~Hz}, 1^{\prime}-$ H), $3.81\left(3 \mathrm{H}, \mathrm{s}, \mathrm{OCH}_{3}, 8-\mathrm{H}\right), 3.89\left(3 \mathrm{H}, \mathrm{s}, \mathrm{OCH}_{3}, 7-\mathrm{H}\right), 5.61(1 \mathrm{H}, \mathrm{s}, \mathrm{OH}), 6.60(1 \mathrm{H}, \mathrm{s}, 6-\mathrm{H})$, $6.73(1 \mathrm{H}, \mathrm{s}, 3-\mathrm{H}) ; \delta_{C}\left(75 \mathrm{MHz}, \mathrm{CDCl}_{3}\right) 14.1\left(\mathrm{CH}_{3}, 5^{\prime}-\mathrm{C}\right), 22.6\left(\mathrm{CH}_{2}, 3^{\prime}-\mathrm{C}\right.$ or 4'-C), $29.8\left(\mathrm{CH}_{2}\right.$, 1'-C), $30.1\left(\mathrm{CH}_{2}, 2^{\prime}-\mathrm{C}\right), 31.8\left(\mathrm{CH}_{2}, 3^{\prime}-\mathrm{C}\right.$ or 4'-C), $56.1\left(\mathrm{OCH}_{3}, 8-\mathrm{C}\right), 56.8\left(\mathrm{OCH}_{3}, 7-\mathrm{C}\right), 99.5$ (CH, 6-C), 113.3 (CH, 3-C), 122.3 (quat., 4-C), 140.1 (quat., 2-C), 144.1 (quat., 1-C), 152.0 (quat., 5-C).

\subsubsection{2,5-Dimethoxybiphenyl-3-ol 4f}

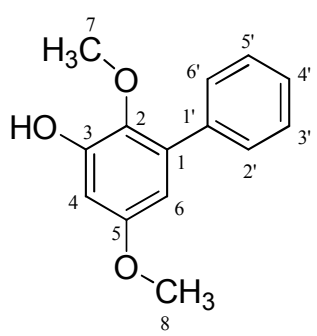

The title compound was prepared as described from 2,5-dimethoxybiphenyl-3-carbaldehyde 3f $(2.02 \mathrm{~g}, 8.34 \mathrm{mmol})$; elution with petroleum ether $\left(60-80^{\circ} \mathrm{C}\right): \mathrm{Et}_{2} \mathrm{O}$ (70:30) yielded 2,5dimethoxybiphenyl-3-ol $\mathbf{4 f}$ as a white crystalline solid (1.43g, 6.19mmol, 74.2\%). White needles were obtained from petroleum ether $\left(60-80^{\circ} \mathrm{C}\right) ; \mathrm{mp} 67-69^{\circ} \mathrm{C}$; (found: $\mathrm{C}, 73.1 ; \mathrm{H}$, 
6.2\%. $\mathrm{C}_{14} \mathrm{H}_{14} \mathrm{O}_{3}$ requires C, 73.0; H, 6.1\%); $\mathrm{m} / \mathrm{Z} 231.0(M H)^{+} ; v_{\max } / \mathrm{cm}^{-1} 3396(\mathrm{O}-\mathrm{H}), 1619$, 1591 and $1573(\mathrm{C}=\mathrm{C}), 1206$ and $1049(\mathrm{C}-\mathrm{O}) ; \delta_{H}\left(300 \mathrm{MHz}, \mathrm{CDCl}_{3}\right) 3.44\left(3 \mathrm{H}, \mathrm{s}, \mathrm{OCH}_{3}, 7-\mathrm{H}\right)$, $3.85\left(3 \mathrm{H}, \mathrm{s}, \mathrm{OCH}_{3}, 8-\mathrm{H}\right), 6.10(1 \mathrm{H}, \mathrm{s}, \mathrm{OH}), 6.51(1 \mathrm{H}, \mathrm{d}, J=3.0 \mathrm{~Hz}, 6-\mathrm{H}), 6.64(1 \mathrm{H}, \mathrm{d}, J=$ 3.0Hz, 4-H), 7.39-7.52 (3H, m, 3'-H, 5'-H and 4'-H), 7.64-7.67 (2H, m, 2'-H and 6'-H); $\delta_{C}(75$ $\left.\mathrm{MHz}, \mathrm{CDCl}_{3}\right) 55.6\left(\mathrm{OCH}_{3}, 8-\mathrm{C}\right), 60.9\left(\mathrm{OCH}_{3}, 7-\mathrm{C}\right), 100.6(\mathrm{CH}, 4-\mathrm{C}), 107.2(\mathrm{CH}, 6-\mathrm{C}), 127.6$ $\left(\mathrm{CH}, 4{ }^{\prime}-\mathrm{C}\right), 128.5\left(\mathrm{CH}, 3^{\prime}-\mathrm{C}\right.$ and 5'-C), $128.8\left(\mathrm{CH}, 2^{\prime}-\mathrm{C}\right.$ and 6'-C), 134.7 (quat., 1-C), 138.0 (quat., 1'-C), 138.5 (quat., 2-C), 149.9 (quat., 3-C), 156.45 (quat., 5-C).

\subsection{Synthesis of diphenylethers 6a-f}

\subsubsection{General procedure}

The appropriately substituted phenol (1 mol equivalent) was dissolved in DMSO $(5 \mathrm{~mL} / \mathrm{mmol}$ of substituted phenol) and $\mathrm{Et}_{3} \mathrm{~N}$ (1mol equivalent) was added immediately. The resulting solution was stirred for $5 \mathrm{~min}$, before 2,4-dinitro-1-fluorobenzene (1mol equivalent) was added; the solution became an orange-red colour. The reaction was left stirring for 5 hours, then quenched with water $(5 \mathrm{~mL} / \mathrm{mL}$ of DMSO) and the resulting mixture extracted with DCM $(3 \times 0.5 \mathrm{~mL} / \mathrm{mL}$ of suspension). The combined DCM extracts were successively washed with $\mathrm{NaOH}(10 \%, 3 \times 0.5 \mathrm{~mL} / \mathrm{mL}$ of DCM extract), water $(0.5 \mathrm{~mL} / \mathrm{mL}$ of DCM extract), brine $\left(0.5 \mathrm{~mL} / \mathrm{mL}\right.$ of DCM extract), and dried $\left(\mathrm{MgSO}_{4}\right)$. The solvent was removed in vacuo and the residue was either subjected to column chromatography or directly recrystallised from ethanol.

\subsubsection{2,4-Dinitro-2',5'-dimethoxydiphenylether 6a}

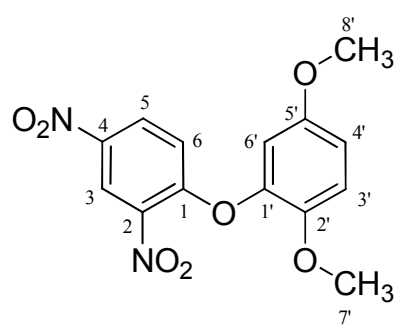

Prepared from 2,5-dimethoxyphenol 4a $(6.38 \mathrm{~g}, 41.37 \mathrm{mmol})$ and 2,4-dinitrofluorobenzene 5 (7.70g, 41.37mmol). 2,4-Dinitro-2,5-dimethoxydiphenylether 6a (11.68g, 36.47mmol, $88.2 \%$ ) was isolated as crescent shaped yellow crystals from EtOH; mp $95-96^{\circ} \mathrm{C} ; \mathrm{m} / \mathrm{Z} 343.1$ $(M N a)^{+}$; (found C, 52.4; H, 3.7; N, 8.7\%. $\mathrm{C}_{14} \mathrm{H}_{12} \mathrm{~N}_{2} \mathrm{O}_{6}$ requires C, 52.5; H, 3.8; N, 8.75\%); $v_{\max } / \mathrm{cm}^{-1} 1604(\mathrm{C}=\mathrm{C}), 1506$ and $1340\left(\mathrm{NO}_{2}\right), 1274,1035$ and $1014(\mathrm{C}-\mathrm{O}) ; \delta_{H}\left(300 \mathrm{MHz}, \mathrm{d}_{6^{-}}\right.$ DMSO 3.69 (3H, s, $\left.\mathrm{OCH}_{3}, 7^{\prime}-\mathrm{H}\right), 3.74\left(3 \mathrm{H}, \mathrm{s}, \mathrm{OCH}_{3}, 8^{\prime}-\mathrm{H}\right), 6.92\left(1 \mathrm{H}, \mathrm{dd}, J=9.0,3.0 \mathrm{~Hz}, 4^{\prime}-\right.$ 
H), $6.98\left(1 \mathrm{H}, \mathrm{d}, J=2.7 \mathrm{~Hz}, 6{ }^{\prime}-\mathrm{H}\right), 6.99(1 \mathrm{H}, \mathrm{d}, J=9.3 \mathrm{~Hz}, 6-\mathrm{H}), 7.20\left(1 \mathrm{H}, \mathrm{d}, J=9.0 \mathrm{~Hz}, 3^{\prime}-\mathrm{H}\right)$, $8.39(1 \mathrm{H}, \mathrm{dd}, J=9.3,3.0 \mathrm{~Hz}, 5-\mathrm{H}), 8.86(1 \mathrm{H}, \mathrm{d}, J=3.0 \mathrm{~Hz}, 3-\mathrm{H}) ; \delta_{C}\left(75 \mathrm{MHz}, \mathrm{d}_{6}-\mathrm{DMSO}\right) 56.2$ $\left(\mathrm{OCH}_{3}, 7^{\prime}-\mathrm{C}\right), 56.8\left(\mathrm{OCH}_{3}, 8^{\prime}-\mathrm{C}\right), 109.1\left(\mathrm{CH}, 6{ }^{\prime}-\mathrm{C}\right), 113.1\left(\mathrm{CH}, 4^{\prime}-\mathrm{C}\right), 115.3\left(\mathrm{CH}, 3^{\prime}-\mathrm{C}\right), 117.9$ (CH, 6-C), 122.2 (CH, 3-C), 129.9 (CH, 5-C), 138.6 (quat., 2-C), 141.4 (quat., 4-C), 141.7 (quat., 1'-C), 145.1 (quat., 2'-C), 154.3 (quat., 5'-C), 155.6 (quat., 1-C).

\subsubsection{2,4-Dinitro-2',5'-dimethoxy-3',4'-dimethyldiphenylether $6 \mathrm{~b}$}

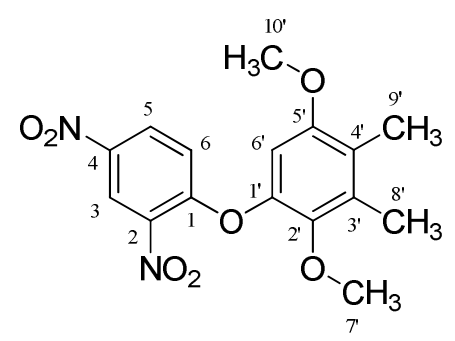

Prepared from 2,5-dimethoxy-3,4-dimethylphenol $4 \mathbf{b}(0.65 \mathrm{~g}, 3.56 \mathrm{mmol})$. Elution with petroleum ether $\left(60-80^{\circ} \mathrm{C}\right)$ : EtOAc (70:30) yielded 2,4-dinitro-2',5'-dimethoxy-3',4'dimethyldiphenylether $\mathbf{6 b}$ as an orange solid $(0.76 \mathrm{~g}, 2.19 \mathrm{mmol}, 61.6 \%)$. Orange crystals were obtained from aqueous ethanol, mp $100^{\circ} \mathrm{C}$; (found $\mathrm{C}, 55.4 ; \mathrm{H}, 4.6 ; \mathrm{N}, 8.0 \% . \mathrm{C}_{16} \mathrm{H}_{16} \mathrm{~N}_{2} \mathrm{O}_{7}$ requires $\mathrm{C}, 55.2 ; \mathrm{H}, 4.6 ; \mathrm{N}, 8.0 \%) ; \mathrm{m} / \mathrm{z} 371.0(\mathrm{MNa})^{+} ; v_{\max } / \mathrm{cm}^{-1} 1606(\mathrm{C}=\mathrm{C}), 1529$ and 1345 $\left(\mathrm{NO}_{2}\right), 1263,1233$ and $1026(\mathrm{C}-\mathrm{O}) ; \delta_{H}\left(300 \mathrm{MHz}, \mathrm{d}_{6}-\mathrm{DMSO}\right) 2.13\left(3 \mathrm{H}, \mathrm{s}, \mathrm{CH}_{3}, 9^{\prime}-\mathrm{H}\right), 2.19$ $\left(3 \mathrm{H}, \mathrm{s}, \mathrm{CH}_{3}, 8^{\prime}-\mathrm{H}\right), 3.60\left(3 \mathrm{H}, \mathrm{s}, \mathrm{OCH}_{3}, 7^{\prime}-\mathrm{H}\right), 3.77$ (3H, s, OCH, $\left.10^{\prime}-\mathrm{H}\right), 6.89\left(1 \mathrm{H}, \mathrm{s}, 66^{\prime}-\mathrm{H}\right)$, $7.01(1 \mathrm{H}, \mathrm{d}, J=9.0 \mathrm{~Hz}, 6-\mathrm{H}), 8.38(1 \mathrm{H}, \mathrm{dd}, J=9.3,3.0 \mathrm{~Hz}, 5-\mathrm{H}), 8.88(1 \mathrm{H}, \mathrm{d}, J=3.0 \mathrm{~Hz}, 3-\mathrm{H})$; $\delta_{C}\left(75 \mathrm{MHz}, \mathrm{d}_{6}\right.$-DMSO) $12.3\left(\mathrm{CH}_{3}, 9^{\prime}-\mathrm{C}\right), 12.8\left(\mathrm{CH}_{3}, 8^{\prime}-\mathrm{C}\right), 56.5\left(\mathrm{OCH}_{3}, 10^{\prime}-\mathrm{C}\right), 61.4\left(\mathrm{OCH}_{3}\right.$, 7'-C), 103.4 (CH, 6'-C), 118.0 (CH, 6-C), 122.1 (CH, 3-C), 124.4 (quat., 4'-C), 129.9 (CH, 5C), 132.9 (quat., 3'-C), 138.7 (quat., 2-C), 141.3 (quat., 4-C), 143.2 (quat., 1'-C), 143.7 (quat., 2'-C), 154.4 (quat., 5'-C), 155.7 (quat., 1-C).

\subsubsection{2,4-Dinitro-2',5'-dimethoxy-3',4',6'-trimethyldiphenylether 6c}

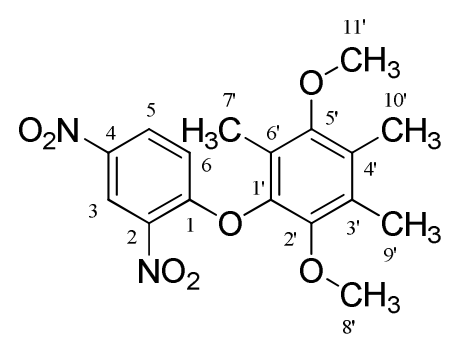

Prepared from 2,5-dimethoxy-3,4,6-trimethylphenol 4c $(0.37 \mathrm{~g}, 1.89 \mathrm{mmol})$, using 1,8diazabicyclo[5-4-0]undec-7-ene (DBU) $(0.30 \mathrm{~mL}, 2.00 \mathrm{mmol})$ instead of $\mathrm{Et}_{3} \mathrm{~N}$. Elution with 
petroleum ether $\left(60-80^{\circ} \mathrm{C}\right)$ : EtOAc (30:70) yielded 2,4-dinitro-2',5'-dimethoxy-3',4',6'trimethyldiphenylether $\mathbf{6 c}$ as an off-white solid $(0.59 \mathrm{~g}, 1.63 \mathrm{mmol}, 85.7 \%)$. Long off-white needles were obtained from ethanol; $\mathrm{mp} 142^{\circ} \mathrm{C}$; (found $\mathrm{C}, 56.4 ; \mathrm{H}, 5.05 ; \mathrm{N}, 7.7 \%$. $\mathrm{C}_{17} \mathrm{H}_{18} \mathrm{~N}_{2} \mathrm{O}_{7}$ requires $\left.\mathrm{C}, 56.35 ; \mathrm{H}, 5.0 ; \mathrm{N}, 7.7 \%\right) ; \mathrm{m} / \mathrm{Z} 385.1(M N a)^{+} ; v_{\max } / \mathrm{cm}^{-1} 1609(\mathrm{C}=\mathrm{C})$, 1540 and $1345\left(\mathrm{NO}_{2}\right), 1260,1250$ and $1072(\mathrm{C}-\mathrm{O}) ; \delta_{H}\left(300 \mathrm{MHz}, \mathrm{CDCl}_{3}\right) 2.04\left(3 \mathrm{H}, \mathrm{s}, \mathrm{CH}_{3}\right.$, 7'-H), 2.12 (3H, s, $\left.\mathrm{CH}_{3}, 10^{\prime}-\mathrm{H}\right), 2.16$ (3H, s, $\left.\mathrm{CH}_{3}, 9^{\prime}-\mathrm{H}\right), 3.59$ (3H, s, $\left.\mathrm{OCH}_{3}, 8^{\prime}-\mathrm{H}\right), 3.63$ (3H, s, $\left.\mathrm{OCH}_{3}, 11^{\prime}-\mathrm{H}\right), 6.69(1 \mathrm{H}, \mathrm{d}, J=9.3 \mathrm{~Hz}, 6-\mathrm{H}), 8.15(1 \mathrm{H}, \mathrm{dd}, J=9.3,2.7 \mathrm{~Hz}, 5-\mathrm{H}), 8.76(1 \mathrm{H}, \mathrm{d}, J$ $=2.7 \mathrm{~Hz}, 3-\mathrm{H}) ; \delta_{C}\left(75 \mathrm{MHz}, \mathrm{CDCl}_{3}\right) 9.6\left(\mathrm{CH}_{3}, 7^{\prime}-\mathrm{C}\right), 12.4\left(\mathrm{CH}_{3}, 9^{\prime}-\mathrm{C}\right.$ or $\left.10^{\prime}-\mathrm{C}\right), 12.8\left(\mathrm{CH}_{3}, 9^{\prime}-\right.$ $\mathrm{C}$ or 10'-C), $60.5\left(\mathrm{OCH}_{3}, 8{ }^{\prime}-\mathrm{C}\right), 61.2\left(\mathrm{OCH}_{3}, 11^{\prime}-\mathrm{C}\right), 116.6(\mathrm{CH}, 6-\mathrm{C}), 122.0(\mathrm{CH}, 3-\mathrm{C}), 122.1$ (quat., 6'-C), 128.9 (CH, 5-C), 129.6 (quat., 3'-C or 4'-C), 130.0 (quat., 3'-C or 4'-C), 138.15 (quat., 2-C), 141.0 (quat., 4-C), 142.2 (quat., 1'-C), 146.1 (quat., 2'-C), 153.5 (quat., 5'-C), 155.8 (quat., 1-C).

\subsubsection{2,4-Dinitro-2',5'-dimethoxy-4'-'butyldiphenylether 6d}

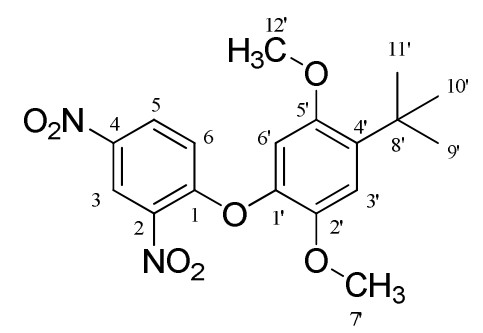

Prepared from 2,5-dimethoxy-4- ${ }^{t}$ butylphenol 4d (3.55g, 16.88mmol). Elution with petroleum ether $\left(60-80^{\circ} \mathrm{C}\right): \mathrm{Et}_{2} \mathrm{O}(70: 30)$ yielded 2,4-dinitro-2',5'-dimethoxy-4'-'butyldiphenylether $\mathbf{6 d}$ as a yellow crystalline solid $(6.06 \mathrm{~g}, 16.09 \mathrm{mmol}, 95.8 \%)$. Recrystallization from EtOH produced small bright yellow needles; mp $131-132^{\circ} \mathrm{C}$; (found $\mathrm{C}, 57.45 ; \mathrm{H}, 5.4 ; \mathrm{N}, 7.4 \%$. $\mathrm{C}_{18} \mathrm{H}_{20} \mathrm{~N}_{2} \mathrm{O}_{7}$ requires C, 57.4; H, 5.4; N, 7.4\%); $m / z 399.1(M N a)^{+} ; v_{\max } / \mathrm{cm}^{-1} 1608(\mathrm{C}=\mathrm{C})$, 1535 and $1342\left(\mathrm{NO}_{2}\right), 1209$ and $1031(\mathrm{C}-\mathrm{O}) ; \delta_{H}\left(300 \mathrm{MHz}, \mathrm{CDCl}_{3}\right) 1.32\left(9 \mathrm{H}, \mathrm{s}, \mathrm{C}\left(\mathrm{CH}_{3}\right)_{3}\right)$, $3.64\left(3 \mathrm{H}, \mathrm{s}, \mathrm{OCH}_{3}, 7^{\prime}-\mathrm{H}\right), 3.72\left(3 \mathrm{H}, \mathrm{s}, \mathrm{OCH}_{3}, 12^{\prime}-\mathrm{H}\right), 6.66\left(1 \mathrm{H}, \mathrm{s}, 66^{\prime}-\mathrm{H}\right), 6.84(1 \mathrm{H}, \mathrm{d}, J=$ 9.3Hz, 6-H), $6.94(1 \mathrm{H}, \mathrm{s}, 3 \mathrm{\prime}-\mathrm{H}), 8.17(1 \mathrm{H}, \mathrm{dd}, J=9.3,3.0 \mathrm{~Hz}, 5-\mathrm{H}), 8.74(1 \mathrm{H}, \mathrm{d}, J=2.7 \mathrm{~Hz}, 3-$ $\mathrm{H}) ; \delta_{C}\left(75 \mathrm{MHz}, \mathrm{CDCl}_{3}\right) 29.7\left(3 \times \mathrm{CH}_{3}, 9^{\prime} / 10^{\prime} / 11^{\prime}-\mathrm{C}\left(\mathrm{CH}_{3}\right)_{3}\right), 35.2$ (quat., 8'-C( $\left.\left.\mathrm{CH}_{3}\right)_{3}\right), 55.8$ $\left(\mathrm{OCH}_{3}, 7^{\prime}-\mathrm{C}\right), 57.1\left(\mathrm{OCH}_{3}, 2^{\prime}-\mathrm{C}\right), 106.5\left(\mathrm{CH}, 6{ }^{\prime}-\mathrm{C}\right), 113.8\left(\mathrm{CH}, 3{ }^{\prime}-\mathrm{C}\right), 117.5(\mathrm{CH}, 6-\mathrm{C})$, $122.0(\mathrm{CH}, 3-\mathrm{C}), 128.6$ (CH, 5-C), 138.1 (quat., 4'-C), 138.3 (quat., 2-C), 139.5 (quat., 1'-C), 141.0 (quat., 4-C), 143.8 (quat., 2'-C), 153.4 (quat., 5'-C), 156.5 (quat., 1-C). 


\subsubsection{2,4-Dinitro-2',5'-dimethoxy-4'-pentyldiphenylether 6 e}

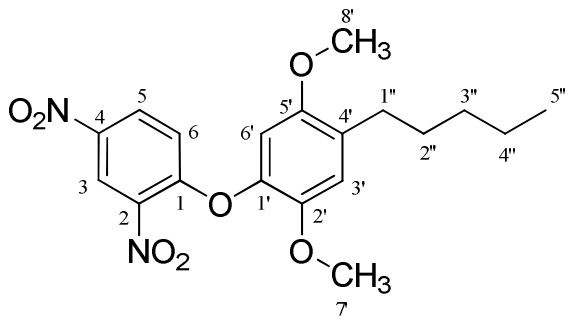

Prepared from 2,5-dimethoxy-4-pentylphenol 4e (2.71g, 12.09mmol). 2,4-Dinitro-2',5'dimethoxy-4'-pentyldiphenylether $\mathbf{6 e}$ was isolated as a yellow crystalline solid (4.36g, 11.17mmol, 92.4\%). Recrystallization from EtOH yielded small yellow needles; mp (EtOH): $85-87^{\circ} \mathrm{C}$; (found: $\mathrm{C}, 58.3 ; \mathrm{H}, 5.7 ; \mathrm{N}, 7.2 \% . \mathrm{C}_{19} \mathrm{H}_{22} \mathrm{~N}_{2} \mathrm{O}_{7}$ requires $\mathrm{C}, 58.5 ; \mathrm{H}, 5.7 ; \mathrm{N}, 7.2 \%$ ); $m / Z 390.1(M H)^{+}, 413.1(M N a)^{+} ; v_{\max } / \mathrm{cm}^{-1} 2855-2957(\mathrm{C}-\mathrm{H}), 1608,1508(\mathrm{C}=\mathrm{C}), 1536$ and $1342\left(\mathrm{NO}_{2}\right), 1212$ and $1037(\mathrm{C}-\mathrm{O}) ; \delta_{H}\left(300 \mathrm{MHz}, \mathrm{CDCl}_{3}\right) 0.96(3 \mathrm{H}, \mathrm{t}, J=6.9 \mathrm{~Hz}, 5 "-\mathrm{H}), 1.39$ 1.43 (4H, m, 3"-H and 4"-H), 1.62-1.67 (2H, m, 2"-H), 2.66 (2H, t, $J=7.5 \mathrm{~Hz}, 1 "-\mathrm{H}), 3.755$ $\left(3 \mathrm{H}, \mathrm{s}, \mathrm{OCH}_{3}, 7^{\prime}-\mathrm{H}\right), 3.82\left(3 \mathrm{H}, \mathrm{s}, \mathrm{OCH}_{3}, 8^{\prime}-\mathrm{H}\right), 6.76\left(1 \mathrm{H}, \mathrm{s}, 6^{\prime}-\mathrm{H}\right), 6.91\left(1 \mathrm{H}, \mathrm{s}, 3{ }^{\prime}-\mathrm{H}\right), 6.96(1 \mathrm{H}$, d, $J=9.3 \mathrm{~Hz}, 6-\mathrm{H}), 8.31(1 \mathrm{H}, \mathrm{dd}, J=9.3,2.7 \mathrm{~Hz}, 5-\mathrm{H}), 8.87(1 \mathrm{H}, \mathrm{d}, J=3.0 \mathrm{~Hz}, 3-\mathrm{H}) ; \delta_{C}(75$ $\left.\mathrm{MHz}, \mathrm{CDCl}_{3}\right) 14.1\left(\mathrm{CH}_{3}, 5 "-\mathrm{C}\right), 22.6\left(\mathrm{CH}_{2}, 3\right.$ "-C or 4"-C), $29.6\left(\mathrm{CH}_{2}, 2 "-\mathrm{C}\right), 30.1\left(\mathrm{CH}_{2}, 1 "-\mathrm{C}\right)$, $31.75\left(\mathrm{CH}_{2}, 3\right.$ "-C or 4"-C), $56.1\left(\mathrm{OCH}_{3}, 8^{\prime}-\mathrm{C}\right), 56.9\left(\mathrm{OCH}_{3}, 7^{\prime}-\mathrm{C}\right), 105.6\left(\mathrm{CH}, 6{ }^{\prime}-\mathrm{C}\right), 115.85$ (CH, 3'-C), $117.4(\mathrm{CH}, 6-\mathrm{C}), 122.0(\mathrm{CH}, 3-\mathrm{C}), 128.65$ (CH, 5-C), 131.0 (quat., 4'-C), 138.3 (quat., 2-C), 139.3 (quat., 1'-C), 141.0 (quat., 4-C), 144.3 (quat., 2'-C), 152.2 (quat., 5'-C), 156.6 (quat., 1-C)

\subsubsection{3-(2',4'-Dinitrophenoxy)-2,5-dimethoxybiphenyl 6f}

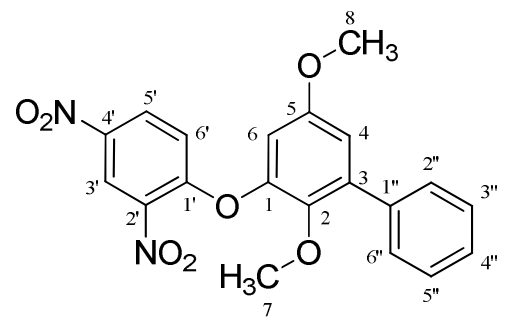

Prepared from 2,5-dimethoxybiphenyl-3-ol 4f (0.98g, 4.25mmol). 3-(2',4'-Dinitrophenoxy)2,5-dimethoxybiphenyl 6f was isolated as a cream solid (1.55g, 3.96mmol, 91.8\%). Recrystallization from $\mathrm{EtOH}$ yielded cream coloured plates; $\mathrm{mp} 146-148^{\circ} \mathrm{C}$; (found: $\mathrm{C}, 60.6$; $\mathrm{H}, 4.1 ; \mathrm{N}, 7.0 \% . \mathrm{C}_{20} \mathrm{H}_{16} \mathrm{~N}_{2} \mathrm{O}_{7}$ requires $\left.\mathrm{C}, 60.6 ; \mathrm{H}, 4.1 ; \mathrm{N}, 7.1 \%\right) ; m / Z 397.1(M H)^{+}, 419.1$ $(M N a)^{+} ; v_{\max } / \mathrm{cm}^{-1} 1610(\mathrm{C}=\mathrm{C}), 1529$ and $1337\left(\mathrm{NO}_{2}\right), 1271$ and $1048(\mathrm{C}-\mathrm{O}) ; \delta_{H}(300 \mathrm{MHz}$, $\left.\mathrm{CDCl}_{3}\right) 3.32\left(3 \mathrm{H}, \mathrm{s}, \mathrm{OCH}_{3}, 7-\mathrm{H}\right), 3.75\left(3 \mathrm{H}, \mathrm{s}, \mathrm{OCH}_{3}, 8-\mathrm{H}\right), 6.68(1 \mathrm{H}, \mathrm{d}, J=3.0 \mathrm{~Hz}, 6-\mathrm{H}), 6.81$ 
$(1 \mathrm{H}, \mathrm{d}, J=3.0 \mathrm{~Hz}, 4-\mathrm{H}), 7.01(1 \mathrm{H}, \mathrm{d}, J=9.3 \mathrm{~Hz}, 6$ '-H), 7.26-7.37 (3H, m, 3"-H, 5"-H and 4"H), $7.42\left(2 \mathrm{H}, \mathrm{m}, 2^{\prime \prime}-\mathrm{H}\right.$ and 6"-H), $8.25\left(1 \mathrm{H}, \mathrm{dd}, J=9.3,2.7 \mathrm{~Hz}, 5^{\prime}-\mathrm{H}\right), 8.76(1 \mathrm{H}, \mathrm{d}, J=2.7 \mathrm{~Hz}$, 3'-H); $\delta_{C}\left(75 \mathrm{MHz}, \mathrm{CDCl}_{3}\right) 55.95\left(\mathrm{OCH}_{3}, 8-\mathrm{C}\right), 61.4\left(\mathrm{OCH}_{3}, 7-\mathrm{C}\right), 107.2(\mathrm{CH}, 6-\mathrm{C}), 114.3$ (CH, 4-C), 117.7 (CH, 6'-C), 121.95 (CH, 3'-C), 128.0 (CH, 4"-C), 128.5 (CH, 3"-C and 5"C), 128.9 (CH, 5'-C), 129.0 (CH, 2"-C and 6"-C), 136.9 (quat., 3-C), 138.1 (quat., 1"-C), 138.6 (quat., 4'-C), 141.4 (quat., 2'-C), 142.7 (quat., 2-C), 146.7 (quat., 1-C), 156.1 (quat., 1'C), 156.4 (quat., 5-C).

\subsection{Preparation of 2,5-dinitro-1-fluorobenzene 13}

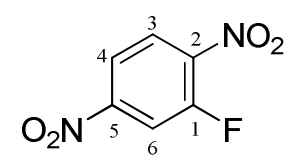

\subsubsection{Nitration of 2-fluoroacetanilide}

A 500mL 3 necked flask equipped with a dropping funnel and a thermometer was charged with 2-fluoroacetanilide $(16.87 \mathrm{~g}, 0.110 \mathrm{~mol})$ and glacial acetic acid $(90 \mathrm{~mL})$. The resulting solution was cooled to $10^{\circ} \mathrm{C}$ ( $\left.\mathrm{T}_{\text {int. }}\right)$. The dropping funnel was charged with a solution of fuming $\mathrm{HNO}_{3}(55 \mathrm{~mL})$ and conc. $\mathrm{H}_{2} \mathrm{SO}_{4}(55 \mathrm{~mL})$, which was added dropwise to the 2fluoroacetanilide solution. The temperature was maintained at $10^{\circ} \mathrm{C}$ during the addition $(1 \mathrm{~h})$. The ice-bath was then removed and the resulting solution was stirred for a further $1 \mathrm{~h} 30 \mathrm{~min}$. The reaction mixture was poured into ice, resulting in the formation of a cream coloured precipitate. The precipitate was recovered by filtration, washed several times with water until the filtrate was of a clear colour, and dried under vacuum. The resulting solid was partially redissolved in $\mathrm{EtOH}(250 \mathrm{~mL})$ and $\mathrm{NaOH}(10 \%, 150 \mathrm{~mL})$ added. The resulting orange solution was stirred for $5 \mathrm{~h}$ or until the acetyl product was fully hydrolysed. The reaction was then quenched with $\mathrm{HCl}(10 \%, 200 \mathrm{~mL})$ resulting in the formation of a yellow precipitate. The precipitate was recovered by filtration, washed several times with water and dried. It was then redissolved into EtOAc, dried $\left(\mathrm{MgSO}_{4}\right)$ and purified by column chromatography, eluting with petroleum ether $\left(60-80^{\circ} \mathrm{C}\right)$ : EtOAc $(70: 30)$. The more mobile band yielded 6-nitro-2fluoroaniline $(2.64 \mathrm{~g}, 16.91 \mathrm{mmol}, 15.3 \%)$ as a yellow solid. Recrystallisation from EtOH produced yellow plates; mp $71.5-72.5^{\circ} \mathrm{C}$ [1it. mp 75.5-76.5 $\left.{ }^{\circ} \mathrm{C}\right]^{7}$; (found: $\mathrm{C}, 46.0 ; \mathrm{H}, 3.2 ; \mathrm{N}$, $17.9 \% . \mathrm{C}_{6} \mathrm{H}_{5} \mathrm{FN}_{2} \mathrm{O}_{2}$ requires $\left.\mathrm{C}, 46.2 ; \mathrm{H}, 3.2 ; \mathrm{N}, 17.9 \%\right) ; v_{\max } / \mathrm{cm}^{-1} 3487$ and $3366\left(\mathrm{NH}_{2}\right), 1583$ $(\mathrm{C}=\mathrm{C}), 1525$ and $1329\left(\mathrm{NO}_{2}\right), 1068(\mathrm{C}-\mathrm{F}) ; \delta_{H}\left(300 \mathrm{MHz}, \mathrm{d}_{6}-\mathrm{DMSO}\right) 6.62(1 \mathrm{H}, \mathrm{td}, J=8.7$, $5.4 \mathrm{~Hz}, 4-\mathrm{H}), 7.22\left(2 \mathrm{H}\right.$, br. s, $\left.\mathrm{NH}_{2}\right), 7.42(1 \mathrm{H}, \mathrm{dd}, J=10.2,7.8 \mathrm{~Hz}, 3-\mathrm{H}), 7.82(1 \mathrm{H}, \mathrm{d}, J=$ 8.7Hz, 5-H); $\delta_{C}\left(75 \mathrm{MHz}, \mathrm{d}_{6}\right.$-DMSO) $113.95(\mathrm{CH}, \mathrm{d}, J=7.9 \mathrm{~Hz}, 4-\mathrm{C}), 120.2(\mathrm{CH}, \mathrm{d}, J=$ 
18.3Hz, 3-C), 121.5 (CH, d, $J=3.3 \mathrm{~Hz}, 5-\mathrm{C}$ ), 132.4 (quat., d, $J=5.1 \mathrm{~Hz}, 6-\mathrm{C}$ ), 136.45 (quat., d, $J=16.6 \mathrm{~Hz}, 1-\mathrm{C}$ ), 152.2 (quat., d, $J=242.7 \mathrm{~Hz}, 2-\mathrm{C}$ ). Further elution gave 4-nitro-2fluoroaniline $(11.61 \mathrm{~g}, 74.37 \mathrm{mmol}, 67.5 \%)$ as a bright yellow crystalline solid. Recrystallization from ethanol gave small yellow needles; mp $130-131^{\circ} \mathrm{C}$ [lit. mp 135$\left.136^{\circ} \mathrm{C}\right]^{5} ; v_{\max } / \mathrm{cm}^{-1} 3493$ and $3395\left(\mathrm{NH}_{2}\right), 1626(\mathrm{C}=\mathrm{C}), 1523$ and $1314\left(\mathrm{NO}_{2}\right), 1079(\mathrm{C}-\mathrm{F}) ; \delta_{H}$ (300 MHz, d 6 -DMSO) 6.77 (2H, br. s, $\left.\mathrm{NH}_{2}\right), 6.85(1 \mathrm{H}, \mathrm{t}, J=8.7 \mathrm{~Hz}, 6-\mathrm{H}), 7.87-7.93(2 \mathrm{H}, \mathrm{m}$, 3-H and 5-H); $\delta_{C}\left(75 \mathrm{MHz}, \mathrm{d}_{6}\right.$-DMSO) $111.8(\mathrm{CH}, \mathrm{d}, J=22.3 \mathrm{~Hz}, 3-\mathrm{C}), 114.3(\mathrm{CH}, \mathrm{d}, J=$ $5.4 \mathrm{~Hz}, 6-\mathrm{C}$ ), 122.7 (CH, d, $J=1.8 \mathrm{~Hz}, 5-\mathrm{C}$ ), 135.3 (quat., $\mathrm{d}, J=7.8 \mathrm{~Hz}, 4-\mathrm{C}$ ), 144.8 (quat., $\mathrm{d}, J$ $=13.1 \mathrm{~Hz}, 1-\mathrm{C}$ ), 148.4 (quat., $\mathrm{d}, J=241.2 \mathrm{~Hz}, 2-\mathrm{C}$ ).

\subsubsection{Oxidation of 2-fluoro-4-nitroaniline}

In a 2L 3 necked flask equipped with a pressure equalizing dropping funnel and a thermometer, urea hydrogen peroxide adduct $(45.93 \mathrm{~g}, 0.488 \mathrm{~mol})$ was suspended in acetonitrile $(400 \mathrm{~mL})$ and the resulting suspension was cooled with an ice and salt slurry to $10^{\circ} \mathrm{C}$ ( $\left.\mathrm{T}_{\text {int. }}\right)$. The pressure equalizing dropping funnel was charged with a solution of trifluoroacetic anhydride $(61.7 \mathrm{~mL}, 0.444 \mathrm{~mol})$ in acetonitrile $(100 \mathrm{~mL})$, which was added dropwise to the urea hydrogen peroxide adduct suspension; the addition rate was adjusted to keep the temperature below $5^{\circ} \mathrm{C}$. The resulting clear solution was stirred at $-5^{\circ} \mathrm{C}$ for $45 \mathrm{~min}$. A solution of 2-fluoro-4-nitroaniline $(9.90 \mathrm{~g}, 63.40 \mathrm{mmol})$ in acetonitrile $(400 \mathrm{~mL})$ was added dropwise via the pressure equalizing dropping funnel to the trifluoroperacetic acid solution. The addition rate was adjusted to keep the temperature close to $-5^{\circ} \mathrm{C}$; the solution colour ranged from emerald green to blue during the addition of 2-fluoro-4-nitroaniline. Upon completion of the addition, the cooling bath was removed and the resulting solution left with stirring at ambient temperature overnight. Most of the acetonitrile was then removed under reduced pressure and the acidic residue was neutralised by addition of $\mathrm{Na}_{2} \mathrm{CO}_{3}(10 \%)$, until no more evolution of $\mathrm{CO}_{2}$ was observed. The resulting mixture was extracted with $\mathrm{Et}_{2} \mathrm{O}(3 \mathrm{x}$ $300 \mathrm{~mL})$ and the combined organic layers washed with $\mathrm{NaHCO}_{3}(5 \%, 2 \times 250 \mathrm{~mL})$, water $(200 \mathrm{~mL})$, brine $(200 \mathrm{~mL})$, and dried $\left(\mathrm{MgSO}_{4}\right)$. The solvent was removed under reduced pressure and the residue recrystallised from petroleum ether $\left(60-80^{\circ} \mathrm{C}\right)$ using decolourising charcoal, yielding 2,5-dinitro-1-fluorobenzene $\mathbf{1 3}$ as large pale yellow plates $(6.97 \mathrm{~g}$, $37.45 \mathrm{mmol}, 59.1 \%$ ); $\mathrm{mp} 72-74^{\circ} \mathrm{C}$ [lit. $\mathrm{mp} 74-75^{\circ} \mathrm{C}$ (from hexane) $]^{8}$; (found: $\mathrm{C}, 38.5 ; \mathrm{H}, 1.6$; $\mathrm{N}, \quad 14.7 \% . \quad \mathrm{C}_{6} \mathrm{H}_{3} \mathrm{FN}_{2} \mathrm{O}_{4}$ requires $\left.\mathrm{C}, \quad 38.7 ; \quad \mathrm{H}, \quad 1.6 ; \quad \mathrm{N}, 15.05 \%\right) ; \quad v_{\max } /$ $\mathrm{cm}^{-1} 3118(\mathrm{C}-\mathrm{H}), 1549$ and $1343\left(\mathrm{NO}_{2}\right) ; \delta_{H}\left(300 \mathrm{MHz}, \mathrm{d}_{6}\right.$-DMSO) 8.26 (1H, ddd, $J=9.0,2.4$, $1.5 \mathrm{~Hz}, 4-\mathrm{H}), 8.42(1 \mathrm{H}, \mathrm{dd}, J=9.0,7.5 \mathrm{~Hz}, 3-\mathrm{H}), 8.53(1 \mathrm{H}, \mathrm{dd}, J=10.5,2.4 \mathrm{~Hz}, 6-\mathrm{H}) ; \delta_{C}(75$ 
MHz, d d $^{-}$DMSO) 115.05 (CH, d, $\left.J=26.0 \mathrm{~Hz}, 6-\mathrm{C}\right), 120.75$ (CH, d, $\left.J=4.45 \mathrm{~Hz}, 4-\mathrm{C}\right), 128.2$ (CH, d, $J=2.5 \mathrm{~Hz}, 3-\mathrm{C}$ ), 141.2 (quat., d, $J=7.9 \mathrm{~Hz}, 5-\mathrm{C}$ ), 151.2 (quat., d, $J=8.5 \mathrm{~Hz}, 2-\mathrm{C}$ ), 154.8 (quat., d, $J=264.9 \mathrm{~Hz}, 1-\mathrm{C}$ ).

\subsection{Synthesis of diphenylethers $14 a-b$}

\subsubsection{General procedure}

The appropriately substituted phenol (1mol equivalent) was dissolved in dry DMF $(3 \mathrm{~mL} / \mathrm{mmol}$ of substituted phenol) in an oven dried two necked flask equipped with reflux condenser and $\mathrm{CaCl}_{2}$ drying tube. $\mathrm{NaH}$ in an oil dispersion $60 \% \mathrm{w} / \mathrm{w}(2.2 \mathrm{~mol}$ equivalent $)$ was then added portion-wise and, once evolution of $\mathrm{H}_{2}$ had stopped, a solution of 2,5dinitrofluorobenzene 13 (1mol equivalent) was added in DMF $(3 \mathrm{~mL} / \mathrm{mmol}$ of $2,5-$ dinitrofluorobenzene). The resulting deep red solution was left with stirring for $1 \mathrm{~h}$ at $\mathrm{rt}$ and then heated at $50^{\circ} \mathrm{C}$ for another $5 \mathrm{~h}$. The reaction was then allowed to cool to $\mathrm{rt}$ and quenched with brine $(2 \mathrm{~mL} / \mathrm{mL}$ of $\mathrm{DMF})$. The resulting mixture was extracted with $\mathrm{DCM}(3 \times$ $0.5 \mathrm{~mL} / \mathrm{mL}$ of suspension), the combined DCM layers washed with water $(0.5 \mathrm{~mL} / \mathrm{mL}$ of DCM extract), brine $(2 \times 0.5 \mathrm{~mL} / \mathrm{mL}$ of DCM extract $)$ and then dried $\left(\mathrm{MgSO}_{4}\right)$. The solvent was removed in vacuo and the residue subjected to column chromatography, eluting with a solvent mixture of petroleum ether $\left(60: 80^{\circ} \mathrm{C}\right)$ : EtOAc. The product was recrystallized from $\mathrm{EtOH}$.

\subsubsection{2,5-Dinitro-4'-'butyl-2',5'-dimethoxydiphenylether 14a}

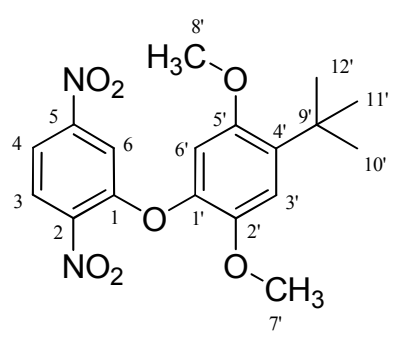

Prepared from 2,5-dimethoxy-4-'butylphenol 4d (1.88g, 8.92mmol). 2,5-Dinitro-4'- ${ }^{t}$ butyl2',5'-dimethoxydiphenylether 14a was isolated as an orange solid $(1.59 \mathrm{~g}, 4.23 \mathrm{mmol}, 47.4 \%)$. Recrystallization from EtOH produced bright orange plates; mp $(\mathrm{EtOH}) 138^{\circ} \mathrm{C}$; (found: $\mathrm{C}$, 57.4; H, 5.35; N, 7.25\%. $\mathrm{C}_{18} \mathrm{H}_{20} \mathrm{~N}_{2} \mathrm{O}_{7}$ requires C, 57.4; H, 5.4; N, 7.4\%); m/z $399.1(M N a)^{+}$; $v_{\max } / \mathrm{cm}^{-1} 1586(\mathrm{C}=\mathrm{C}), 1537$ and $1348\left(\mathrm{NO}_{2}\right), 1206$ and $1038(\mathrm{C}-\mathrm{O}) ; \delta_{H}\left(300 \mathrm{MHz}, \mathrm{CDCl}_{3}\right)$ $1.33\left(9 \mathrm{H}, \mathrm{s}, \mathrm{C}\left(\mathrm{CH}_{3}\right)_{3}\right), 3.64\left(3 \mathrm{H}, \mathrm{s}, \mathrm{OCH}_{3}, 7^{\prime}-\mathrm{H}\right), 3.72\left(3 \mathrm{H}, \mathrm{s}, \mathrm{OCH}_{3}, 8^{\prime}-\mathrm{H}\right), 6.66(1 \mathrm{H}, \mathrm{s}, 6$ '- $-\mathrm{H})$, $6.94(1 \mathrm{H}, \mathrm{s}, 3 \mathrm{\prime}-\mathrm{H}), 7.56(1 \mathrm{H}, \mathrm{d}, J=3.0 \mathrm{~Hz}, 6-\mathrm{H}), 7.82(1 \mathrm{H}, \mathrm{dd}, J=8.7,2.1 \mathrm{~Hz}, 4-\mathrm{H}), 7.92$ $(1 \mathrm{H}, \mathrm{d}, J=8.7 \mathrm{~Hz}, 3-\mathrm{H}) ; \delta_{C}\left(75 \mathrm{MHz}, \mathrm{CDCl}_{3}\right) 29.7\left(\mathrm{CH}_{3}, 10^{\prime} / 11^{\prime} / 12^{\prime}-\mathrm{C}\left(\mathrm{CH}_{3}\right)_{3}\right), 35.2$ (quat., 9'- 
$\left.\mathrm{C}\left(\mathrm{CH}_{3}\right)_{3}\right), 55.8\left(\mathrm{OCH}_{3}, 8^{\prime}-\mathrm{C}\right), 57.0\left(\mathrm{OCH}_{3}, 7^{\prime}-\mathrm{C}\right), 106.5\left(\mathrm{CH}, 6{ }^{\prime}-\mathrm{C}\right), 112.8(\mathrm{CH}, 6-\mathrm{C}), 113.8$ (CH, 3'-C), 116.3 (CH, 4-C), 126.1 (CH, 3-C), 137.9 (quat., 4'-C), 139.7 (quat., 1'-C), 142.8 (quat., 5-C), 143.9 (quat., 2'-C), 150.4 (quat., 2-C), 152.3 (quat., 1-C), 153.4 (quat., 5'-C).

\subsubsection{2,5-Dinitro-2',5'-dimethoxy-4'-pentyldiphenylether 14b}

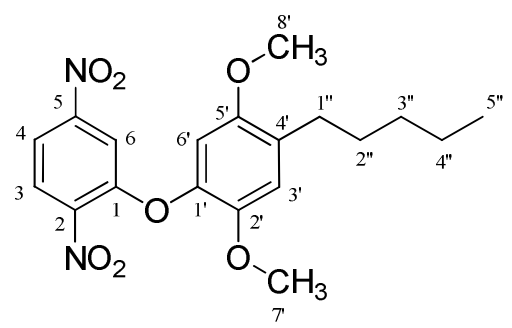

Prepared from 2,5-dimethoxy-4-pentylphenol 4e (2.07g, 9.25mmol). The product was purified by column chromatography, eluting with petroleum ether $\left(60-80^{\circ} \mathrm{C}\right): \mathrm{Et}_{2} \mathrm{O}$ (75:25). 2,5Dinitro-2',5'-dimethoxy-4'-pentyldiphenylether 14b was isolated as a bright orange solid (1.69g, 4.33mmol, 46.8\%). Recrystallization from EtOH produced small yellow needles; mp 77-79 ${ }^{\circ} \mathrm{C}$; (found: $\mathrm{C}, 58.3 ; \mathrm{H}, 5.65 ; \mathrm{N}, 7.2 \% . \mathrm{C}_{19} \mathrm{H}_{22} \mathrm{~N}_{2} \mathrm{O}_{7}$ requires $\mathrm{C}, 58.5 ; \mathrm{H}, 5.7 ; \mathrm{N}, 7.2 \%$ ); $m / Z 391.2(M H)^{+}, 413.2(M N a)^{+} ; v_{\max } / \mathrm{cm}^{-1} 2853-2952(\mathrm{C}-\mathrm{H}), 1629(\mathrm{C}=\mathrm{C}), 1531$ and 1347 $\left(\mathrm{NO}_{2}\right), 1209$ and $1038(\mathrm{C}-\mathrm{O}) ; \delta_{H}\left(300 \mathrm{MHz}, \mathrm{CDCl}_{3}\right) 0.84(3 \mathrm{H}, \mathrm{t}, J=6.9 \mathrm{~Hz}, 5 "-\mathrm{H}), 1.25-1.30$ (4H, m, 3"-H and 4"-H), 1.48-1.55 (2H, m, 2"-H), 2.54 (2H, t, $J=8.1 \mathrm{~Hz}, 1 "-\mathrm{H}), 3.63$ (3H, s, $\left.\mathrm{OCH}_{3}, 7^{\prime}-\mathrm{H}\right), 3.70$ (3H, s, $\left.\mathrm{OCH}_{3}, 8^{\prime}-\mathrm{H}\right), 6.63\left(1 \mathrm{H}, \mathrm{s}, 6^{\prime}-\mathrm{H}\right), 6.785\left(1 \mathrm{H}, \mathrm{s}, 3^{\prime}-\mathrm{H}\right), 7.53(1 \mathrm{H}, \mathrm{d}, J$ $=2.4 \mathrm{~Hz}, 6-\mathrm{H}), 7.83(1 \mathrm{H}, \mathrm{dd}, J=9.0,2.4 \mathrm{~Hz}, 4-\mathrm{H}), 7.93(1 \mathrm{H}, \mathrm{d}, J=8.7 \mathrm{~Hz}, 3-\mathrm{H}) ; \delta_{C}(75 \mathrm{MHz}$, $\left.\mathrm{CDCl}_{3}\right) 14.0\left(\mathrm{CH}_{3}, 5\right.$ "-C), $22.6\left(\mathrm{CH}_{2}, 3\right.$ "-C or 4"-C), $29.6\left(\mathrm{CH}_{2}, 2^{2}-\mathrm{C}\right), 30.1\left(\mathrm{CH}_{2}, 1\right.$ "-C $), 31.8$ $\left(\mathrm{CH}_{2}, 3\right.$ "-C or 4"-C), $56.1\left(\mathrm{OCH}_{3}, 8^{\prime}-\mathrm{C}\right), 56.9\left(\mathrm{OCH}_{3}, 7^{\prime}-\mathrm{C}\right), 105.6(\mathrm{CH}, 6$ '-C), $112.6(\mathrm{CH}, 6-$ C), 115.9 (CH, 3'-C), 116.25 (CH, 4-C), 126.1 (CH, 3-C), 130.8 (quat., 4'-C), 139.5 (quat., 1'C), 142.8 (quat., 5-C), 144.4 (quat., 2'-C), 150.4 (quat., 2-C), 152.2 (quat., 5'-C), 152.4 (quat., $1-\mathrm{C})$.

\subsection{Synthesis of dihydroxydiphenylethers $7 \mathrm{a}-\mathrm{b}$}

\subsubsection{General procedure for the demethylation of 2,4-dinitro-2',5'-dimethoxydiphenyl} ethers 6a-b

In a flame dried 2 necked flask equipped with a thermometer and flushed with $\mathrm{N}_{2}$, the substituted biarylether (1mol equivalent) was dissolved in dry DCM $(10 \mathrm{~mL} / \mathrm{mmol}$ of biarylether). The resulting solution was cooled to $-78^{\circ} \mathrm{C}$ in a dry ice/acetone bath and a $1 \mathrm{M}$ DCM solution of $\mathrm{BBr}_{3}$ (6mol equivalents) was slowly added to the biarylether solution. The addition rate was adjusted in order to keep the temperature below $-55^{\circ} \mathrm{C}$. The resulting 
solution was maintained at $-78^{\circ} \mathrm{C}$ for $40 \mathrm{~min}$, then was allowed to warm up to rt and left with stirring overnight under a nitrogen atmosphere. After 18-20 hours, the solution was cooled again to $-78^{\circ} \mathrm{C}$ and further $1 \mathrm{M} \mathrm{DCM}$ solution of $\mathrm{BBr}_{3}$ (3 mol equivalents) was added. The resulting solution was allowed to warm to rt and stirred for a further 5 hours, then quenched with ice to neutralise the excess $\mathrm{BBr}_{3}$, and the $\mathrm{DCM}$ was removed under reduced pressure. The resulting mixture was extracted with diethyl ether $(3 \times 10 \mathrm{~mL} / \mathrm{mmol}$ of biarylether $)$ and the combined organic extracts were washed once with brine $(0.5 \mathrm{~mL} / \mathrm{mL}$ of diethyl ether extract) and dried $\left(\mathrm{MgSO}_{4}\right)$. The solvent was removed in vacuo and the residue subjected to column chromatography.

\subsubsection{2,4-Dinitro-2',5'-dihydroxydiphenylether 7a}

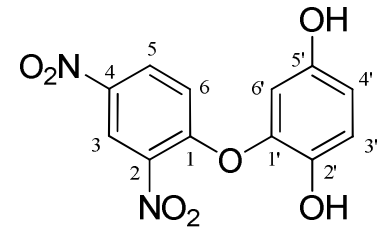

The title compound was prepared as described from 2,4-dinitro-2',5'-dimethoxydiphenylether 6a $(4.01 \mathrm{~g}, 12.50 \mathrm{mmol})$. The product was purified by column chromatography, eluting with petroleum ether $\left(60-80^{\circ} \mathrm{C}\right)$ : EtOAc (30:70). 2,4-Dinitro-2',5'-dihydroxydiphenylether 7a was isolated as an amorphous hygroscopic yellow solid (3.53g, 12.10mmol, 96.7\%) and further purified by hot trituration with DCM; mp $113-115^{\circ} \mathrm{C}$; (found: $\mathrm{C}, 49.0 ; \mathrm{H}, 2.8 ; \mathrm{N}, 9.55 \%$. $\mathrm{C}_{12} \mathrm{H}_{8} \mathrm{~N}_{2} \mathrm{O}_{6}$ requires $\left.\mathrm{C}, 49.3 ; \mathrm{H}, 2.8 ; \mathrm{N}, 9.6 \%\right) ; \mathrm{m} / \mathrm{Z} 243.9\left(M-H_{2} \mathrm{NO}_{2}\right)^{-}, 290.9(M-H)^{-} ; \mathrm{v}_{\max } / \mathrm{cm}^{-}$

${ }^{1} 3401$ and $3347(\mathrm{OH}), 1600(\mathrm{C}=\mathrm{C}), 1506$ and $1340\left(\mathrm{NO}_{2}\right) ; \delta_{H}\left(300 \mathrm{MHz}, \mathrm{d}_{6}-\mathrm{DMSO}\right) 6.29$ $\left(1 \mathrm{H}, \mathrm{dd}, J=8.7,2.7 \mathrm{~Hz}, 4^{\prime}-\mathrm{H}\right), 6.49\left(1 \mathrm{H}, \mathrm{d}, J=2.7 \mathrm{~Hz}, 6^{\prime}-\mathrm{H}\right), 6.97(1 \mathrm{H}, \mathrm{d}, J=3.3 \mathrm{~Hz}, 6-\mathrm{H})$, $7.00\left(1 \mathrm{H}, \mathrm{d}, J=2.7 \mathrm{~Hz}, 3^{\prime}-\mathrm{H}\right), 8.39(1 \mathrm{H}, \mathrm{dd}, J=9.3,3.0 \mathrm{~Hz}, 5-\mathrm{H}), 8.82(1 \mathrm{H}, \mathrm{d}, J=3.0 \mathrm{~Hz}, 3-\mathrm{H})$, $9.49\left(1 \mathrm{H}, \mathrm{s}, 5\right.$ '-OH), $9.84\left(1 \mathrm{H}, \mathrm{s}, 2^{\prime}-\mathrm{OH}\right) ; \delta_{C}\left(75 \mathrm{MHz}, \mathrm{d}_{6}-\mathrm{DMSO}\right) 104.8$ (CH, 6'-C ), 107.2 (CH, 4'-C), 117.6 (CH, 6-C), 122.1 (CH, 3-C), 123.2 (CH, 3'-C ), 129.8 (CH, 5-C ), 133.0 (quat., 2'-C ), 138.5 (quat., 2-C ), 140.85 (quat., 4-C ), 149.8 (quat., 1'-C ), 156.7 (quat., 5'-C), 157.1 (quat., 1-C). 


\subsubsection{2,4-Dinitro-2',5'-dihydroxy-3',4',6'-trimethyldiphenylether 7b and 2,4- dinitrophenoxy-3',4',6'-trimethyl-2',5'-benzoquinone 8a}
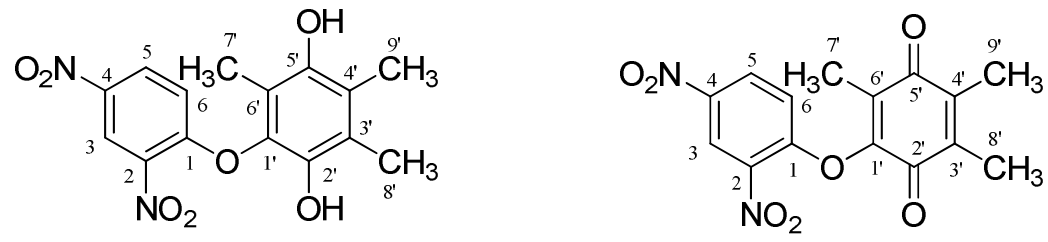

The title compounds were prepared as described from 2,4-dinitro-2',5'-dimethoxy-3',4',6'trimethyldiphenylether $\mathbf{6 b}(3.38 \mathrm{~g}, 9.32 \mathrm{mmol})$; elution used petroleum ether $\left(60-80^{\circ} \mathrm{C}\right): \mathrm{Et}_{2} \mathrm{O}$ (70:30). Ambient evaporation of the solvent until crystals of 2,4-dinitro-2',5'-dihydroxy3',4',6'-trimethyldiphenylether $\mathbf{7 b}$ formed, and collection by filtration, yielded 2,4-dinitro2',5'-dihydroxy-3',4',6'-trimethyldiphenylether $7 \mathbf{b} \quad(1.67 \mathrm{~g}, 4.99 \mathrm{mmol}, 53.5 \%)$ as cream coloured prisms; mp $131-139^{\circ} \mathrm{C}$; (found: $\mathrm{C}, 53.8 ; \mathrm{H}, 4.2 ; \mathrm{N}, 8.3 \% . \mathrm{C}_{15} \mathrm{H}_{14} \mathrm{~N}_{2} \mathrm{O}_{7}$ requires $\mathrm{C}$, 53.9; H, 4.2; N, 8.4\%); m/Z $285.9\left(M-H_{2} N O_{2}\right)^{-}, 332.9(M-H)^{-} ; v_{\max } / \mathrm{cm}^{-1} 3431(\mathrm{O}-\mathrm{H}), 1602$ and $1517(\mathrm{C}=\mathrm{C}), 1537$ and $1348\left(\mathrm{NO}_{2}\right), 1244(\mathrm{C}-\mathrm{O}) ; \delta_{H}\left(300 \mathrm{MHz}, \mathrm{d}_{6}-\mathrm{DMSO}\right) 1.99\left(3 \mathrm{H}, \mathrm{s}, \mathrm{CH}_{3}\right.$, 7'-H), 2.09 (6H, s, $2 \times \mathrm{CH}_{3}, 8^{\prime}-\mathrm{H}$ and 9'-H), $6.83(1 \mathrm{H}, \mathrm{d}, J=9.6 \mathrm{~Hz}, 6-\mathrm{H}), 7.965\left(1 \mathrm{H}, \mathrm{s}, 5^{\prime}-\right.$ $\mathrm{OH}), 8.40(1 \mathrm{H}, \mathrm{dd}, J=9.6,3.0 \mathrm{~Hz}, 5-\mathrm{H}), 8.79\left(1 \mathrm{H}, \mathrm{s}, 2^{\prime}-\mathrm{OH}\right), 8.84(1 \mathrm{H}, \mathrm{d}, J=3.0 \mathrm{~Hz}, 3-\mathrm{H}) ; \delta_{C}$

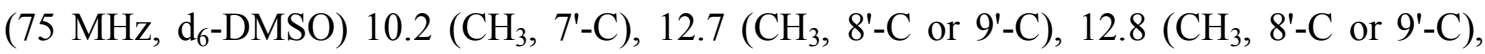
111.9 (quat., 3'-C), 115.8 (quat., 4'-C), 116.85 (CH, 6-C), 122.2 (CH, 3-C), 126.1 (quat., 6'C), 130.0 (CH, 5-C), 132.7 (quat., 1'-C), 138.6 (quat., 4-C), 140.8 (quat., 2-C), 145.1 (quat., 2'-C), 152.0 (quat., 5'-C), 156.6 (quat., 1-C).

Evaporation of the filtrate and recrystallization of the residue from $\mathrm{MeOH}$ provided 2,4dinitrophenoxy-3',4',6'-trimethyl-2',5'-benzoquinone 8a as fluffy orange crystals $(0.40 \mathrm{~g}, 1.22$ mmol, 13.0\%); mp 142-145 ${ }^{\circ}$; (found: $\mathrm{C}, 54.2 ; \mathrm{H}, 3.6 ; \mathrm{N}, 8.4 \% . \mathrm{C}_{12} \mathrm{H}_{8} \mathrm{~N}_{2} \mathrm{O}_{6}$ requires $\mathrm{C}$, 54.2; $\mathrm{H}, 3.6 ; \mathrm{N}, 8.4 \%) ; m / z 355.1(M N a)^{+} ; v_{\max } / \mathrm{cm}^{-1} 1666$ and $1648(\mathrm{C}=\mathrm{O}), 1602(\mathrm{C}=\mathrm{C}), 1526$ and $1343\left(\mathrm{NO}_{2}\right), 1249$ (C-O); $\delta_{H}\left(300 \mathrm{MHz}, \mathrm{d}_{6}\right.$-DMSO) $1.95\left(3 \mathrm{H}, \mathrm{d}, J=1.2 \mathrm{~Hz}, \mathrm{CH}_{3}, 7^{\prime}-\mathrm{H}\right), 1.97$ $\left(3 \mathrm{H}, \mathrm{s}, \mathrm{CH}_{3}, 9 '-\mathrm{H}\right), 2.04\left(3 \mathrm{H}, \mathrm{d}, J=0.9 \mathrm{~Hz}, \mathrm{CH}_{3}, 8^{\prime}-\mathrm{H}\right), 7.54(1 \mathrm{H}, \mathrm{d}, J=9.3 \mathrm{~Hz}, 6-\mathrm{H}), 8.38(1 \mathrm{H}$, dd, $J=9.3,2.7 \mathrm{~Hz}, 5-\mathrm{H}), 8.89(1 \mathrm{H}, \mathrm{d}, J=2.7 \mathrm{~Hz}, 3-\mathrm{H}) ; \delta_{C}\left(75 \mathrm{MHz}, \mathrm{d}_{6}-\mathrm{DMSO}\right) 9.85\left(\mathrm{CH}_{3}, 9^{\prime}-\right.$ C), $12.2\left(\mathrm{CH}_{3}, 7^{\prime}-\mathrm{C}\right), 12.9\left(\mathrm{CH}_{3}, 8^{\prime}-\mathrm{C}\right), 119.2(\mathrm{CH}, 6-\mathrm{C}), 122.3(\mathrm{CH}, 5-\mathrm{C}), 129.7$ (CH, 3-C), 134.3 (quat., 6'-C), 138.6 (quat., 2-C), 139.1 (quat., 4'-C), 141.7 (quat., 3'-C), 142.3 (quat., 4 C), 149.0 (quat., 1'-C), 154.1 (quat., 1-C), 180.5 (quat., 2'-C), 186.9 (quat., 5'-C). 


\subsection{Synthesis of dinitrobenzophenones 8b-8e and 15a-b}

\subsubsection{General procedure for the oxidative-demethylation of 2,4-dinitro-2',5'- dimethoxydiphenylethers 6b, d-f and 2,5-dinitro-2',5'-dimethoxydiphenylethers 14a-b}

The correctly substituted dinitro-2',5'-dimethoxydiphenylether (1mol equivalent) was dissolved in $\mathrm{CH}_{3} \mathrm{CN}(20 \mathrm{~mL} / \mathrm{mmol}$ of diphenylether) in a 2 necked flask equipped with a thermometer and a dropping funnel. Cerium (IV) ammonium nitrate (3mol equivalent) was dissolved in water $(3 \mathrm{~mL} / \mathrm{mmol}$ of cerium (IV) ammonium nitrate) and the resulting solution added slowly to the biaryl ether solution via the dropping funnel. The yellow solution usually darkened upon addition of the cerium (IV) ammonium nitrate solution, followed by the formation of a yellow precipitate soon after the addition was finished. The resulting mixture was stirred for a further $1 \mathrm{~h}$ to ensure completion of the reaction. $\mathrm{CH}_{3} \mathrm{CN}$ was removed under reduced pressure and, whenever possible, the precipitate formed was collected by filtration and washed with water. It was then redissolved in DCM, the resulting solution dried $\left(\mathrm{MgSO}_{4}\right)$ and the solvent was removed in vacuo. The residue was recrystallized from ethanol. When no precipitate, but a yellow oil/syrup, was observed, the reaction mixture was extracted twice with DCM and the combined organic layers washed once with brine and dried $\left(\mathrm{MgSO}_{4}\right)$. The solvent was then removed under reduced pressure and the residue either subjected to column chromatography or recrystallized from EtOH.

\subsubsection{2,4-Dinitrophenoxy-3',4'-dimethyl-2',5'-benzoquinone $8 \mathrm{~b}$}

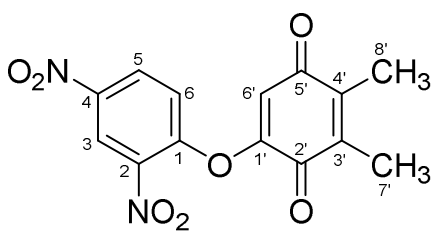

Prepared from 2,4-dinitro-2',5'-dimethoxy-3',4'-dimethyldiphenylether $6 \mathbf{b}(0.57 \mathrm{~g}, 1.63 \mathrm{mmol})$. Elution with petroleum ether $\left(60: 80^{\circ} \mathrm{C}\right)$ : EtOAc (30:70) yielded 2,4-dinitrophenoxy-3',4'dimethyl-2',5'-benzoquinone $\mathbf{8 b}$ as a yellow solid $(0.47 \mathrm{~g}, 1.48 \mathrm{mmol}, 90.9 \%)$. Recrystallization from EtOH produced yellow prisms; mp $110-111^{\circ} \mathrm{C}$; (found: $\mathrm{C}, 52.8 ; \mathrm{H}, 3.2 ; \mathrm{N}, 8.7 \%$. $\mathrm{C}_{14} \mathrm{H}_{10} \mathrm{~N}_{2} \mathrm{O}_{7}$ requires $\left.\mathrm{C}, 52.8 ; \mathrm{H}, 3.2 ; \mathrm{N}, 8.8 \%\right) ; \mathrm{m} / \mathrm{Z} 341.1(M N a)^{+} ; v_{\max } / \mathrm{cm}^{-1} 1666$ and 1648 $\left(\mathrm{C}=\mathrm{O}\right.$, quinone), $1600(\mathrm{C}=\mathrm{C}), 1537$ and $1347\left(\mathrm{NO}_{2}\right), 1190(\mathrm{C}-\mathrm{O}) ; \delta_{H}\left(300 \mathrm{MHz}, \mathrm{d}_{6}-\mathrm{DMSO}\right)$ $2.01\left(6 \mathrm{H}, \mathrm{s}, 2 \times \mathrm{CH}_{3}, 7^{\prime}-\mathrm{H}\right.$ and $\left.8^{\prime}-\mathrm{H}\right), 6.46\left(1 \mathrm{H}, \mathrm{s}, 6{ }^{\prime}-\mathrm{H}\right), 7.77(1 \mathrm{H}, \mathrm{d}, J=9.0 \mathrm{~Hz}, 6-\mathrm{H}), 8.55$ $(1 \mathrm{H}, \mathrm{dd}, J=9.0,2.7 \mathrm{~Hz}, 5-\mathrm{H}), 8.91(1 \mathrm{H}, \mathrm{d}, J=2.7 \mathrm{~Hz}, 3-\mathrm{H}) ; \delta_{C}\left(75 \mathrm{MHz}, \mathrm{d}_{6}-\mathrm{DMSO}\right) 12.3$ $\left(\mathrm{CH}_{3}, 7^{\prime}-\mathrm{C}\right.$ or 8'-C), $12.6\left(\mathrm{CH}_{3}, 7^{\prime}-\mathrm{C}\right.$ or 8'-C), $117.9\left(\mathrm{CH}, 66^{\prime}-\mathrm{C}\right), 122.5(\mathrm{CH}, 3-\mathrm{C}), 123.5(\mathrm{CH}$, 
6-C), 130.3 (CH, 5-C), 139.5 (quat., 3'-C), 140.5 (quat., 2-C), 141.4 (quat., 4'-C), 144.1 (quat., 4-C), 152.1 (quat., 1-C), 155.2 (quat., 1'-C), 180.8 (quat., 2'-C), 187.1 (quat., 5'-C).

\subsubsection{2,4-Dinitrophenoxy-4'-'butyl-2',5'-benzoquinone 8c}

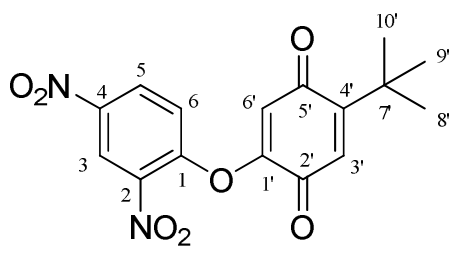

Prepared from 2,4-dinitro-4'-'butyl-2',5'-dimethoxydiphenylether 6d (2.87g, 7.69mmol). The crude product was recrystallized from $\mathrm{MeOH}$ yielding 2,4-dinitrophenoxy-4'-'butyl-2',5'benzoquinone $8 \mathrm{c}$ as a fluffy yellow solid $(1.68 \mathrm{~g}, 4.85 \mathrm{mmol}, 63.0 \%)$; mp $179.5-182.5^{\circ} \mathrm{C}$; (found: $\mathrm{C}, 55.5 ; \mathrm{H}, 4.1 ; \mathrm{N}, 8.1 \% . \mathrm{C}_{16} \mathrm{H}_{14} \mathrm{~N}_{2} \mathrm{O}_{7}$ requires $\mathrm{C}, 55.5 ; \mathrm{H}, 4.1 ; \mathrm{N}, 8.1 \%$ ); $\mathrm{m} / \mathrm{Z} 369.1$ $(M N a)^{+} ; v_{\max } / \mathrm{cm}^{-1} 2870-2957(\mathrm{C}-\mathrm{H}), 1665$ and $1647(\mathrm{C}=\mathrm{O}$, quinone), 1611 and $1591(\mathrm{C}=\mathrm{C})$, 1533 and $1341\left(\mathrm{NO}_{2}\right), 1145(\mathrm{C}-\mathrm{O}) ; \delta_{H}\left(300 \mathrm{MHz}, \mathrm{d}_{6}-\mathrm{DMSO}\right) 1.27\left(9 \mathrm{H}, \mathrm{s}, \mathrm{C}\left(\mathrm{CH}_{3}\right)_{3}\right), 6.40(1 \mathrm{H}$, s, 6'-H), $6.67\left(1 \mathrm{H}, \mathrm{s}, 3^{\prime}-\mathrm{H}\right), 7.82(1 \mathrm{H}, \mathrm{d}, J=9.3 \mathrm{~Hz}, 6-\mathrm{H}), 8.56(1 \mathrm{H}, \mathrm{dd}, J=9.0,2.7 \mathrm{~Hz}, 5-\mathrm{H})$, $8.92(1 \mathrm{H}, \mathrm{d}, J=2.7 \mathrm{~Hz}, 3-\mathrm{H}) ; \delta_{C}\left(75 \mathrm{MHz}, \mathrm{d}_{6}\right.$-DMSO) $29.5\left(\mathrm{CH}_{3}, 8^{\prime} / 9^{\prime} / 10^{\prime}-\mathrm{C}\left(\mathrm{CH}_{3}\right)\right), 35.5$ (quat., 7'- $\left.\mathrm{C}\left(\mathrm{CH}_{3}\right)_{3}\right), 119.6(\mathrm{CH}, 6$ '-C), $122.5(\mathrm{CH}, 3-\mathrm{C}), 123.9(\mathrm{CH}, 6-\mathrm{C}), 130.3(\mathrm{CH}, 3$ '-C or 5-C), 130.5 (CH, 3'-C or 5-C), 140.6 (quat., 2-C), 144.3 (quat., 4-C), 151.8 (quat., 1-C), 154.5 (quat., 1'-C), 156.3 (quat., 4'-C), 181.4 (quat., 2'-C), 187.6 (quat., 5'-C).

\subsubsection{2,4-Dinitrophenoxy-4'-pentyl-2',5'-benzoquinone 8d}

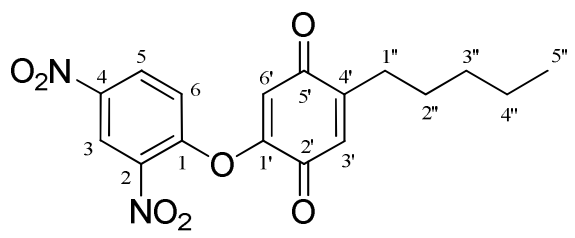

Prepared from 2,4-dinitro-2',5'-dimethoxy-4'-pentyldiphenylether 6e (2.65g, 6.79mmol). Elution with petroleum ether $\left(60-80^{\circ} \mathrm{C}\right)$ : EtOAc (70:30) yielded 2,4-dinitrophenoxy-2',5'dimethoxy-4'-pentylbenzoquinone $\mathbf{8 d}$ as a yellow solid (2.36g, 6.56mmol, 96.7\%). Recrystallization from $\mathrm{EtOH}$ produced shiny yellow plates; mp $110-114^{\circ} \mathrm{C}$; (found: $\mathrm{C}, 56.5$; $\mathrm{H}, 4.4 ; \mathrm{N}, 7.7 \% . \mathrm{C}_{17} \mathrm{H}_{16} \mathrm{~N}_{2} \mathrm{O}_{7}$ requires $\left.\mathrm{C}, 56.7 ; \mathrm{H}, 4.5 ; \mathrm{N}, 7.8 \%\right) ; \mathrm{m} / \mathrm{Z} 383.1(M N a)^{+} ; v_{\max } / \mathrm{cm}^{-1}$ $2927(\mathrm{C}-\mathrm{H}), 1673$ and $1649(\mathrm{C}=\mathrm{O}$, quinone $), 1610(\mathrm{C}=\mathrm{C}), 1530$ and $1342\left(\mathrm{NO}_{2}\right), 1224(\mathrm{C}-\mathrm{O})$; $\delta_{H}\left(300 \mathrm{MHz}, \mathrm{CDCl}_{3}\right) 0.83$ (3H, t, $\left.J=6.9 \mathrm{~Hz}, 5 "-\mathrm{H}\right), 1.26-1.29$ (4H, m, 3"-H and 4"-H), $1.43-$ $1.48(2 \mathrm{H}, \mathrm{m}, 2 "-\mathrm{H}), 2.38(2 \mathrm{H}, \mathrm{td}, J=7.8,1.2 \mathrm{~Hz}, 1 "-\mathrm{H}), 6.03(1 \mathrm{H}, \mathrm{s}, 6 '-\mathrm{H}), 6.53(1 \mathrm{H}, \mathrm{t}, J=$ 
$1.5 \mathrm{~Hz}, 3 \mathrm{\prime}-\mathrm{H}), 7.31(1 \mathrm{H}, \mathrm{d}, J=9.0 \mathrm{~Hz}, 6-\mathrm{H}), 8.43(1 \mathrm{H}, \mathrm{dd}, J=9.0,2.7 \mathrm{~Hz}, 5-\mathrm{H}), 8.88(1 \mathrm{H}, \mathrm{d}, J=$ 2.7Hz, 3-H); $\delta_{C}\left(75 \mathrm{MHz}, \mathrm{CDCl}_{3}\right) 13.9\left(\mathrm{CH}_{3}, 5 "-\mathrm{C}\right), 22.4\left(\mathrm{CH}_{2}, 3\right.$ "-C or 4"-C), $27.6\left(\mathrm{CH}_{2}, 1\right.$ "C), $28.9\left(\mathrm{CH}_{2}, 2\right.$ "-C), $31.45\left(\mathrm{CH}_{2}, 3\right.$ "-C or 4"-C), $116.3(\mathrm{CH}, 6$ '-C), $122.5(\mathrm{CH}, 3-\mathrm{C}), 123.6$ (CH, 6-C), 129.4 (CH, 5-C), 130.6 (CH, 3'-C), 140.7 (quat., 2-C), 144.5 (quat., 4-C), 151.2 (quat., 4'-C), 151.8 (quat., 1-C), 155.3 (quat., 1'-C), 180.3 (quat., 2'-C), 186.6 (quat., 5'-C).

\subsubsection{2,4-Dinitrophenoxy-3'-phenyl-2',5'-benzoquinone $8 \mathrm{e}$}

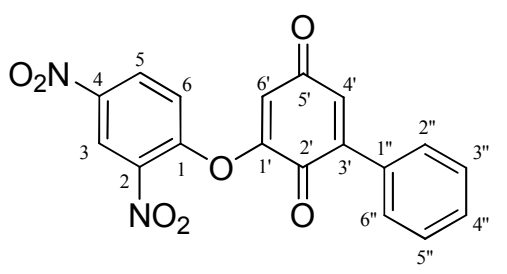

Prepared from 3-(2',4'-dinitrophenoxy)-2,5-dimethoxybiphenyl 6f (1.48g, 3.97mmol). Elution with petroleum ether $\left(60-80^{\circ} \mathrm{C}\right)$ : EtOAc $(20: 80)$ yielded 2,4-dinitrophenoxy-3'-phenyl-2',5'benzoquinone 8e as a yellow crystalline solid (1.23g, 3.36mmol, 84.6\%). Recrystallization from $\mathrm{EtOH} / \mathrm{EtOAc}$ produced golden orange plates; mp $158.5-160^{\circ} \mathrm{C}$; (found: $\mathrm{C}, 58.8 ; \mathrm{H}, 2.8$; $\mathrm{N}, 7.6 \% . \mathrm{C}_{18} \mathrm{H}_{10} \mathrm{~N}_{2} \mathrm{O}_{7}$ requires C, 59.0; H, 2.75; N, 7.65\%); m/Z $389.1(M N a)^{+} ; v_{\max } / \mathrm{cm}^{-1} 1676$ and $1649\left(\mathrm{C}=\mathrm{O}\right.$, quinone), 1590 and $1523(\mathrm{C}=\mathrm{C}), 1543$ and $1347\left(\mathrm{NO}_{2}\right), 1226$ and $1082(\mathrm{C}-$ O); $\delta_{\mathrm{H}}\left(300 \mathrm{MHz}, \mathrm{d}_{6}-\mathrm{DMSO}\right) 6.59\left(1 \mathrm{H}, \mathrm{d}, J=2.4 \mathrm{~Hz}, 6{ }^{\prime}-\mathrm{H}\right), 7.00\left(1 \mathrm{H}, \mathrm{d}, J=2.4 \mathrm{~Hz}, 4^{\prime}-\mathrm{H}\right)$, 7.48-7.51 (3H, m, 3"-H, 5"-H and 4"-H), 7.54-7.58 (2H, m, 2"-H and 6"-H), $7.90(1 \mathrm{H}, \mathrm{d}, J=$ $9.3 \mathrm{~Hz}, 6-\mathrm{H}), 8.60(1 \mathrm{H}, \mathrm{dd}, J=9.3,2.7 \mathrm{~Hz}, 5-\mathrm{H}), 8.94(1 \mathrm{H}, \mathrm{d}, J=2.7 \mathrm{~Hz}, 3-\mathrm{H}) ; \delta_{\mathrm{C}}\left(75 \mathrm{MHz}, \mathrm{d}_{6^{-}}\right.$ DMSO) 117.95 (CH, 6'-C), 122.5 (CH, 3-C), 123.6 (CH, 6-C), 128.8 (CH, 3"-C and 5"-C), 129.8 (CH, 2"-C and 6"-C), 130.4 (CH, 5-C and 4"-C), 132.7 (quat., 1"-C), 133.15 (CH, 4'-C), 140.6 (quat., 2-C), 144.1 (quat., 4-C), 144.7 (quat., 3'-C), 152.2 (quat., 1-C), 155.9 (quat., 1'C), 179.9 (quat., 2'-C), 187.3 (quat., 5'-C).

\subsubsection{2,5-Dinitrophenoxy-4'- ${ }^{t}$ butyl-2',5'-benzoquinone $15 a$}

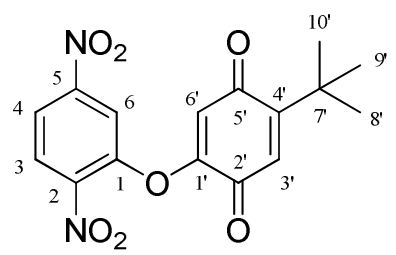

Prepared from 2,5-dinitro-4'-'butyl-2',5'-dimethoxydiphenylether 14a (1.57g, 4.18mmol). 2,5Dinitrophenoxy-4'-'butyl-2',5'-benzoquinone 15a was isolated as a pale yellow solid $(1.35 \mathrm{~g}$, 
$3.89 \mathrm{mmol}, 93.2 \%)$. Recrystallization from EtOH produced a pale yellow microcrystalline solid; mp 165-168 ${ }^{\circ} \mathrm{C}$; (found: $\mathrm{C}, 55.1 ; \mathrm{H}, 4.0 ; \mathrm{N}, 8.0 \% . \mathrm{C}_{16} \mathrm{H}_{14} \mathrm{~N}_{2} \mathrm{O}_{7}$ requires $\mathrm{C}, 55.5 ; \mathrm{H}, 4.1$; $\mathrm{N}, 8.1 \%) ; \mathrm{m} / \mathrm{z} 369.1(\mathrm{MNa})^{+} ; v_{\max } / \mathrm{cm}^{-1} 2952(\mathrm{C}-\mathrm{H}), 1672$ and $1650(\mathrm{C}=\mathrm{O}$, quinone), 1594 $(\mathrm{C}=\mathrm{C}), 1540$ and $1347\left(\mathrm{NO}_{2}\right), 1237(\mathrm{C}-\mathrm{O})$; $\delta_{\mathrm{H}}\left(300 \mathrm{MHz}, \mathrm{d}_{6}-\mathrm{DMSO}\right) 1.26\left(9 \mathrm{H}, \mathrm{s}, \mathrm{C}\left(\mathrm{CH}_{3}\right)_{3}\right)$, $6.19\left(1 \mathrm{H}, \mathrm{s}, 6^{\prime}-\mathrm{H}\right), 6.66\left(1 \mathrm{H}, \mathrm{s}, 3^{\prime}-\mathrm{H}\right), 8.33(1 \mathrm{H}, \mathrm{dd}, J=9.0,2.4 \mathrm{~Hz}, 4-\mathrm{H}), 8.41(1 \mathrm{H}, \mathrm{d}, J=$ $9.0 \mathrm{~Hz}, 3-\mathrm{H}), 8.51(1 \mathrm{H}, \mathrm{d}, J=2.4 \mathrm{~Hz}, 6-\mathrm{H}) ; \delta_{\mathrm{C}}\left(75 \mathrm{MHz}, \mathrm{d}_{6}-\mathrm{DMSO}\right) 29.5\left(3 \times \mathrm{CH}_{3}, 8^{\prime} / 9^{\prime} / 10^{\prime}-\right.$ $\left.\mathrm{C}\left(\mathrm{CH}_{3}\right)_{3}\right), 35.4$ (quat., 7'-C( $\left.\left.\mathrm{CH}_{3}\right)_{3}\right), 116.5\left(\mathrm{CH}, 6{ }^{\prime}-\mathrm{C}\right), 119.9(\mathrm{CH}, 6-\mathrm{C}), 122.3(\mathrm{CH}, 4-\mathrm{C})$, 127.9 (CH, 3-C), 130.4 (CH, 3'-C), 145.3 (quat., 2-C), 146.65 (quat., 5-C), 151.1 (quat., 1-C), 155.8 (quat., 1'-C), 156.1 (quat., 4'-C), 181.4 (quat., 2'-C), 187.8 (quat., 5'-C).

\subsubsection{2,5-Dinitrophenoxy-4'-pentyl-2',5'-benzoquinone $15 \mathrm{~b}$}

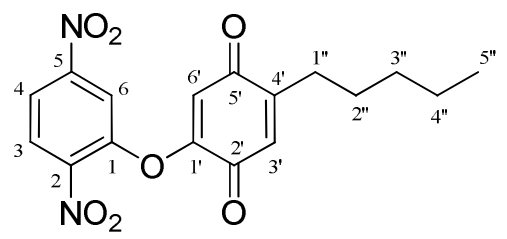

Prepared from 2,4-dinitro-2',5'-dimethoxy-4'-pentyldiphenylether 14b (1.41g, 3.62mmol). Elution with petroleum ether $\left(60-80^{\circ} \mathrm{C}\right)$ : EtOAc (75:25) yielded 2,5-dinitrophenoxy-4'pentyl-2',5'-benzoquinone $\mathbf{1 5 b}$ as a yellow solid $(1.19 \mathrm{~g}, 3.31 \mathrm{mmol}, 91.4 \%)$. Recrystallization from $\mathrm{EtOH}$ produced golden prisms; $\mathrm{mp} 91-94^{\circ} \mathrm{C}$; (found: $\mathrm{C}, 56.4 ; \mathrm{H}, 4.5 ; \mathrm{N}, 7.8 \%$. $\mathrm{C}_{17} \mathrm{H}_{16} \mathrm{~N}_{2} \mathrm{O}_{7}$ requires C, 56.7; H, 4.5; N, 7.8\%); $/ \mathrm{Z}_{\mathrm{Z}} 383.2(M N a)^{+} ; v_{\max } / \mathrm{cm}^{-1} 2870-2957(\mathrm{C}-$ $\mathrm{H}), 1676$ and $1651(\mathrm{C}=\mathrm{O}$, quinone $), 1603(\mathrm{C}=\mathrm{C}), 1544$ and $1343\left(\mathrm{NO}_{2}\right), 1230(\mathrm{C}-\mathrm{O}) ; \delta_{H}(300$ MHz, d 6 -DMSO) 0.79 (3H, t, $J=6.9 \mathrm{~Hz}, 5 "-\mathrm{H}), 1.24-1.19$ (4H, m, 3"-H and 4"-H), 1.42-1.35 (2H, m, 2"-H), 2.29 (2H, t, J=6.9Hz, 1"-H), $6.18(1 \mathrm{H}, \mathrm{s}, 6$ '-H), $6.675(1 \mathrm{H}, \mathrm{s}, 3$ '-H), $8.23(1 \mathrm{H}$, dd, $J=9.0,2.4 \mathrm{~Hz}, 4-\mathrm{H}), 8.32(1 \mathrm{H}, \mathrm{d}, J=9.0 \mathrm{~Hz}, 3-\mathrm{H}), 8.41(1 \mathrm{H}, \mathrm{d}, J=2.4 \mathrm{~Hz}, 6-\mathrm{H}) ; \delta_{C}(75$ MHz, d 6 -DMSO) $14.3\left(\mathrm{CH}_{3}, 5 "-\mathrm{H}\right), 22.3\left(\mathrm{CH}_{2}, 3\right.$ "-C or 4"-C), $27.75\left(\mathrm{CH}_{2}, 2\right.$ "-C), $28.6\left(\mathrm{CH}_{2}\right.$, 1"-C), $31.4\left(\mathrm{CH}_{2}, 3\right.$ "-C or 4"-C), $115.1(\mathrm{CH}, 6$ '-C), 119.8 (CH, 6-C), $122.2(\mathrm{CH}, 4-\mathrm{C}), 127.9$ (CH, 3-C), 131.2 (CH, 3'-C), 145.25 (quat., 2-C), 146.7 (quat., 5-C), 150.2 (quat., 4'-C), 151.1 (quat., 1-C), 156.6 (quat., 1'-C), 181.0 (quat., 2'-C), 187.65 (quat., 5'-C).

\subsection{Trapping of diamino-dihydroxydiphenyl ether intermediates 9a, 11b-d and 16a}

\subsubsection{General procedure}

Either 2,4-dinitro-2',5'-dihydroxydiphenylether 9a (1mol equivalent) or the correctly substituted dinitrophenoxy-2',5'-benzoquinone 11b-d and 16a (1mol equivalent) was dissolved in $\mathrm{MeOH}(10 \mathrm{~mL} / \mathrm{mmol}$ of 2,4-dinitro-2',5'-dihydroxydiphenylether) or an 
EtOAc:MeOH (1:1) solvent mixture $(20 \mathrm{~mL} / \mathrm{mmol}$ of dinitrophenoxy-2',5'-benzoquinone) and $\mathrm{Pd} / \mathrm{C} 5 \%(10 \% \mathrm{w} / \mathrm{w}$ of starting material $)$ was added to the resulting solution. The resulting mixture was hydrogenated in a Berghof apparatus for $4 \mathrm{~h}$ at a $\mathrm{H}_{2}$ pressure of $2.4 \mathrm{bar}$. Acetic anhydride (10mol equivalent) was added as soon as the hydrogenation was stopped and the resulting mixture was stirred at ambient atmosphere overnight. The reaction mixture was filtered through a pad of Celite, which was washed several times with $\mathrm{MeOH}$ and the filtrate evaporated in vacuo. $\mathrm{NaHCO}_{3}(5 \%)$ was added to the residue and the resulting mixture extracted with EtOAc $(2 \times 60 \mathrm{~mL})$. The combined organic layers were washed with $\mathrm{NaHCO}_{3}$ $(5 \%, 70 \mathrm{~mL})$ and brine $(100 \mathrm{~mL})$, and dried $\left(\mathrm{MgSO}_{4}\right)$. The solvent was removed in vacuo and the crude product was purified by column chromatography.

\subsubsection{2,4-Diacetamido-2',5'-dihydroxydiphenylether 10a}

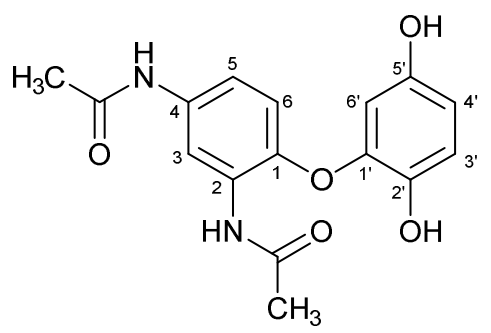

Prepared from 2,4-dinitro-2',5'-dihydroxydiphenylether 7a $(0.57 \mathrm{~g}, 1.955 \mathrm{mmol})$. Elution with EtOAc (100\%) yielded 2,4-diacetamido-2',5'-dihydroxydiphenylether 10a $(0.49 \mathrm{~g}, 1.555 \mathrm{mmol}$, $79.5 \%)$ as a white solid. Recrystallization was achieved from petroleum ether $\left(60-80^{\circ} \mathrm{C}\right)$ : EtOAc; mp 194.5-196 ${ }^{\circ} \mathrm{C}$; (found: $\mathrm{C}, 60.5 ; \mathrm{H}, 5.1 ; \mathrm{N}, 8.65 \% . \mathrm{C}_{16} \mathrm{H}_{16} \mathrm{~N}_{2} \mathrm{O}_{5}$ requires $\mathrm{C}, 60.75$; H, 5.1; N, 8.9\%); m/z $314.9(M-H) ; v_{\max } / \mathrm{cm}^{-1} 3416(\mathrm{~N}-\mathrm{H}), 3317$ (N-H), (br., O-H), 1698 $(\mathrm{C}=\mathrm{O}), 1665(\mathrm{C}=\mathrm{O}), 1616(\mathrm{C}=\mathrm{C}), 1240$ and $1205(\mathrm{C}-\mathrm{O}) ; \delta_{H}\left(300 \mathrm{MHz}, \mathrm{d}_{6}-\mathrm{DMSO}\right) 2.025(3 \mathrm{H}$, $\left.\mathrm{s}, \mathrm{COCH}_{3}, 4-\mathrm{Ac}\right), 2.11\left(3 \mathrm{H}, \mathrm{s}, \mathrm{COCH}_{3}, 2-\mathrm{Ac}\right), 6.28(1 \mathrm{H}, \mathrm{d}, J=2.7 \mathrm{~Hz}, 6-\mathrm{H}), 6.45(1 \mathrm{H}, \mathrm{dd}, J=$ $\left.8.7,2.7 \mathrm{~Hz}, 4^{\prime}-\mathrm{H}\right), 6.70(1 \mathrm{H}, \mathrm{d}, J=8.7 \mathrm{~Hz}, 6-\mathrm{H}), 6.78\left(1 \mathrm{H}, \mathrm{d}, J=8.7 \mathrm{~Hz}, 3^{\prime}-\mathrm{H}\right), 7.35$ (1H, dd, $J$ $=9.0,2.4 \mathrm{~Hz}, 5-\mathrm{H}), 8.12(1 \mathrm{H}, \mathrm{d}, J=2.1 \mathrm{~Hz}, 3-\mathrm{H}), 8.67\left(1 \mathrm{H}, \mathrm{s}, 2^{\prime}-\mathrm{OH}\right), 8.88\left(1 \mathrm{H}, \mathrm{s}, 5^{\prime}-\mathrm{OH}\right)$, $9.44(1 \mathrm{H}, \mathrm{s}, 2-\mathrm{N} H), 9.89(1 \mathrm{H}, \mathrm{s}, 4-\mathrm{N} H) ; \delta_{C}\left(75 \mathrm{MHz}, \mathrm{d}_{6}-\mathrm{DMSO}\right) 24.2\left(\mathrm{CH}_{3}, 2-\mathrm{Ac}\right.$ or 4-Ac), $24.3\left(\mathrm{CH}_{3}, 2-\mathrm{Ac}\right.$ or 4-Ac), $108.3(\mathrm{CH}, 6 '-\mathrm{C}), 111.85(\mathrm{CH}, 4 '-\mathrm{C}), 114.9(\mathrm{CH}, 3-\mathrm{C}), 116.1(\mathrm{CH}$, 5-C), 117.0 (CH, 6-C), 118.0 (CH, 3'-C), 128.85 (quat., 4-C), 134.6 (quat., 2-C), 141.3 (quat., 2'-C), 144.0 (quat., 1-C and 1'-C), 150.8 (quat., 5'-C), 168.5 (quat., 4-Ac, C=O), 169.1 (quat., 2-Ac, $\mathrm{C}=\mathrm{O}$ ). 


\subsubsection{2,4-Diacetamido-3',4'-dimethyl-2',5'-benzoquinone 12a}

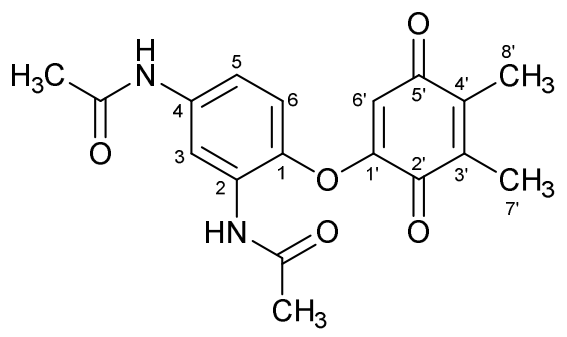

Prepared from 2,4-dinitrophenoxy-3',4'-dimethyl-2',5'-benzoquinone 8b $(0.26 \mathrm{~g}, 0.88 \mathrm{mmol})$ and acetic anhydride $(2.5 \mathrm{~mL})$. Elution with a gradient mixture of petroleum ether $\left(60-80^{\circ} \mathrm{C}\right)$ : EtOAc (10:90 to 0:100) yielded 2,4-diacetamido-3',4'-dimethyl-2',5'-benzoquinone 12a as a bright orange crystalline solid $(0.26 \mathrm{~g}, 0.77 \mathrm{mmol}, 95.8 \%)$. Recrystallization from EtOAc : hexane produced small orange prisms, $\mathrm{mp} 161^{\circ} \mathrm{C}$ (dec.); (found: $\mathrm{C}, 63.4 ; \mathrm{H}, 5.4 ; \mathrm{N}, 8.1 \%$. $\mathrm{C}_{18} \mathrm{H}_{18} \mathrm{~N}_{2} \mathrm{O}_{5}$ requires $\left.\mathrm{C}, 63.15 ; \mathrm{H}, 5.3 ; \mathrm{N}, 8.2 \%\right) ; \mathrm{m} / \mathrm{Z} 298.9\left(M-\mathrm{CH}_{3} \mathrm{CO}\right)^{-}, 340.9(M-H)^{-}$; $v_{\max } / \mathrm{cm}^{-1} 3403$ and $3277(\mathrm{~N}-\mathrm{H}), 1711(\mathrm{C}=\mathrm{O}), 1655$ and $1648(\mathrm{C}=\mathrm{O}$, quinone), $1606(\mathrm{C}=\mathrm{C})$, 1207 (C-O); $\delta_{H}\left(300 \mathrm{MHz}, \mathrm{d}_{8}-\mathrm{THF}\right) 2.00\left(3 \mathrm{H}, \mathrm{d}, J=1.2 \mathrm{~Hz}, 7^{\prime}-\mathrm{H}\right), 2.05-2.06\left(6 \mathrm{H}, \mathrm{m}, 2 \times \mathrm{CH}_{3}\right.$, 8'-H and 2-Ac or 4-Ac), 2.08 (3H, s, 2-Ac or 4-Ac), $5.655\left(1 \mathrm{H}, \mathrm{s}, 66^{\prime}-\mathrm{H}\right), 6.98(1 \mathrm{H}, \mathrm{d}, J=$ $9.0 \mathrm{~Hz}, 6-\mathrm{H}), 7.915(1 \mathrm{H}, \mathrm{dd}, J=8.7,2.1 \mathrm{~Hz}, 5-\mathrm{H}), 8.30(1 \mathrm{H}, \mathrm{d}, J=2.4 \mathrm{~Hz}, 3-\mathrm{H}), 8.63(1 \mathrm{H}, \mathrm{s}, 2-$ $\mathrm{NH}), 9.31(1 \mathrm{H}, \mathrm{s}, 4-\mathrm{N} H)$; $\delta_{C}\left(75 \mathrm{MHz}, \mathrm{d}_{8}\right.$-THF) $9.1\left(\mathrm{CH}_{3}, 7^{\prime}-\mathrm{C}\right.$ or 8'-C), $9.3\left(\mathrm{CH}_{3}, 7^{\prime}-\mathrm{C}\right.$ or 8'C), $21.1\left(\mathrm{CH}_{3}, 2-\mathrm{Ac}\right.$ or 4-Ac), $21.25\left(\mathrm{CH}_{3}, 2-\mathrm{Ac}\right.$ or $\left.4-\mathrm{Ac}\right), 108.5(\mathrm{CH}, 6$ '-C), $110.45(\mathrm{CH}, 3-$ C), 113.0 (CH, 5-C), 118.8 (CH, 6-C), 129.5 (quat., 2-C), 135.35 (quat., 4-C), 136.2 (quat., 1 C), 136.6 (quat., 3'-C), 138.8 (quat., 4'-C), 156.1 (quat., 1'-C), 165.8 (quat., 2-Ac or 4-Ac, $\mathrm{C}=\mathrm{O}$ ), 166.3 (quat., 2-Ac or 4-Ac, $\mathrm{C}=\mathrm{O}$ ), 179.0 (quat., 2'-C), 184.3 (quat., 5'-C).

\subsubsection{2,4-Diacetamido-4'-'butyl-2',5'-benzoquinone 12b}

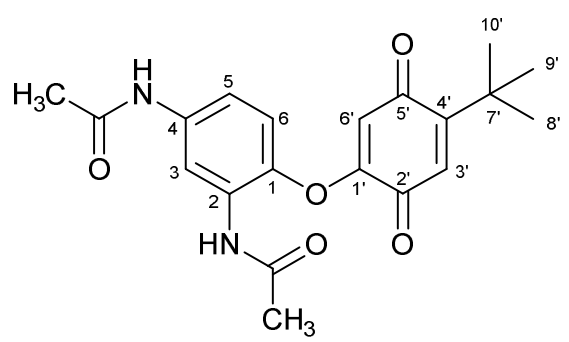

Prepared from 2,4-dinitrophenoxy-4'-'butyl-2',5'-benzoquinone 8c $(0.18 \mathrm{~g}, 0.51 \mathrm{mmol})$. Elution with petroleum ether $\left(60-80^{\circ}\right)$ : EtOAc $(20$ : 80) yielded 2,4-diacetamido-4'-'butyl-2',5'benzoquinone $12 \mathrm{~b}$ as a yellow-brown solid $(0.19 \mathrm{~g}, 0.51 \mathrm{mmol}, 99.0 \%)$. Recrystallization from petroleum ether $\left(60-80^{\circ} \mathrm{C}\right)$ : EtOAc produced light brown micro-crystalline solid; mp $174-$ $178^{\circ} \mathrm{C}$; (found: $\mathrm{C}, 64.9 ; \mathrm{H}, 6.1 ; \mathrm{N}, 7.5 \% . \mathrm{C}_{20} \mathrm{H}_{22} \mathrm{~N}_{2} \mathrm{O}_{5}$ requires $\mathrm{C}, 64.85 ; \mathrm{H}, 6.0 ; \mathrm{N}, 7.6 \%$ ); $\mathrm{m} / \mathrm{Z}$ 
$371.2(M H)^{+} ; v_{\max } / \mathrm{cm}^{-1} 3330(\mathrm{~N}-\mathrm{H}), 2997-2868(\mathrm{C}-\mathrm{H}), 1697(\mathrm{C}=\mathrm{O}), 1665$ and $1639(\mathrm{C}=\mathrm{O}$, quinone), 1594 and $1534(\mathrm{C}=\mathrm{C}), 1168(\mathrm{C}-\mathrm{O})$; $\delta_{H}\left(300 \mathrm{MHz}, \mathrm{d}_{6}\right.$-DMSO) $1.25(9 \mathrm{H}, \mathrm{s}$, $\left.\mathrm{C}\left(\mathrm{CH}_{3}\right)_{3}\right), 2.03(3 \mathrm{H}, \mathrm{s}, 2-\mathrm{Ac}$ or $4-\mathrm{Ac}), 2.055(3 \mathrm{H}, \mathrm{s}, 2-\mathrm{Ac}$ or $4-\mathrm{Ac}), 5.42\left(1 \mathrm{H}, \mathrm{s}, 6{ }^{\prime}-\mathrm{H}\right), 6.63$ $(1 \mathrm{H}, \mathrm{s}, 3 \mathrm{\prime}-\mathrm{H}), 7.13(1 \mathrm{H}, \mathrm{d}, J=9.0 \mathrm{~Hz}, 6-\mathrm{H}), 7.55(1 \mathrm{H}, \mathrm{dd}, J=9.0,2.4 \mathrm{~Hz}, 5-\mathrm{H}), 8.18(1 \mathrm{H}, \mathrm{d}, J$ $=2.4 \mathrm{~Hz}, 3-\mathrm{H}), 9.33(1 \mathrm{H}, \mathrm{s}, 2-\mathrm{NH}), 10.07(1 \mathrm{H}, \mathrm{s}, 4-\mathrm{NH}) ; \delta_{C}\left(75 \mathrm{MHz}, \mathrm{d}_{6}-\mathrm{DMSO}\right) 24.1\left(\mathrm{CH}_{3}\right.$, 2-Ac or 4-Ac), $24.4\left(\mathrm{CH}_{3}, 2-\mathrm{Ac}\right.$ or $\left.4-\mathrm{Ac}\right), 29.5\left(\mathrm{CH}_{3}, 8^{\prime} / 9^{\prime} / 10^{\prime}-\mathrm{C}\left(\mathrm{CH}_{3}\right)_{3}\right), 35.3$ (quat., 7'$\left.\mathrm{C}\left(\mathrm{CH}_{3}\right)_{3}\right), 112.2\left(\mathrm{CH}, 6{ }^{\prime}-\mathrm{C}\right), 115.2(\mathrm{CH}, 3-\mathrm{C}), 116.2(\mathrm{CH}, 5-\mathrm{C}), 121.9(\mathrm{CH}, 6-\mathrm{C}), 130.4(\mathrm{CH}$, 3'-C), 130.9 (quat., 2-C), 137.9 (quat., 1-C), 138.5 (quat., 4-C), 155.7 (quat., 4'-C), 157.6 (quat., 1'-C), 168.8 (quat., 2-Ac or 4-Ac, $\mathrm{C}=\mathrm{O}$ ), 169.2 (quat., 2-Ac or 4-Ac, $\mathrm{C}=\mathrm{O}$ ), 182.1 (quat., 2'-C), 187.8 (quat., 5'-C).

\subsubsection{2,4-Diacetamido-4'-pentyl-2',5'-benzoquinone $12 \mathrm{c}$}

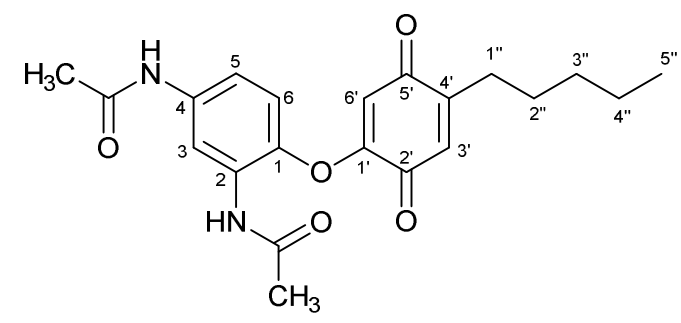

Prepared from 2,4-dinitrophenoxy-4'-pentyl-2',5'-benzoquinone 8d (0.46g, 1.26mmol). The crude product was purified by column chromatography on silica eluting with petroleum ether $\left(60-80^{\circ} \mathrm{C}\right)$ : EtOAc (90:10). 2,4-Diacetamido-4'-pentyl-2',5'-benzoquinone 12c was isolated as a dark orange crystalline solid $(0.48 \mathrm{~g}, 1.24 \mathrm{mmol}, 98.3 \%)$. Recrystallization from EtOAc : hexane produced red prisms; mp $90-93^{\circ} \mathrm{C}$; (found: $\mathrm{C}$, 65.6; $\mathrm{H}, 6.4 ; \mathrm{N}, 7.2 \% . \mathrm{C}_{21} \mathrm{H}_{24} \mathrm{~N}_{2} \mathrm{O}_{5}$ requires $\mathrm{C}, 65.6 ; \mathrm{H}, 6.3 ; \mathrm{N}, 7.3 \%) ; \mathrm{m} / \mathrm{z} 341.0\left(M-\mathrm{CH}_{3} \mathrm{CO}\right)^{-}, 383.0(M-H)^{-} ; v_{\max } / \mathrm{cm}^{-1} 3357(\mathrm{~N}-$ H), $3269(\mathrm{~N}-\mathrm{H}), 2955(\mathrm{C}-\mathrm{H}), 2929(\mathrm{C}-\mathrm{H}), 1726(\mathrm{C}=\mathrm{O}), 1663$ and 1647 (C=O, quinone), 1599 $(\mathrm{C}=\mathrm{C}), 1206(\mathrm{C}-\mathrm{O}) ; \delta_{H}\left(300 \mathrm{MHz}, \mathrm{d}_{8}\right.$-THF) 0.93 (3H, t, $\left.J=6.9 \mathrm{~Hz}, 5 "-\mathrm{H}\right), 1.34-1.39$ (4H, m, 3"-H and 4"-H), 1.48-1.55 (2H, m, 2"-H), 2.04 (3H, s, 2-Ac or 4-Ac), 2.07 (3H, s, 2-Ac or 4Ac), $2.40(2 \mathrm{H}, \mathrm{dt}, J=7.5,1.2 \mathrm{~Hz}, 1 "-\mathrm{H}), 5.67(1 \mathrm{H}, \mathrm{s}, 6$ '-H), 6.59 (1H, br. s, 3'-H), $6.98(1 \mathrm{H}, \mathrm{d}$, $J=8.7 \mathrm{~Hz}, 6-\mathrm{H}), 7.91(1 \mathrm{H}, \mathrm{dd}, J=9.0,2.1 \mathrm{~Hz}, 5-\mathrm{H}), 8.28(1 \mathrm{H}, \mathrm{d}, J=2.1 \mathrm{~Hz}, 3-\mathrm{H}), 8.63(1 \mathrm{H}, \mathrm{s}$, 2-NH), $9.31(1 \mathrm{H}, \mathrm{s}, 4-\mathrm{N} H) ; \delta_{C}\left(75 \mathrm{MHz}, \mathrm{d}_{8}-\mathrm{THF}\right) 13.3\left(\mathrm{CH}_{3}, 5\right.$ "-C), $22.3\left(\mathrm{CH}_{2}, 3\right.$ "-C or 4"-C), $23.0\left(\mathrm{CH}_{3}, 2\right.$-Ac or 4-Ac), $23.1\left(\mathrm{CH}_{3}, 2\right.$-Ac or 4-Ac), $27.85\left(\mathrm{CH}_{2}, 2 "-\mathrm{C}\right), 28.6\left(\mathrm{CH}_{2}, 1 "-\mathrm{C}\right)$, $31.5\left(\mathrm{CH}_{2}, 3\right.$ "-C or 4"-C), $110.8(\mathrm{CH}, 6$ '-C), $112.5(\mathrm{CH}, 3-\mathrm{C}), 114.9(\mathrm{CH}, 5-\mathrm{C}), 120.7$ (CH, 6C), 130.45 (CH, 3'-C), 131.3 (quat., 2-C), 137.15 (quat., 1-C), 138.2 (quat., 4-C), 149.8 (quat., 
4'-C), 158.2 (quat., 1'-C), 167.7 (quat., 2-Ac or 4-Ac, $\mathrm{C}=\mathrm{O}$ ), 168.1 (quat.,2-Ac or 4-Ac, $\mathrm{C}=\mathrm{O}$ ), 181.0 (quat., 2'-C), 186.6 (quat., 5'-C).

\subsubsection{2,5-Diacetamido-2',5'-dihydroxy-4'- 'butyldiphenylether 18a}

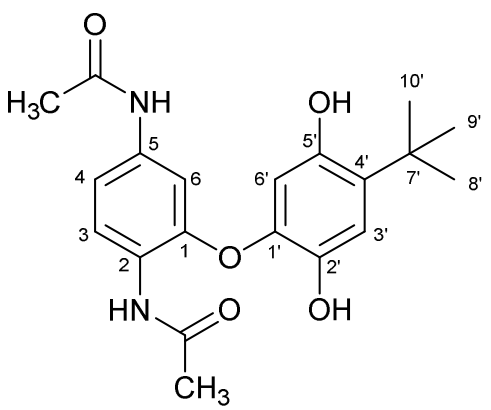

Prepared from 2,5-dinitrophenoxy-4'-'butyl-2',5'-benzoquinone 15a $(0.11 \mathrm{~g}, \quad 0.32 \mathrm{mmol})$. Elution with petroleum ether $\left(60-80^{\circ} \mathrm{C}\right)$ : EtOAc (20:80) yielded 2,5-diacetamido-2',5'dihydroxy-4'- ${ }^{t}$ butyldiphenylether $\mathbf{1 8 a}(0.12 \mathrm{~g}, 0.31 \mathrm{mmol}, 97.5 \%)$ was isolated as a white solid. Recrystallization from petroleum ether $\left(60-80^{\circ} \mathrm{C}\right)$ : EtOAc produced a white crystalline solid; mp 148-152 ${ }^{\circ} \mathrm{C}$; (found: $\mathrm{C}, 64.7 ; \mathrm{H}, 6.55 ; \mathrm{N}, 7.6 \% . \mathrm{C}_{20} \mathrm{H}_{24} \mathrm{~N}_{2} \mathrm{O}_{5}$ requires $\mathrm{C}, 64.5 ; \mathrm{H}, 6.5 ; \mathrm{N}$, $7.5 \%) ; \mathrm{m} / \mathrm{z} 371.0(M-H)^{-} ; v_{\max } / \mathrm{cm}^{-1} 3345(\mathrm{~N}-\mathrm{H}), 3259$ (N-H), (br., O-H), 2957 (C-H), 1701 $(\mathrm{C}=\mathrm{O}), 1667(\mathrm{C}=\mathrm{O}), 1605(\mathrm{C}=\mathrm{C}) ; \delta_{H}\left(300 \mathrm{MHz}, \mathrm{d}_{6}-\mathrm{DMSO}\right) 1.25\left(9 \mathrm{H}, \mathrm{s}, \mathrm{C}\left(\mathrm{CH}_{3}\right)_{3}\right), 1.87(3 \mathrm{H}$, s, 2-Ac or 5-Ac), 1.99 (3H, s, 2-Ac or 5-Ac), $6.36(1 \mathrm{H}, \mathrm{s}, 3$ '-H), $6.69(1 \mathrm{H}, \mathrm{s}, 6$ '-H), $6.85(1 \mathrm{H}$, $\mathrm{d}, J=1.8 \mathrm{~Hz}, 6-\mathrm{H}), 7.25(1 \mathrm{H}, \mathrm{dd}, J=8.4,1.5 \mathrm{~Hz}, 4-\mathrm{H}), 7.60(1 \mathrm{H}, \mathrm{d}, J=8.7 \mathrm{~Hz}, 3-\mathrm{H}), 8.44(1 \mathrm{H}$, s, 2'-OH), $8.76\left(1 \mathrm{H}, \mathrm{s}, 55^{\prime}-\mathrm{OH}\right), 9.33(1 \mathrm{H}, \mathrm{s}, 2-\mathrm{NH}), 9.77(1 \mathrm{H}, \mathrm{s}, 5-\mathrm{N} H) ; \delta_{C}\left(75 \mathrm{MHz}, \mathrm{d}_{6^{-}}\right.$ DMSO) $24.0\left(\mathrm{CH}_{3}, 2-\mathrm{Ac}\right.$ or 5-Ac), $24.3\left(\mathrm{CH}_{3}, 2-\mathrm{Ac}\right.$ or 5-Ac), $29.9\left(\mathrm{CH}_{3}, 8^{\prime} / 9^{\prime} / 10^{\prime}-\mathrm{C}\left(\mathrm{CH}_{3}\right)_{3}\right)$, 34.5 (quat., 7'- $\left.C\left(\mathrm{CH}_{3}\right)_{3}\right), 107.1(\mathrm{CH}, 6-\mathrm{C}), 109.7(\mathrm{CH}, 3 '-\mathrm{C}), 113.1(\mathrm{CH}, 4-\mathrm{C}), 115.9(\mathrm{CH}, 6$ 'C), 123.55 (quat., 2-C), 124.2 (CH, 3-C), 132.8 (quat., 4'-C), 136.9 (quat., 5-C), 140.4 (quat., 1'-C), 140.7 (quat., 2'-C), 148.8 (quat., 5'-C), 149.7 (quat., 1-C), 168.5 (quat., 2-Ac or 5-Ac, $\mathrm{C}=\mathrm{O}$ ), 169.0 (quat., 2-Ac or 5-Ac, $\mathrm{C}=\mathrm{O}$ ).

\subsection{Synthesis of 8-amino-alkyl-3H-phenoxazin-3-ones 2a-f and 7-amino-2-alkyl-3H- phenoxazin-3-ones 17a-b}

\subsubsection{General procedure}

The appropriately substituted 2,4-dinitro-2',5'-dihydroxydiphenyl ether 7a-b was dissolved in $\mathrm{MeOH}(10 \mathrm{~mL} / \mathrm{mmol}$ of 2,4-dinitro-2',5'-dihydroxydiphenyl ether), or the appropriately substituted dinitrophenoxy-2',5'-benzoquinone 8a-e or 15a-b was dissolved in an EtOAc : $\mathrm{MeOH}(1: 1)$ solvent mixture $(20 \mathrm{~mL} / \mathrm{mmol}$ of dinitrophenoxy-2',5'-benzoquinone). $\mathrm{Pd} / \mathrm{C} 5 \%$ 
$(10 \% \mathrm{w} / \mathrm{w}$ of starting material) was added to the solution and the resulting mixture was hydrogenated in a Berghof apparatus. The $\mathrm{H}_{2}$ pressure was set to $2.4 \mathrm{bar}$ and reset to this pressure when necessary. When the $\mathrm{H}_{2}$ pressure was steady, the catalyst was removed by filtration through Celite, which was washed several times with small portions of $\mathrm{MeOH}$. Either $\mathrm{AgO}, \mathrm{Ag}_{2} \mathrm{O}$ or $\mathrm{MnO}_{2}$ (3mol equivalent) was then added quickly to the filtrate; when reducing 2',5'-dihydroxydiphenyl ether $\mathbf{7 a - b}, \mathrm{Et}_{3} \mathrm{~N}(1 \mathrm{~mL})$ was also added. A purple suspension was rapidly produced for 8-amino-alkyl-3H-phenoxazin-3-ones $2 \mathbf{a}-\mathbf{f}$ and a redpink fluorescent suspension for 7-amino-alkyl-3H-phenoxazin-3-ones 17a-b. The filtrate was then stirred at $\mathrm{rt}$ and completion of the reaction was monitored by TLC. The metal oxide was then removed by filtration through Celite, which was washed several times with $\mathrm{MeOH}$. The combined filtrates were evaporated in vacuo and the residue subjected to column chromatography on silica.

\subsubsection{8-Amino-3H-phenoxazin-3-one 2 a}

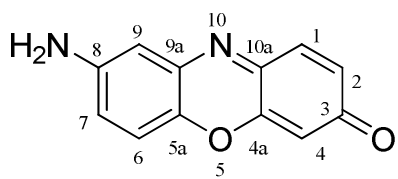

Prepared from 2,4-dinitro-2'-5'-dihydroxydiphenylether $7 \mathbf{a}(0.56 \mathrm{~g}, 1.91 \mathrm{mmol})$. Elution with a gradient mixture of petroleum ether $\left(60-80^{\circ} \mathrm{C}\right)$ : EtOAc (70:30 to 100:0), yielded 8-amino$3 H$-phenoxazin-3-one 2a as a dark purple solid $(0.37 \mathrm{~g}, 1.75 \mathrm{mmol}, 91.3 \%)$; $\mathrm{mp}>290^{\circ} \mathrm{C}$; (found: $\mathrm{C}, 68.2 ; \mathrm{H}, 3.9 ; \mathrm{N}, 13.4 \% . \mathrm{C}_{12} \mathrm{H}_{8} \mathrm{~N}_{2} \mathrm{O}_{2}$ requires $\mathrm{C}, 67.9 ; \mathrm{H}, 3.8 ; \mathrm{N}, 13.2 \%$ ); $/ \mathrm{z} 213.0$ $(M H)^{+}$and $234.9(M N a)^{+} ; v_{\max } / \mathrm{cm}^{-1} 3387,3320,3200\left(\mathrm{NH}_{2}\right), 1640(\mathrm{C}=\mathrm{O}), 1591(\mathrm{C}=\mathrm{C}) ; \delta_{\mathrm{H}}$ (300 MHz, d 6 -DMSO) $5.45\left(2 \mathrm{H}\right.$, br. s, $\left.\mathrm{NH}_{2}\right), 6.21(1 \mathrm{H}, \mathrm{d}, J=2.1 \mathrm{~Hz}, 4-\mathrm{H}), 6.82(1 \mathrm{H}, \mathrm{dd}, J=$ 9.6, 2.1Hz, 2-H), $6.97(2 \mathrm{H}, \mathrm{m}, 7-\mathrm{H}$ and 9-H), $7.24(1 \mathrm{H}, \mathrm{d}, J=9.6 \mathrm{~Hz}, 6-\mathrm{H}), 7.49(1 \mathrm{H}, \mathrm{d}, J=$ 9.9Hz, 1-H); $\delta_{\mathrm{C}}\left(75 \mathrm{MHz}, \mathrm{d}_{6}-\mathrm{DMSO}\right) 105.3(\mathrm{CH}, 4-\mathrm{C}), 111.6(\mathrm{CH}, 9-\mathrm{C}), 116.7(\mathrm{CH}, 6-\mathrm{C})$, $121.0(\mathrm{CH}, 7-\mathrm{C}), 134.3$ (quat., 8-C), $135.0(\mathrm{CH}, 1-\mathrm{C}$ and 2-C), 135.5 (quat., 9a-C), 147.4 (quat., 5a-C), 148.2 (quat., 10a-C), 150.4 (quat., 4a-C), 185.6 (quat., 3-C).

\subsubsection{8-Amino-1,2,4-trimethyl-3H-phenoxazin-3-one $2 \mathrm{~b}$}

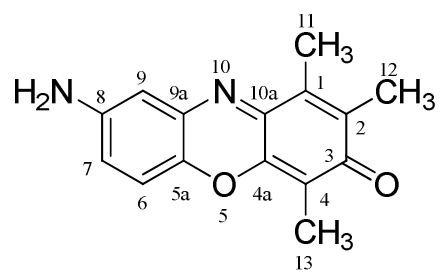


Prepared from 2,4-dinitrophenoxy-3',4',6'-trimethylbenzoquinone 8a $(0.27 \mathrm{~g}, 0.81 \mathrm{mmol})$. Elution using petroleum ether $\left(60-80^{\circ} \mathrm{C}\right)$ : EtOAc (30:70) yielded 8-amino-1,2,4-trimethyl$3 H$-phenoxazin-3-one $\mathbf{2 b}$ as a dark violet solid $(0.19 \mathrm{~g}, 0.77 \mathrm{mmol}, 94.0 \%)$. Analytical data were identical to the product obtained from 2,4-dinitro-2',5'-dihydroxy-3',4',6'trimethyldiphenylether $7 \mathbf{b}$ below.

Also prepared from 2,4-dinitro-2',5'-dihydroxy-3',4',6'-trimethyldiphenylether $7 \mathbf{b} \quad(0.33 \mathrm{~g}$, $0.98 \mathrm{mmol})$. Elution using petroleum ether $\left(60-80^{\circ} \mathrm{C}\right)$ : EtOAc $(30: 70)$ yielded 8-amino-1,2,4trimethyl-3H-phenoxazin-3-one $\mathbf{2 b}$ as a dark violet solid $(0.22 \mathrm{~g}, 0.85 \mathrm{mmol}, 86.7 \%)$; $\mathrm{mp} 235$ $246^{\circ} \mathrm{C} ; \mathrm{m} / \mathrm{Z} 255.0(\mathrm{MH})^{+} ; v_{\max } / \mathrm{cm}^{-1} 3355,3332$ and $3219\left(\mathrm{NH}_{2}\right), 2850-2920(\mathrm{C}-\mathrm{H}), 1649$ $(\mathrm{C}=\mathrm{O}), 1595,1577$ and $1505(\mathrm{C}=\mathrm{C}) ; \delta_{\mathrm{H}}\left(300 \mathrm{MHz}, \mathrm{d}_{6}\right.$-DMSO) $1.95(3 \mathrm{H}, \mathrm{s}, 13-\mathrm{H}), 2.06(3 \mathrm{H}$, $\mathrm{d}, J=0.9 \mathrm{~Hz}, 12-\mathrm{H}), 2.32(3 \mathrm{H}, \mathrm{d}, J=0.9 \mathrm{~Hz}, 11-\mathrm{H}), 5.32\left(2 \mathrm{H}\right.$, br. s, $\left.\mathrm{NH} H_{2}\right), 6.86(1 \mathrm{H}, \mathrm{dd}, J=$ $8.7,2.7 \mathrm{~Hz}, 7-\mathrm{H}), 6.93(1 \mathrm{H}, \mathrm{d}, J=2.7 \mathrm{~Hz}, 9-\mathrm{H}), 7.19(1 \mathrm{H}, \mathrm{d}, J=8.7 \mathrm{~Hz}, 6-\mathrm{H}) ; \delta_{\mathrm{C}}\left(75 \mathrm{MHz}, \mathrm{d}_{6}-\right.$ DMSO) $8.08\left(\mathrm{CH}_{3}, 13-\mathrm{C}\right), 13.0\left(\mathrm{CH}_{3}, 11-\mathrm{C}\right), 13.4\left(\mathrm{CH}_{3}, 12-\mathrm{C}\right), 111.75$ (quat., 4-C), 112.1 (CH, 9-C), 116.3 (CH, 6-C), 119.6 (CH, 7-C), 133.3 (quat., 8-C), 135.8 (quat., 9a-C), 136.6 (quat., 1-C or 2-C), 138.4 (quat., 1-C or 2-C), 145.9 (quat., 4a-C), 146.8 (quat., 5a-C), 148.1 (quat., 10a-C), 184.1 (quat.,3-C). HPLC-MS spectra: Figure S1.

\subsubsection{8-Amino-1,2-dimethyl-3H-phenoxazin-3-one 2c}

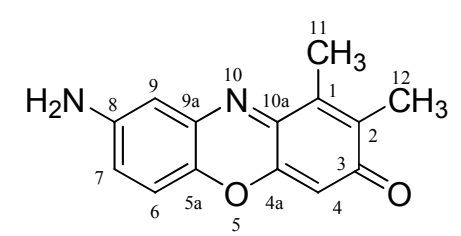

Prepared from 2,4-dinitrophenoxy-3',4'-dimethyl-2',5'-benzoquinone $\mathbf{8 b}(0.41 \mathrm{~g}, 1.28 \mathrm{mmol})$. Elution with petroleum ether $\left(60-80^{\circ} \mathrm{C}\right)$ : EtOAc yielded 8-amino-1,2-dimethyl-3Hphenoxazin-3-one $2 \mathrm{c}(0.28 \mathrm{~g}, 1.18 \mathrm{mmol}, 92.0 \%)$ as a dark violet solid; $\mathrm{mp} 260-265^{\circ} \mathrm{C} ; \mathrm{m} / \mathrm{Z}$ $241.0(\mathrm{MH})^{+} ; v_{\max } / \mathrm{cm}^{-1} 3414,3332$ and $3225\left(\mathrm{NH}_{2}\right), 2851-2920(\mathrm{C}-\mathrm{H}), 1638(\mathrm{C}=\mathrm{O}), 1570$ $(\mathrm{C}=\mathrm{C}) ; \delta_{\mathrm{H}}\left(300 \mathrm{MHz}, \mathrm{d}_{6}-\mathrm{DMSO}\right) 2.05(3 \mathrm{H}, \mathrm{d}, J=0.9 \mathrm{~Hz}, 12-\mathrm{H}), 2.35(3 \mathrm{H}, \mathrm{d}, J=0.9 \mathrm{~Hz}, 11-$ H), $5.38\left(2 \mathrm{H}\right.$, br. s, $\left.\mathrm{NH}_{2}\right), 6.12(1 \mathrm{H}, \mathrm{s}, 4-\mathrm{H}), 6.90(1 \mathrm{H}, \mathrm{dd}, J=9.0,2.7 \mathrm{~Hz}, 7-\mathrm{H}), 6.96(1 \mathrm{H}, \mathrm{d}, J$ $=2.7 \mathrm{~Hz}, 9-\mathrm{H}), 7.20(1 \mathrm{H}, \mathrm{d}, J=8.7 \mathrm{~Hz}, 6-\mathrm{H}) ; \delta_{\mathrm{C}}\left(75 \mathrm{MHz}, \mathrm{d}_{6}-\mathrm{DMSO}\right) 13.1\left(\mathrm{CH}_{3}, 11-\mathrm{C}\right.$ or $12-$ C), $13.2\left(\mathrm{CH}_{3}, 11-\mathrm{C}\right.$ or 12-C), $104.0(\mathrm{CH}, 4-\mathrm{C}), 112.0(\mathrm{CH}, 9-\mathrm{C}), 116.3(\mathrm{CH}, 6-\mathrm{C}), 120.1$ (CH, 7-C), 133.7 (quat., 8-C), 135.3 (quat., 9a-C), 137.5 (quat., 1-C), 139.1 (quat., 2-C), 147.1 (quat., 5a-C), 148.2 (quat., 10a-C), 149.8 (quat., 4a-C), 184.5 (quat., 3-C). HPLC-MS spectra: Figure S2. 


\subsubsection{8-Amino-2- ${ }^{t}$ butyl-3H-phenoxazin-3-one $2 \mathrm{~d}$}

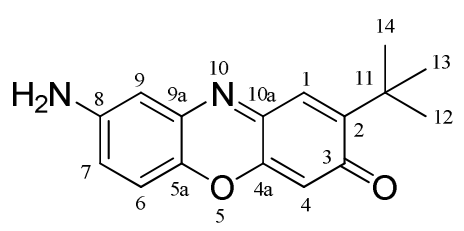

Prepared from 2,4-dinitrophenoxy-4'-'butyl-2',5'-benzoquinone 8c $(0.62 \mathrm{~g}, 1.78 \mathrm{mmol})$. Elution with petroleum ether $\left(60-80^{\circ} \mathrm{C}\right)$ : EtOAc $(40: 60)$ yielded 8 -amino-2- ${ }^{t}$ butyl-3 $H$-phenoxazin-3one $2 \mathrm{~d}(0.41 \mathrm{~g}, 1.55 \mathrm{mmol}, 86.8 \%)$ as a dark violet solid; $\mathrm{mp} 217-226^{\circ} \mathrm{C}$; (found: $\mathrm{C}, 71.6$; $\mathrm{H}$, 6.3; N, 10.1\%. $\mathrm{C}_{16} \mathrm{H}_{16} \mathrm{~N}_{2} \mathrm{O}_{2}$ requires $\left.\mathrm{C}, 71.6 ; \mathrm{H}, 6.0 ; \mathrm{N}, 10.4 \%\right) ; \mathrm{m} / \mathrm{Z} 269.0(M H)^{+} ; v_{\max } / \mathrm{cm}^{-1}$ 3420 and $3337\left(\mathrm{NH}_{2}\right), 2952(\mathrm{C}-\mathrm{H}), 1649(\mathrm{C}=\mathrm{O}), 1601$ and $1575(\mathrm{C}=\mathrm{C}) ; \delta_{\mathrm{H}}\left(300 \mathrm{MHz}, \mathrm{CDCl}_{3}\right)$ $1.31\left(9 \mathrm{H}, \mathrm{s}, \mathrm{C}\left(\mathrm{CH}_{3}\right)_{3}\right), 3.74\left(2 \mathrm{H}\right.$, br. s, $\left.\mathrm{NH}_{2}\right), 6.12(1 \mathrm{H}, \mathrm{s}, 4-\mathrm{H}), 6.78(1 \mathrm{H}, \mathrm{dd}, J=8.7,2.7 \mathrm{~Hz}$, 7-H), $6.94(1 \mathrm{H}, \mathrm{d}, J=2.7 \mathrm{~Hz}, 9-\mathrm{H}), 7.05(1 \mathrm{H}, \mathrm{d}, J=8.7 \mathrm{~Hz}, 6-\mathrm{H}), 7.20(1 \mathrm{H}, \mathrm{s}, 1-\mathrm{H}) ; \delta_{\mathrm{C}}(75$ $\left.\mathrm{MHz}, \mathrm{CDCl}_{3}\right) 29.3\left(\mathrm{CH}_{3}, 12 / 13 / 14-\mathrm{C}\left(\mathrm{CH}_{3}\right)_{3}\right), 35.8$ (quat., 11-C( $\left.\left(\mathrm{CH}_{3}\right)_{3}\right), 107.6(\mathrm{CH}, 4-\mathrm{C})$, 113.6 (CH, 9-C), 116.5 (CH, 6-C), 119.9 (CH, 7-C), 129.4 (CH, 1-C), 134.25 (quat., 8-C), 137.0 (quat., 9a-C), 143.9 (quat., 5a-C), 148.7 (quat., 4a-C or 10a-C), 149.8 (quat., 4a-C or 10a-C), 154.9 (quat., 2-C), 185.9 (quat., 3-C).

\subsubsection{8-Amino-2-pentyl-3H-phenoxazin-3-one $2 \mathrm{e}$}

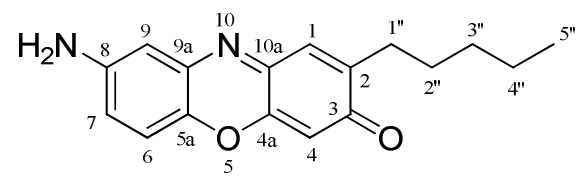

Prepared from 2,4-dinitrophenoxy-4'-pentyl-2',5'-benzoquinone $\mathbf{8 d}(0.38 \mathrm{~g}, 1.07 \mathrm{mmol})$. Elution with a gradient mixture of petroleum ether $\left(60-80^{\circ} \mathrm{C}\right):$ EtOAc $(50: 50$ to $35: 65)$ yielded 8-amino-2-pentyl-3 $H$-phenoxazin-3-one $2 \mathrm{e}$ as a dark violet solid $(0.29 \mathrm{~g}, 1.01 \mathrm{mmol}$, 94.7\%); mp 141-144 ${ }^{\circ} \mathrm{C}$; (found: $\mathrm{C}, 71.9 ; \mathrm{H}, 6.6 ; \mathrm{N}, 9.7 \% . \mathrm{C}_{17} \mathrm{H}_{18} \mathrm{~N}_{2} \mathrm{O}_{2}$ requires $\mathrm{C}, 72.2 ; \mathrm{H}$, 6.4; N, 9.9\%); m/Z $283.1(M H)^{+} ; v_{\max } / \mathrm{cm}^{-1} 3400,3337$ and $3222(\mathrm{~N}-\mathrm{H}), 2853$ and $2928(\mathrm{C}-\mathrm{H})$, $1639(\mathrm{C}=\mathrm{O}), 1585,1566$ and $1508(\mathrm{C}=\mathrm{C}) ; \delta_{\mathrm{H}}\left(300 \mathrm{MHz}, \mathrm{d}_{6}-\mathrm{DMSO}\right) 0.87(3 \mathrm{H}, \mathrm{t}, J=6.9 \mathrm{~Hz}$, 5'-H), 1.28-1.32 (4H, m, 3'-H and 4'-H), 1.46-1.56 (2H, m, 2'-H), 2.45 (2H, t, $\left.J=6.9 \mathrm{~Hz}, 1^{\prime}-\mathrm{H}\right)$, $5.41\left(2 \mathrm{H}\right.$, br. s, $\left.\mathrm{NH}_{2}\right), 6.17(1 \mathrm{H}, \mathrm{s}, 4-\mathrm{H}), 6.90-6.93(2 \mathrm{H}, \mathrm{m}, 7-\mathrm{H}$ and $9-\mathrm{H}), 7.21(1 \mathrm{H}, \mathrm{d}, J=$ 9.3Hz, 6-H), $7.25(1 \mathrm{H}, \mathrm{s}, 1-\mathrm{H}) ; \delta_{\mathrm{C}}\left(75 \mathrm{MHz}, \mathrm{d}_{6}-\mathrm{DMSO}\right) 14.3\left(\mathrm{CH}_{3}, 5^{\prime}-\mathrm{C}\right), 22.4\left(\mathrm{CH}_{2}, 3^{\prime}-\mathrm{C}\right.$ or 4'-C), $28.1\left(\mathrm{CH}_{2}, 2^{\prime}-\mathrm{C}\right), 29.5\left(\mathrm{CH}_{2}, 1^{\prime}-\mathrm{C}\right), 31.5\left(\mathrm{CH}_{2}, 3\right.$ '-C or 4'-C), $105.0(\mathrm{CH}, 4-\mathrm{C}), 111.55$ (CH, 9-C), $116.55(\mathrm{CH}, 6-\mathrm{C}), 120.3(\mathrm{CH}, 7-\mathrm{C}), 130.6(\mathrm{CH}, 1-\mathrm{C}), 134.3$ (quat., 8-C), 135.3 
(quat., 9a-C), 147.25 (quat., 2-C or 5a-C), 147.3 (quat., 2-C or 5a-C), 148.4 (quat., 4a-C), 149.7 (quat., 10a-C), 184.8 (quat., 3-C).

\subsubsection{8-Amino-1-phenyl-3H-phenoxazin-3-one $2 \mathrm{f}$}

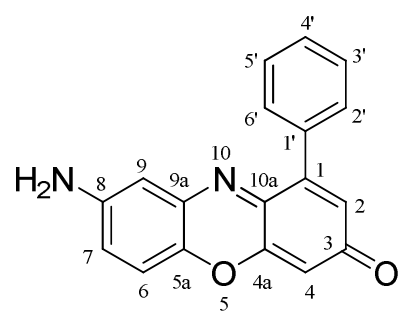

Prepared from 2,4-dinitrophenoxy-3'-phenyl-2',5'-benzoquinone 8e $(0.065 \mathrm{~g}, 0.18 \mathrm{mmol})$ using THF as solvent. Hydrogenation time of $48 \mathrm{~h}$ was required; cyclisation occured in the presence of $\mathrm{Ag}_{2} \mathrm{O}(0.16 \mathrm{~g}, 0.71 \mathrm{mmol})$ and $\mathrm{Et}_{3} \mathrm{~N}(1 \mathrm{~mL})$. Elution with petroleum ether $\left(60-80^{\circ} \mathrm{C}\right): \mathrm{EtOAc}$ (30:70) yielded 8-amino-1-phenyl-3H-phenoxazin-3-one $\mathbf{2 f}$ as a dark violet solid $(0.04 \mathrm{~g}$, 0.13mmol, 73.4\%); mp $205^{\circ} \mathrm{C}$ (dec.); (found: $\mathrm{C}, 75.1 ; \mathrm{H}, 4.4 ; \mathrm{N}, 9.65 \% . \mathrm{C}_{18} \mathrm{H}_{12} \mathrm{~N}_{2} \mathrm{O}_{2}$ requires

C, 75.0; H, 4.2; N, 9.7\%); m/Z $289.0(M H)^{+}, v_{\max } / \mathrm{cm}^{-1} 3421,3308$ and $3198(\mathrm{~N}-\mathrm{H}), 1634$ $(\mathrm{C}=\mathrm{O}), 1586,1560$ and $1502(\mathrm{C}=\mathrm{C}) ; \delta_{\mathrm{H}}\left(300 \mathrm{MHz}, \mathrm{d}_{6}\right.$-DMSO) $5.31\left(2 \mathrm{H}\right.$, br. s, $\left.\mathrm{NH}_{2}\right), 6.18$ $(1 \mathrm{H}, \mathrm{d}, J=2.1 \mathrm{~Hz}, 4-\mathrm{H}), 6.68(1 \mathrm{H}, \mathrm{d}, J=2.1 \mathrm{~Hz}, 2-\mathrm{H}), 6.77(1 \mathrm{H}, \mathrm{d}, J=2.7 \mathrm{~Hz}, 9-\mathrm{H}), 6.87(1 \mathrm{H}$, $\mathrm{dd}, J=8.7,2.7 \mathrm{~Hz}, 7-\mathrm{H}), 7.17(1 \mathrm{H}, \mathrm{d}, J=8.7 \mathrm{~Hz}, 6-\mathrm{H}), 7.38-7.40(3 \mathrm{H}, \mathrm{m}, 3$ '-H, 4'-H and 5'-H), 7.48-7.53 (2H, m, 2'-H and 6'-H); $\delta_{\mathrm{C}}\left(75 \mathrm{MHz}, \mathrm{d}_{6}\right.$-DMSO) $105.15(\mathrm{CH}, 4-\mathrm{C}), 112.0(\mathrm{CH}, 9-$ C), $116.5(\mathrm{CH}, 6-\mathrm{C}), 121.15(\mathrm{CH}, 7-\mathrm{C}), 128.3(\mathrm{CH}, 3 '-\mathrm{C}$ and 5'-C), 129.1 (CH, 4'-C), 130.6 (CH, 2'-C and 6'-C), 133.0 (CH, 2-C), 133.9 (quat., 8-C), 135.3 (quat., 9a-C), 135.5 (quat., 1C), 144.9 (quat., 1'-C), 147.2 (quat., 5a-C or 10a-C), 147.3 (quat., 5a-C or 10a-C), 150.6 (quat., 4a-C), 184.5 (quat., 3-C).

\subsubsection{7-Amino-2- ${ }^{t}$ butyl-3H-phenoxazin-3-one 17 a}

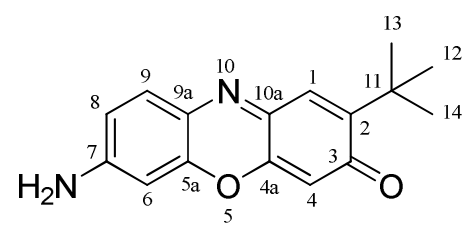

Prepared from 2,5-dinitrophenoxy-4'-'butyl-2',5'-benzoquinone 15a (1.01g, 2.91mmol). Elution with petroleum ether $\left(60-80^{\circ} \mathrm{C}\right)$ : EtOAc (40:60), yielded 7-amino-2-butyl-3Hphenoxazin-3-one 17a as a dark solid with a metallic shine $(0.76 \mathrm{~g}, 2.84 \mathrm{mmol}, 97.5 \%)$; mp 255-260 ${ }^{\circ}$; (found: $\mathrm{C}, 71.5 ; \mathrm{H}, 6.1 ; \mathrm{N}, 10.4 \% . \mathrm{C}_{16} \mathrm{H}_{16} \mathrm{~N}_{2} \mathrm{O}_{2}$ requires $\mathrm{C}, 71.6 ; \mathrm{H}, 6.0 ; \mathrm{N}$, 
10.4\%); $m / z 269.1(M H)^{+} ; v_{\max } / \mathrm{cm}^{-1} 3408,3317$ and $3205\left(\mathrm{NH}_{2}\right), 2944(\mathrm{C}-\mathrm{H}), 1643(\mathrm{C}=\mathrm{O})$, 1589, 1568 and $1547(\mathrm{C}=\mathrm{C}) ; \delta_{\mathrm{H}}\left(300 \mathrm{MHz}, \mathrm{d}_{6}-\mathrm{DMSO}\right) 1.31\left(9 \mathrm{H}, \mathrm{s}, \mathrm{C}(\mathrm{CH})_{3}\right), 6.04(1 \mathrm{H}, \mathrm{s}, 4-$ H), $6.475(1 \mathrm{H}, \mathrm{d}, J=2.4 \mathrm{~Hz}, 6-\mathrm{H}), 6.69(1 \mathrm{H}, \mathrm{dd}, J=9.0,2.4 \mathrm{~Hz}, 8-\mathrm{H}), 6.81\left(2 \mathrm{H}\right.$, br. s, $\left.\mathrm{NH}_{2}\right)$, $7.14(1 \mathrm{H}, \mathrm{s}, 1-\mathrm{H}), 7.43(1 \mathrm{H}, \mathrm{d}, J=8.7 \mathrm{~Hz}, 9-\mathrm{H}) ; \delta_{\mathrm{C}}\left(75 \mathrm{MHz}, \mathrm{d}_{6}\right.$-DMSO) $29.4\left(\mathrm{CH}_{3}, 12 / 13 / 14-\right.$ $\left.\mathrm{C}\left(\mathrm{CH}_{3}\right)_{3}\right), 35.4$ (quat., 11-C( $\left.\left.\mathrm{CH}_{3}\right)_{3}\right), 97.9(\mathrm{CH}, 6-\mathrm{C}), 106.5(\mathrm{CH}, 4-\mathrm{C}), 113.6(\mathrm{CH}, 8-\mathrm{C}), 126.4$ (quat., 7-C), 129.45 (CH, 1-C), 132.0 (CH, 9-C), 140.7 (quat., 10a-C), 146.6 (quat., 9a-C), 149.0 (quat., 4a-C), 150.4 (quat., 2-C), 155.1 (quat., 5a-C), 184.6 (quat., 3-C).

\subsubsection{7-Amino-2-pentyl-3H-phenoxazin-3-one 17b}

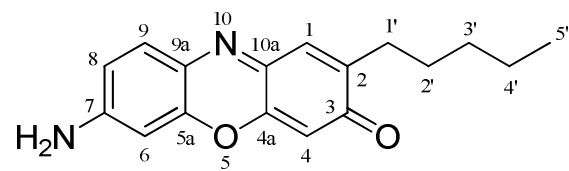

Prepared from 2,5-dinitrophenoxy-4'-pentyl-2',5'-benzoquinone 15b (0.80g, 2.23mmol). Elution with a gradient mixture of petroleum ether $\left(60-80^{\circ} \mathrm{C}\right)$ : EtOAc (40:60 to 0:100) yielded 7-amino-2-pentyl-3H-phenoxazin-3-one $\mathbf{1 7 b}$ as a dark violet solid $(0.62 \mathrm{~g}, 2.18 \mathrm{mmol}$, 97.6\%); mp 250-254 ${ }^{\circ}$; (found: $\mathrm{C}, 72.15 ; \mathrm{H}, 6.6 ; \mathrm{N}, 9.8 \% . \mathrm{C}_{17} \mathrm{H}_{18} \mathrm{~N}_{2} \mathrm{O}_{2}$ requires $\mathrm{C}, 72.2 ; \mathrm{H}$, 6.4; N, 9.9\%); m/Z $283.2(M H)^{+} ; v_{\max } / \mathrm{cm}^{-1} 3420,3329$ and $3226\left(\mathrm{NH}_{2}\right), 2923(\mathrm{C}-\mathrm{H}), 1654$ $(\mathrm{C}=\mathrm{O}), 1599$ and $1565(\mathrm{C}=\mathrm{C})$; $\delta_{\mathrm{H}}\left(300 \mathrm{MHz}, \mathrm{d}_{6}\right.$-DMSO) $0.88\left(3 \mathrm{H}, \mathrm{t}, J=6.9 \mathrm{~Hz}, 5^{\prime}-\mathrm{H}\right), 1.29$ $1.33\left(4 \mathrm{H}, \mathrm{m}, 3^{\prime}-\mathrm{H}\right.$ and 4'-H), 1.47-1.54 (2'-H), $2.45\left(2 \mathrm{H}, \mathrm{t}, J=7.5 \mathrm{~Hz}, 1^{\prime}-\mathrm{H}\right), 6.17(1 \mathrm{H}, \mathrm{s}, 4-\mathrm{H})$, $6.51(1 \mathrm{H}, \mathrm{d}, J=2.4 \mathrm{~Hz}, 6-\mathrm{H}), 6.71(1 \mathrm{H}, \mathrm{dd}, J=8.7,2.4 \mathrm{~Hz}, 8-\mathrm{H}), 6.83\left(2 \mathrm{H}\right.$, br. s, $\left.\mathrm{NH}_{2}\right), 7.24$ $(1 \mathrm{H}, \mathrm{s}, 1-\mathrm{H}), 7.48(1 \mathrm{H}, \mathrm{d}, J=8.7 \mathrm{~Hz}, 9-\mathrm{H}) ; \delta_{\mathrm{C}}\left(75 \mathrm{MHz}, \mathrm{d}_{6}-\mathrm{DMSO}\right) 14.4\left(\mathrm{CH}_{3}, 5^{\prime}-\mathrm{C}\right), 22.4$ $\left(\mathrm{CH}_{2}, 3\right.$ '-C or 4'-C), $28.3\left(\mathrm{CH}_{2}, 2^{\prime}-\mathrm{C}\right), 29.5\left(\mathrm{CH}_{2}, 1^{\prime}-\mathrm{C}\right), 31.55\left(\mathrm{CH}_{2}, 3\right.$ '-C or 4'-C), $97.8(\mathrm{CH}$, 6-C), 105.1 (CH, 4-C), 113.8 (CH, 8-C), 126.4 (quat., 7-C), 130.8 (CH, 1-C), 132.1 (CH, 9C), 140.6 (quat., 9a-C), 144.0 (quat., 4a-C), 146.7 (quat., 10a-C), 149.8 (quat., 2-C), 155.1 (quat., 5a-C), 184.3 (quat., 3-C).

\subsection{Synthesis of $7-N$ and $8-N-\left(N^{\prime}-t^{t}\right.$ butoxycarbonyl- $\beta$-alanyl)amino-3H-phenoxazin-3- ones 20a-b and 21a-f}

\subsubsection{General procedure}

In a $100 \mathrm{~mL}$ flame dried flask flushed with nitrogen, $N$ - ${ }^{t}$ boc- $\beta$-alanine ( 3 mol equivalent) was dissolved in dry THF $\left(10 \mathrm{~mL} / \mathrm{mmol}\right.$ of $N$ - ${ }^{t}$ boc- $\beta$-alanine $)$ and the solution was cooled with an ice bath to $2-3^{\circ} \mathrm{C}\left(\mathrm{T}_{\text {int. }}\right)$. $N$-Methylpiperidine $(3.5 \mathrm{~mol}$ equivalent) was then added dropwise, the resulting solution stirred for $5 \mathrm{~min}$ and isobutylchloroformate (3mol equivalent) was added 
dropwise (temperature rose to $5^{\circ} \mathrm{C}$ ). The resulting cloudy solution was stirred at $3^{\circ} \mathrm{C}$ for $1 \mathrm{~h}$ to allow the mixed anhydride to form.

Method $\boldsymbol{A}$. In the meantime, the correctly substituted 7-amino-3H-phenoxazin-3-one (1mol equivalent) was dissolved in dry DMF ( $5 \mathrm{~mL} / \mathrm{mmol}$ of 7 -amino-3H-phenoxazin-3-one) in a $10 \mathrm{~mL}$ round-bottomed flask, and $\mathrm{Pd} / \mathrm{C} 5 \%(10 \% \mathrm{w} / \mathrm{w}$ of the starting material) was added to the resulting solution. The resulting mixture was hydrogenated in a Berghof apparatus for $1 \mathrm{~h}$ with the $\mathrm{H}_{2}$ pressure initially set at 2 bar. The flask was immediately sealed after hydrogenation and protected with an $\mathrm{N}_{2}$ atmosphere. The resulting 7-amino-3-hydroxyphenoxazine was added via canula to anhydride mixture.

Method B. The correctly substituted 8-amino-3H-phenoxazin-3-one (1mol equivalent) was directly added as a solid to the anhydride mixture.

The resulting mixture (method $\boldsymbol{A}$ or method $\boldsymbol{B}$ ) was stirred under nitrogen for $48-72 \mathrm{~h}$. The reaction mixture was quenched with $\mathrm{NaHCO}_{3}(5 \%)$ and extracted with EtOAc $(3 \times 70 \mathrm{~mL})$. The combined organic extracts were washed successively with $\mathrm{NaHCO}_{3}(5 \%, 100 \mathrm{~mL})$, water $(100 \mathrm{~mL})$, brine $(100 \mathrm{~mL})$, and dried $\left(\mathrm{MgSO}_{4}\right)$. The solvent was evaporated in vacuo and the residue was subjected to column chromatography on silica gel.

\subsubsection{7- $N-\left(N^{\prime}-{ }^{t}\right.$ Butoxycarbonyl- $\beta$-alanyl)amino-2- ${ }^{t}$ butyl-3H-phenoxazin-3-one $20 \mathrm{a}$}

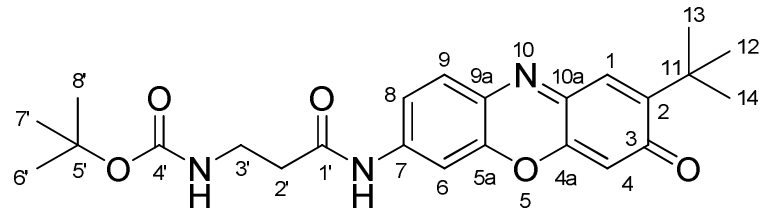

Prepared (method $\boldsymbol{A}$ ) from 7-amino-2-butyl-3H-phenoxazin-3-one 17a $(0.52 \mathrm{~g}, 1.92 \mathrm{mmol})$. Elution with a gradient mixture of petroleum ether $\left(60-80^{\circ} \mathrm{C}\right)$ : EtOAc (50:50 to 30:70) yielded 7- $N$-( $N^{\prime}$-'butoxycarbonyl- $\beta$-alanyl)amino-2-'butyl-3H-phenoxazin-3-one 20 a an orange solid (0.38g, 0.87mmol, 45.3\%); mp 194.5-197. $5^{\circ} \mathrm{C}$; (found: $\mathrm{C}, 65.3 ; \mathrm{H}, 6.7 ; \mathrm{N}, 9.4 \%$. $\mathrm{C}_{24} \mathrm{H}_{29} \mathrm{~N}_{3} \mathrm{O}_{5}$ requires C, 65.6; H, 6.65; N, 9.6\%); m/Z $438.1(M-H)^{-} ; 440.2(M H)^{+} ; v_{\max } / \mathrm{cm}^{-1}$ 3373 and $3311(\mathrm{~N}-\mathrm{H}), 3001-2883(\mathrm{C}-\mathrm{H}), 1712,1685$ and $1644(\mathrm{C}=\mathrm{O}), 1165(\mathrm{C}-\mathrm{O}) ; \delta_{\mathrm{H}}(300$ MHz, d 6 -DMSO) $1.32\left(9 \mathrm{H}, \mathrm{s}, 12 / 13 / 14-\mathrm{C}\left(\mathrm{CH}_{3}\right)_{3}\right), 1.39\left(9 \mathrm{H}, \mathrm{s}, 6^{\prime} / 7^{\prime} / 8^{\prime}-\mathrm{C}\left(\mathrm{CH}_{3}\right)_{3},\right), 2.56(2 \mathrm{H}, \mathrm{t}$, $\left.J=6.9 \mathrm{~Hz}, 2^{\prime}-\mathrm{H}\right), 3.26\left(2 \mathrm{H}, \mathrm{q}, J=6.9 \mathrm{~Hz}, 3^{\prime}-\mathrm{H}\right), 6.16(1 \mathrm{H}, \mathrm{s}, 4-\mathrm{H}), 6.88\left(1 \mathrm{H}\right.$, br. s, N $H^{t}$ Boc $)$, $7.20(1 \mathrm{H}, \mathrm{s}, 1-\mathrm{H}), 7.47(1 \mathrm{H}, \mathrm{dd}, J=8.7,2.1 \mathrm{~Hz}, 8-\mathrm{H}), 7.69(1 \mathrm{H}, \mathrm{d}, J=8.7 \mathrm{~Hz}, 9-\mathrm{H}), 7.87(1 \mathrm{H}$, $\mathrm{d}, J=2.1 \mathrm{~Hz}, 6-\mathrm{H}), 10.485(1 \mathrm{H}, \mathrm{s}, \mathrm{N} H-\mathrm{Ar}) ; \delta_{\mathrm{C}}\left(75 \mathrm{MHz}, \mathrm{d}_{6}-\mathrm{DMSO}\right) 28.7\left(\mathrm{CH}_{3}, 12 / 13 / 14-\right.$ $\left.\mathrm{C}\left(\mathrm{CH}_{3}\right)_{3}\right), 29.4\left(\mathrm{CH}_{3}, 6^{\prime} / 7^{\prime} / 8^{\prime}-\mathrm{C}\left(\mathrm{CH}_{3}\right)_{3}\right), 35.6$ (quat., 11-C( $\left.\left(\mathrm{CH}_{3}\right)_{3}\right), 36.8\left(\mathrm{CH}_{2}, 3^{\prime}-\mathrm{C}\right), 37.5\left(\mathrm{CH}_{2}\right.$, 2'-C), 78.2 (quat., 5'- $\left.\mathrm{C}\left(\mathrm{CH}_{3}\right)_{3}\right), 105.35(\mathrm{CH}, 6-\mathrm{C}), 107.5(\mathrm{CH}, 4-\mathrm{C}), 116.7(\mathrm{CH}, 8-\mathrm{C}), 129.7$ 
(quat., 7-C), 129.8 (CH, 1-C), 130.7 (CH, 9-C), 143.5 (quat., 9a-C), 144.4 (quat., 5a-C), 146.9 (quat., 4a-C), 148.75 (quat.,10a-C), 153.1 (quat., 2-C), 156.05 (quat., 1'-C), 170.8 (quat., 4'C), 185.3 (quat., 3-C).

\subsubsection{7- $N-\left(N^{\prime}-{ }^{t}\right.$ Butoxycarbonyl- $\beta$-alanyl)amino-2-pentyl-3H-phenoxazin-3-one $20 \mathrm{~b}$}

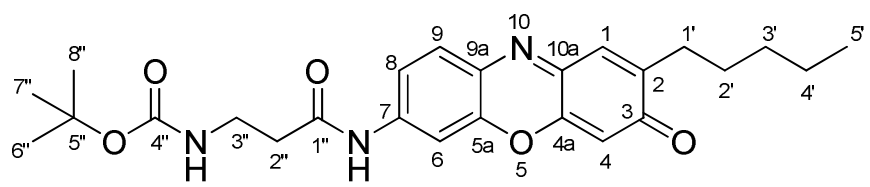

Prepared (method $\boldsymbol{A})$ from 7-amino-2-pentyl-3H-phenoxazin-3-one 17b $(0.48 \mathrm{~g}, 1.69 \mathrm{mmol})$. Elution with petroleum ether $\left(60-80^{\circ} \mathrm{C}\right)$ : EtOAc $(40: 60)$ yielded $7-N-\left(N^{\prime}-{ }^{t}\right.$ butoxycarbonyl- $\beta-$ alanyl)amino-2-pentyl-3H-phenoxazin-3-one $\mathbf{2 0 b}$ as an orange solid $(0.36 \mathrm{~g}, 0.78 \mathrm{mmol}$, 46.0\%). Recrystallization from EtOAc produced a microcrystalline orange solid with a green metallic shine; mp 205-209 ${ }^{\circ} \mathrm{C}$; (found: $\mathrm{C}, 66.35 ; \mathrm{H}, 6.9 ; \mathrm{N}, 9.25 \% . \mathrm{C}_{25} \mathrm{H}_{21} \mathrm{~N}_{3} \mathrm{O}_{5}$ requires $\mathrm{C}$, 66.2; H, 6.9; N, 9.3\%); $m / Z 453.2(M-H)^{-} ; 454.3(M H)^{+} ; v_{\max } / \mathrm{cm}^{-1} 3263$ and $3190(\mathrm{~N}-\mathrm{H}), 2951$ and $2922(\mathrm{C}-\mathrm{H}), 1710$ and $1651(\mathrm{C}=\mathrm{O})$; $\delta_{\mathrm{H}}\left(300 \mathrm{MHz}, \mathrm{d}_{6}-\mathrm{DMSO}\right) 0.79\left(3 \mathrm{H}, \mathrm{t}, J=6.9 \mathrm{~Hz}, 5^{\prime}-\right.$ H), 1.20-1.245 (4H, m, 3'-H and 4'-H), 1.30 (9H, s, C(CH3) $), 1.39-1.46\left(2 \mathrm{H}, \mathrm{m}, 2^{\prime}-\mathrm{H}\right), 2.39$ $\left(2 \mathrm{H}, \mathrm{t}, J=7.5 \mathrm{~Hz}, 1^{\prime}-\mathrm{H}\right), 2.47\left(2 \mathrm{H}, \mathrm{t}, J=7.2 \mathrm{~Hz}, 2^{\prime \prime}-\mathrm{H}\right), 3.17(2 \mathrm{H}, \mathrm{q}, J=7.2 \mathrm{~Hz}, 3 "-\mathrm{H}), 6.20$ $(1 \mathrm{H}, \mathrm{s}, 4-\mathrm{H}), 6.80\left(1 \mathrm{H}\right.$, br. s, NH $\left.H^{t} \mathrm{Boc}\right), 7.22(1 \mathrm{H}, \mathrm{s}, 1-\mathrm{H}), 7.40(1 \mathrm{H}, \mathrm{dd}, J=8.7,2.1 \mathrm{~Hz}, 7-\mathrm{H})$, $7.66(1 \mathrm{H}, \mathrm{d}, J=8.7 \mathrm{~Hz}, 9-\mathrm{H}), 7.82(1 \mathrm{H}, \mathrm{d}, J=2.1 \mathrm{~Hz}, 6-\mathrm{H}), 10.41(1 \mathrm{H}, \mathrm{s}, \mathrm{N} H-\mathrm{Ar}) ; \delta_{\mathrm{C}}(75$ MHz, d $6_{6}$-DMSO $14.3\left(\mathrm{CH}_{3}, 5^{\prime}-\mathrm{C}\right), 22.4\left(\mathrm{CH}_{2}, 3^{\prime}-\mathrm{C}\right.$ or 4'-C $), 28.1\left(\mathrm{CH}_{2}, 2^{\prime}-\mathrm{C}\right), 28.7\left(\mathrm{CH}_{2}, 1^{\prime}-\right.$ C), $29.4\left(\mathrm{CH}_{3}, 6 " / 7 " / 8 "-\mathrm{C}\left(\mathrm{CH}_{3}\right)_{3}\right), 31.5\left(\mathrm{CH}_{2}, 3 '-\mathrm{C}\right.$ or 4'-C), $36.8\left(\mathrm{CH}_{2}, 3 "-\mathrm{C}\right), 37.5\left(\mathrm{CH}_{2}, 2^{\prime \prime}-\right.$ C), 78.2 (quat., 5"- $\left.\left(\mathrm{CH}_{3}\right)_{3}\right), 105.3(\mathrm{CH}, 6-\mathrm{C}), 106.05(\mathrm{CH}, 4-\mathrm{C}), 116.8(\mathrm{CH}, 8-\mathrm{C}), 129.6$ (quat.,7-C), 130.7 (CH, 9-C), 130.9 (CH, 1-C), 143.4 (quat., 9a-C), 144.5 (quat., 5a-C), 146.7 (quat., 10a-C and 2-C), 149.5 (quat., 4a-C), 156.0 (quat., 4"-C), 170.8 (quat., 1"-C), 185.1 (quat., 3-C).

\subsubsection{8- $N$-( $N^{\prime}-{ }^{t}$ Butoxycarbonyl- $\beta$-alanyl)amino-3H-phenoxazin-3-one 21 a}

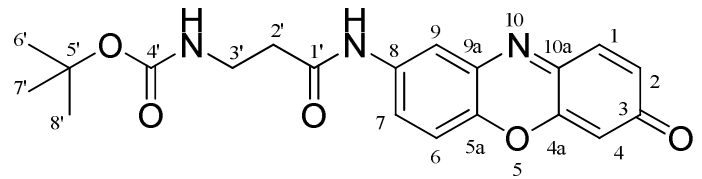

Prepared (method B) from 8-amino-3H-phenoxazin-3-one 2a $(0.37 \mathrm{~g}, 1.75 \mathrm{mmol})$. Elution with a gradient mixture of petroleum ether $\left(60-80^{\circ} \mathrm{C}\right)$ : EtOAc $(20: 80$ to $0: 100)$ yielded $8-N-\left(N^{\prime}-\right.$ ${ }^{t}$ butoxycarbonyl- $\beta$-alanyl)amino-3H-phenoxazin-3-one 21a as a dark orange solid $(0.36 \mathrm{~g}$, 
0.95mmol, 54.3\%); mp 213-215 ${ }^{\circ}$; (found: $\mathrm{C}, 62.3 ; \mathrm{H}, 5.6 ; \mathrm{N}, 10.7 \% . \mathrm{C}_{20} \mathrm{H}_{21} \mathrm{~N}_{3} \mathrm{O}_{5}$ requires C, 62.65; H, 5.5; N, 11.0\%); $m / z 384.1(M H)^{+}, 406.1(M N a)^{+} ; v_{\max } / \mathrm{cm}^{-1} 3385$ and $3304(\mathrm{~N}-\mathrm{H})$, 1683 and $1644(\mathrm{C}=\mathrm{O}), 1172$ and $1136(\mathrm{C}-\mathrm{O}) ; \delta_{\mathrm{H}}\left(300 \mathrm{MHz}, \mathrm{d}_{6}-\mathrm{DMSO}\right) 1.38\left(9 \mathrm{H}, \mathrm{s}, \mathrm{C}\left(\mathrm{CH}_{3}\right)_{3}\right)$, $2.51\left(2 \mathrm{H}, \mathrm{t}, J=7.8 \mathrm{~Hz}, 2^{\prime}-\mathrm{H}\right), 3.25\left(2 \mathrm{H}, \mathrm{q}, J=6.9 \mathrm{~Hz}, 3^{\prime}-\mathrm{H}\right), 6.24(1 \mathrm{H}, \mathrm{d}, J=2.1 \mathrm{~Hz}, 4-\mathrm{H}), 6.81$ $(1 \mathrm{H}, \mathrm{dd}, J=9.9,2.1 \mathrm{~Hz}, 2-\mathrm{H}), 6.86\left(1 \mathrm{H}\right.$, br. s, $\left.\mathrm{N} H^{t} \mathrm{Boc}\right), 7.42(1 \mathrm{H}, \mathrm{d}, J=8.7 \mathrm{~Hz}, 6-\mathrm{H}), 7.52$ $(1 \mathrm{H}, \mathrm{d}, J=9.9 \mathrm{~Hz}, 1-\mathrm{H}), 7.73(1 \mathrm{H}, \mathrm{dd}, J=9.0,2.7 \mathrm{~Hz}, 7-\mathrm{H}), 8.14(1 \mathrm{H}, \mathrm{d}, J=2.7 \mathrm{~Hz}, 9-\mathrm{H})$, $10.20(1 \mathrm{H}, \mathrm{s}, \mathrm{NH}-\mathrm{Ar}) ; \delta_{\mathrm{C}}\left(75 \mathrm{MHz}, \mathrm{d}_{6}\right.$-DMSO) $28.75\left(\mathrm{CH}_{3}, 6^{\prime} / 7^{\prime} / 8^{\prime}-\mathrm{C}\left(\mathrm{CH}_{3}\right)_{3}\right), 37.0\left(\mathrm{CH}_{2}, 3^{\prime}-\mathrm{C}\right)$, $37.35\left(\mathrm{CH}_{2}, 2\right.$ '-C), 78.2 (quat., 5'- $\left.\mathrm{C}\left(\mathrm{CH}_{3}\right)_{3}\right), 106.1(\mathrm{CH}, 4-\mathrm{C}), 116.6(\mathrm{CH}, 6-\mathrm{C}), 119.3(\mathrm{CH}, 9-$ C), 124.7 (CH, 7-C), 133.3 (quat., 9a-C), 135.3 (2 × CH, 1-C and 2-C), 137.1 (quat., 8-C), 139.65 (quat., 5a-C), 149.2 (quat., 10a-C), 150.1 (quat., 4a-C), 156.1 (quat., 4'-C), 170.2 (quat., 1'-C), 185.9 (quat., 3-C).

\subsubsection{8- $N-\left(N^{\prime}-{ }^{t}\right.$ Butoxycarbonyl- $\beta$-alanyl)amino-1,2,4-trimethyl-3H-phenoxazin-3-one} $21 b$

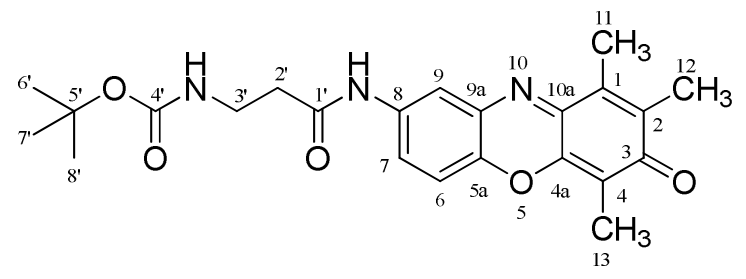

Prepared (method B) from 8-amino-1,2,4-trimethyl-3H-phenoxazin-3-one 2b $(0.67 \mathrm{~g}$, $2.65 \mathrm{mmol})$. Elution with petroleum ether $\left(60-80^{\circ} \mathrm{C}\right)$ : EtOAc $(30: 70)$ yielded $8-N-\left(N^{\prime}-\right.$ ${ }^{t}$ butoxycarbonyl- $\beta$-alanyl)amino-1,2,4-trimethyl-3H-phenoxazin-3-one $\quad \mathbf{2 1 b} \quad(0.38 \mathrm{~g}$, $0.98 \mathrm{mmol}, 37.0 \%$ ) as a dark orange solid. Recrystallization from EtOAc produced a microcrystalline orange solid; $\mathrm{mp} 195-198^{\circ} \mathrm{C}$; (found: $\mathrm{C}, 64.75 ; \mathrm{H}, 6.65 ; \mathrm{N}, 9.7 \%$. $\mathrm{C}_{23} \mathrm{H}_{27} \mathrm{~N}_{3} \mathrm{O}_{5}$ requires C, 64.9; H, 6.4; N, 9.9\%); $\mathrm{m} / \mathrm{Z} 426.2(M H)^{+}, 448.2(M N a)^{+} ; v_{\max } / \mathrm{cm}^{-1}$ $3333(\mathrm{~N}-\mathrm{H}), 1713,1690$ and $1650(\mathrm{C}=\mathrm{O}), 1167(\mathrm{C}-\mathrm{O})$; $\delta_{\mathrm{H}}\left(300 \mathrm{MHz}, \mathrm{d}_{6}-\mathrm{DMSO}\right) 1.42(9 \mathrm{H}, \mathrm{s}$, $\left.\mathrm{C}\left(\mathrm{CH}_{3}\right)_{3}\right), 1.98(3 \mathrm{H}, \mathrm{s}, 13-\mathrm{H}), 2.07(3 \mathrm{H}, \mathrm{s}, 12-\mathrm{H}), 2.33(3 \mathrm{H}, \mathrm{s}, 11-\mathrm{H}), 2.545(2 \mathrm{H}, \mathrm{t}, J=6.9 \mathrm{~Hz}$, 2'-H), 3.32 (2H, q, $\left.J=6.6 \mathrm{~Hz}, 3^{\prime}-\mathrm{H}\right), 6.49$ (1H, br. s, N $H^{t}$ Boc), $7.32(1 \mathrm{H}, \mathrm{d}, J=8.7 \mathrm{~Hz}, 6-\mathrm{H})$, $7.64(1 \mathrm{H}, \mathrm{dd}, J=8.7,2.1 \mathrm{~Hz}, 7-\mathrm{H}), 8.09(1 \mathrm{H}, \mathrm{d}, J=1.8 \mathrm{~Hz}, 9-\mathrm{H}), 9.90(1 \mathrm{H}, \mathrm{s}, \mathrm{Ar}-\mathrm{N} H \mathrm{CO}) ; \delta_{\mathrm{C}}$ (75 MHz, d 6 -DMSO) $7.9\left(\mathrm{CH}_{3}, 13-\mathrm{C}\right), 12.8\left(\mathrm{CH}_{3}, 11-\mathrm{C}\right), 13.2\left(\mathrm{CH}_{3}, 12-\mathrm{C}\right), 28.8\left(\mathrm{CH}_{3}\right.$, 6'/7'/8'-C(CH$\left.)_{3}\right), 37.3\left(\mathrm{CH}_{2}, 3^{\prime}-\mathrm{C}\right), 37.5\left(\mathrm{CH}_{2}, 2^{\prime}-\mathrm{C}\right), 78.3$ (quat., 5'-C( $\left.\left.\mathrm{CH}_{3}\right)_{3}\right), 113.0$ (quat., 4 C), $116.0(\mathrm{CH}, 6-\mathrm{C}), 119.8(\mathrm{CH}, 9-\mathrm{C}), 123.7(\mathrm{CH}, 7-\mathrm{C}), 132.55$ (quat., 9a-C), 136.55 (quat., 1-C or 2-C), 136.9 (quat., 8-C), 138.9 (quat., 1-C or 2-C), 140.1 (quat., 5a-C), 145.6 (quat., 4a-C), 149.1 (quat., 10a-C), 156.0 (quat., 4'-C), 170.1 (quat., 1'-C), 184.4 (quat., 3-C). 


\subsubsection{8- $N$-( $N^{\prime}-{ }^{t}$ Butoxycarbonyl- $\beta$-alanyl)amino-1,2-dimethyl-3H-phenoxazin-3-one 21c}

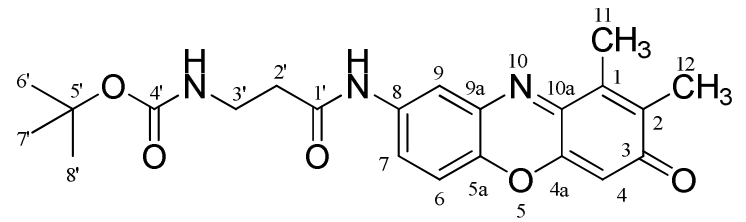

Prepared (method B) from 8-amino-1,2-dimethyl-3H-phenoxazin-3-one 2c $(0.27 \mathrm{~g}$, $1.14 \mathrm{mmol})$. Elution with petroleum ether $\left(60-80^{\circ} \mathrm{C}\right)$ : EtOAc : $(30: 70)$ yielded $8-N-\left(N^{\prime}-\right.$ tbutoxycarbonyl- $\beta$-alanyl)amino-1,2-dimethyl-3H-phenoxazin-3-one 21c as a bright orange solid $(0.46 \mathrm{~g}, 1.12 \mathrm{mmol}, 98.3 \%)$. Recrystallization from EtOAc produced a microcrystalline orange solid; mp $216-217^{\circ} \mathrm{C}$; (found: $\mathrm{C}, 64.2 ; \mathrm{H}, 6.2 ; \mathrm{N}, 10.2 \% . \mathrm{C}_{22} \mathrm{H}_{25} \mathrm{~N}_{3} \mathrm{O}_{5}$ requires $\mathrm{C}$, 64.2; $\mathrm{H}, 6.1 ; \mathrm{N}, 10.2 \%) ; \mathrm{m} / \mathrm{Z} 412.1(\mathrm{MH})^{+}, 434.1(\mathrm{MNa})^{+} ; v_{\max } / \mathrm{cm}^{-1} 3337(\mathrm{~N}-\mathrm{H}), 2973$ and $2927(\mathrm{C}-$ $\mathrm{H}), 1700,1679$ and $1647(\mathrm{C}=\mathrm{O}), 1166(\mathrm{C}-\mathrm{O})$; $\delta_{\mathrm{H}}$ (300 MHz, d $\left.\mathrm{d}_{6} \mathrm{DMSO}\right) 1.31$ (9H, s, $\left.\mathrm{C}\left(\mathrm{CH}_{3}\right)_{3}\right), 1.95\left(3 \mathrm{H}, \mathrm{s}, \mathrm{CH}_{3}, 12-\mathrm{H}\right), 2.24\left(3 \mathrm{H}, \mathrm{s}, \mathrm{CH}_{3}, 11-\mathrm{H}\right), 2.425\left(2 \mathrm{H}, \mathrm{t}, J=7.2 \mathrm{~Hz}, \mathrm{CH}_{2}, 2^{\prime}-\right.$ H), $3.175\left(2 \mathrm{H}, \mathrm{q}, J=7.2 \mathrm{~Hz}, 3^{\prime}-\mathrm{H}\right), 6.06(1 \mathrm{H}, \mathrm{s}, 4-\mathrm{H}), 6.78\left(1 \mathrm{H}\right.$, br. s, NH $\left.H^{t} \mathrm{Boc}\right), 7.28(1 \mathrm{H}, \mathrm{d}, J$ $=8.7 \mathrm{~Hz}, 6-\mathrm{H}), 7.53(1 \mathrm{H}, \mathrm{dd}, J=9.0,2.4 \mathrm{~Hz}, 7-\mathrm{H}), 8.08(1 \mathrm{H}, \mathrm{d}, J=2.4 \mathrm{~Hz}, 9-\mathrm{H}), 10.06(1 \mathrm{H}, \mathrm{s}$, $\mathrm{N} H-\mathrm{Ar}) ; \delta_{\mathrm{C}}\left(75 \mathrm{MHz}, \mathrm{d}_{6}-\mathrm{DMSO}\right) 13.0\left(\mathrm{CH}_{3}, 11-\mathrm{C}\right.$ or $\left.12-\mathrm{C}\right), 13.15\left(\mathrm{CH}_{3}, 11-\mathrm{C}\right.$ or 12-C), 28.7 $\left(\mathrm{CH}_{3}, 66^{\prime} / 7^{\prime} / 8^{\prime}-\mathrm{C}\left(\mathrm{CH}_{3}\right)_{3}\right), 37.0\left(\mathrm{CH}_{2}, 3^{\prime}-\mathrm{C}\right), 37.3\left(\mathrm{CH}_{2}, 2^{\prime}-\mathrm{C}\right), 78.15$ (quat., 5'-C( $\left.\left.\mathrm{CH}_{3}\right)_{3}\right), 104.9$ (CH, 4-C), $116.2(\mathrm{CH}, 6-\mathrm{C}), 119.3(\mathrm{CH}, 9-\mathrm{C}), 123.8$ (CH, 7-C), 132.7 (quat., 9a-C), 136.8 (quat., 8-C), 137.8 (quat., 1-C), 139.4 (quat., 2-C or 5a-C), 139.5 (quat., 2-C or 5a-C), 149.0 (quat., 10a-C), 149.4 (quat., 4a-C), 156.05 (quat., 4'-C), 170.1 (quat., 1'-C), 184.8 (quat., 3-C).

\subsubsection{8- $N$-( $N^{\prime}-{ }^{t}$ Butoxycarbonyl- $\beta$-alanyl)amino-2- ${ }^{t}$ butyl-3H-phenoxazin-3-one $21 \mathrm{~d}$}

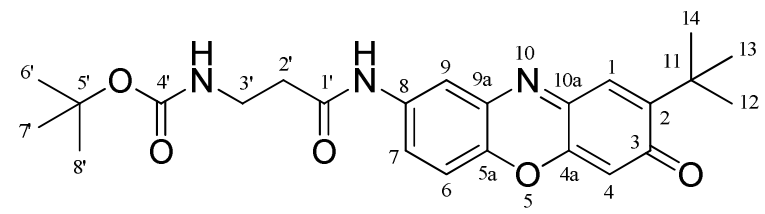

Prepared (method B) from 8-amino-2-'butyl-3H-phenoxazin-3-one 2d (0.53g, 1.96mmol). Elution with petroleum ether $\left(60-80^{\circ} \mathrm{C}\right)$ : EtOAc (30:70) yielded 8- $N$ - $\left(N^{\prime}-{ }^{t}\right.$ butoxycarbonyl- $\beta-$ alanyl)amino-2- ${ }^{t}$ butyl-3 $H$-phenoxazin-3-one $21 \mathrm{~d}(0.53 \mathrm{~g}, 1.21 \mathrm{mmol}, 61.7 \%)$ as a dark orange solid; mp 191.5-193.5 ${ }^{\circ} \mathrm{C}$; (found: $\mathrm{C}, 65.5 ; \mathrm{H}, 6.8 ; \mathrm{N}, 9.5 \% . \mathrm{C}_{24} \mathrm{H}_{29} \mathrm{~N}_{3} \mathrm{O}_{5}$ requires $\mathrm{C}, 65.6 ; \mathrm{H}$, 6.65; N, 9.6\%); m/Z 438.1 (M-H); $v_{\max } / \mathrm{cm}^{-1} 3340$ and $3304(\mathrm{~N}-\mathrm{H}), 2962(\mathrm{C}-\mathrm{H}), 1682$ and $1648(\mathrm{C}=\mathrm{O}), 1167(\mathrm{C}-\mathrm{O}) ; \delta_{\mathrm{H}}\left(300 \mathrm{MHz}, \mathrm{d}_{6}\right.$-DMSO) $1.375\left(9 \mathrm{H}, \mathrm{s}, 12 / 13 / 14-\mathrm{C}\left(\mathrm{CH}_{3}\right)_{3}\right), 1.44$ $\left(9 \mathrm{H}, \mathrm{s}, 6^{\prime} / 7^{\prime} / 8^{\prime}-\mathrm{C}\left(\mathrm{CH}_{3}\right)_{3}\right), 2.57\left(2 \mathrm{H}, \mathrm{t}, J=7.2 \mathrm{~Hz}, 2^{\prime}-\mathrm{H}\right), 3.31\left(2 \mathrm{H}, \mathrm{q}, J=7.2 \mathrm{~Hz}, 3^{\prime}-\mathrm{H}\right), 6.21(1 \mathrm{H}$, s, 4-H), 6.90 (1H, br. s, NH $H^{t}$ Boc), 7.29 (1H, s, 1-H), 7.78 (1H, dd, $\left.J=9.0,2.4 \mathrm{~Hz}, 7-\mathrm{H}\right), 8.17$ 
$(1 \mathrm{H}, \mathrm{d}, J=2.4 \mathrm{~Hz}, 9-\mathrm{H}), 10.24(1 \mathrm{H}, \mathrm{s}, \mathrm{N} H-\mathrm{Ar}) ; \delta_{\mathrm{C}}\left(75 \mathrm{MHz}, \mathrm{d}_{6}\right.$-DMSO) $28.7\left(\mathrm{CH}_{3}, 6^{\prime} / 7^{\prime} / 8^{\prime}-\right.$ $\left.\mathrm{C}\left(\mathrm{CH}_{3}\right)_{3}\right), 29.4\left(\mathrm{CH}_{3}, 12 / 13 / 14-\mathrm{C}\left(\mathrm{CH}_{3}\right)_{3}\right), 35.7$ (quat., 11- $\left.\mathrm{C}\left(\mathrm{CH}_{3}\right)_{3}\right), 37.0\left(\mathrm{CH}_{2}, 3\right.$ '-C), 37.3 $\left(\mathrm{CH}_{2}, 2^{\prime}-\mathrm{C}\right), 78.1$ (quat., 5'-C( $\left.\left.\mathrm{CH}_{3}\right)_{3}\right), 107.4(\mathrm{CH}, 4-\mathrm{C}), 116.5(\mathrm{CH}, 6-\mathrm{C}), 119.1(\mathrm{CH}, 9-\mathrm{C})$, 124.1 (CH, 7-C), 129.8 (CH, 1-C), 133.5 (quat., 9a-C), 137.0 (quat., 8-C), 139.4 (quat., 5a-C), 148.0 (quat., 4a-C or 10a-C), 149.6 (quat., 4a-C or 10a-C), 154.05 (quat., 2-C), 156.05 (quat., 4'-C), 170.1 (quat., 1'-C), 185.5 (quat., 3-C).

\subsubsection{8- $N$-( $N^{\prime}-{ }^{t}$ Butoxycarbonyl- $\beta$-alanyl)amino-2-pentyl-3H-phenoxazin-3-one $21 \mathrm{e}$}

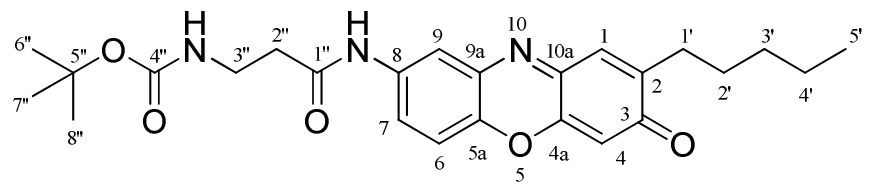

Prepared (method B) from 8-amino-2-pentyl-3H-phenoxazin-3-one 2e (0.50g, 1.78mmol). Elution with a gradient mixture of petroleum ether $\left(60-80^{\circ} \mathrm{C}\right)$ : EtOAc (35:65 to 20:80) yielded 8-N-( $N^{\prime}-$ tbutoxycarbonyl- $\beta$-alanyl)amino-2-pentyl-3H-phenoxazin-3-one $21 \mathrm{e}$ as an orange solid $(0.73 \mathrm{~g}, 1.60 \mathrm{mmol}, 90.1 \%)$; mp 176-179 ${ }^{\circ} \mathrm{C}$; (found: $\mathrm{C}, 66.2 ; \mathrm{H}, 6.8 ; \mathrm{N}, 9.1 \%$. $\mathrm{C}_{25} \mathrm{H}_{31} \mathrm{~N}_{3} \mathrm{O}_{5}$ requires C, 66.2; H, 6.9; N, 9.3\%); m/z $454.3(M H)^{+}, 476.3(M N a)^{+} ; v_{\max } / \mathrm{cm}^{-1}$ $3322(\mathrm{~N}-\mathrm{H}), 2954$ and $2930(\mathrm{C}-\mathrm{H}), 1684,1658$ and $1648(\mathrm{C}=\mathrm{O}), 1171(\mathrm{C}-\mathrm{O}) ; \delta_{\mathrm{H}}(300 \mathrm{MHz}$, $\mathrm{d}_{6}$-DMSO $0.78\left(3 \mathrm{H}, \mathrm{t}, J=6.6 \mathrm{~Hz}, 5^{\prime}-\mathrm{H}\right), 1.21-1.23\left(4 \mathrm{H}, \mathrm{m}, 3^{\prime}-\mathrm{H}\right.$ and $\left.4 '-\mathrm{H}\right), 1.30(9 \mathrm{H}, \mathrm{s}$, $\left.\mathrm{C}\left(\mathrm{CH}_{3}\right)_{3}\right), 1.38-1.40\left(2 \mathrm{H}, \mathrm{m}, 2^{\prime}-\mathrm{H}\right), 2.34\left(2 \mathrm{H}, \mathrm{t}, J=7.2 \mathrm{~Hz}, 1^{\prime}-\mathrm{H}\right), 2.42\left(2 \mathrm{H}, \mathrm{t}, J=6.6 \mathrm{~Hz}, 2^{\prime \prime}-\mathrm{H}\right)$, $3.19(2 \mathrm{H}, \mathrm{q}, J=6.6 \mathrm{~Hz}, 3 "-\mathrm{H}), 6.095(1 \mathrm{H}, \mathrm{s}, 4-\mathrm{H}), 6.77$ (1H, br. s, NH $\left.H^{\mathrm{t} B o c}\right), 7.15(1 \mathrm{H}, \mathrm{s}, 1-\mathrm{H})$, $7.28(1 \mathrm{H}, \mathrm{d}, J=8.7 \mathrm{~Hz}, 6-\mathrm{H}), 7.60(1 \mathrm{H}, \mathrm{dd}, J=8.7,2.4 \mathrm{~Hz}, 7-\mathrm{H}), 8.00(1 \mathrm{H}, \mathrm{d}, J=2.1 \mathrm{~Hz}, 9-\mathrm{H})$, $10.08(1 \mathrm{H}, \mathrm{s}, \mathrm{N} H-\mathrm{Ar}) ; \delta_{\mathrm{C}}\left(75 \mathrm{MHz}, \mathrm{d}_{6}-\mathrm{DMSO}\right) 14.3\left(\mathrm{CH}_{3}, 5^{\prime}-\mathrm{C}\right), 22.4\left(\mathrm{CH}_{2}, 3^{\prime}-\mathrm{C}\right.$ or 4'-C), $28.0\left(\mathrm{CH}_{2}, 2^{\prime}-\mathrm{C}\right), 28.7\left(\mathrm{CH}_{3}, 6 " / 7 " / 8 "-\mathrm{C}\left(\mathrm{CH}_{3}\right)_{3}\right), 29.4\left(\mathrm{CH}_{2}, 1^{\prime}-\mathrm{C}\right), 31.5\left(\mathrm{CH}_{2}, 3 '-\mathrm{C}\right.$ or 4'-C), $36.95\left(\mathrm{CH}_{2}, 3\right.$ "-C), $37.3\left(\mathrm{CH}_{2}, 2\right.$ "-C), 78.1 (quat., 5"-C( $\left.\left(\mathrm{CH}_{3}\right)_{3}\right), 105.8(\mathrm{CH}, 4-\mathrm{C}), 116.5(\mathrm{CH}$, 6-C), 119.0 (CH, 9-C), 124.0 (CH, 7-C), 130.7 (CH, 1-C), 133.3 (quat., 9a-C), 137.0 (quat., 8C), 139.3 (quat., 5a-C), 147.6 (quat., 2-C), 149.2 (quat., 4a-C or 10a-C), 149.3 (quat., $4 \mathrm{a}-\mathrm{C}$ or 10a-C), 156.0 (quat., 4"-C), 170.05 (quat., 1"-C), 185.1 (quat., 3-C).

\subsubsection{8- $N-\left(N^{\prime}-{ }^{t}\right.$ Butoxycarbonyl- $\beta$-alanyl)amino-1-phenyl-3H-phenoxazin-3-one $21 f$}

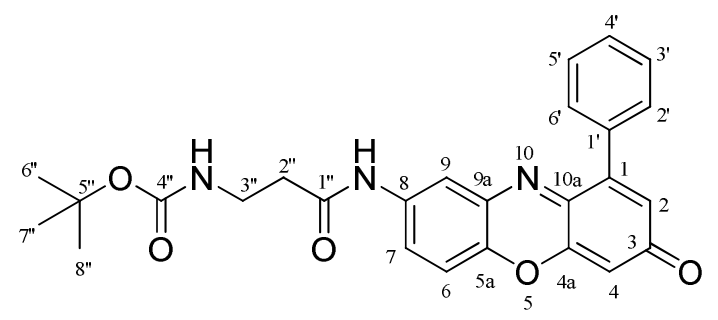


Prepared (method B) from 8-amino-1-phenyl-3H-phenoxazin-3-one 2f $(0.12 \mathrm{~g}, 0.39 \mathrm{mmol})$. Elution used a gradient mixture of petroleum ether $\left(60-80^{\circ} \mathrm{C}\right)$ : EtOAc (50:50 to 40:60) yielding 8- $N$-( $\left(N^{\prime}\right.$-'butoxycarbonyl- $\beta$-alanyl)amino-1-phenyl-3H-phenoxazin-3-one $21 \mathbf{f}$ as an orange-red solid $(0.12 \mathrm{~g}, 0.27 \mathrm{mmol}, 69.6 \%) ; \mathrm{mp} 241-243^{\circ} \mathrm{C}$; (found: $\mathrm{C}, 67.7 ; \mathrm{H}, 5.6 ; \mathrm{N}, 8.9 \%$. $\mathrm{C}_{26} \mathrm{H}_{25} \mathrm{~N}_{3} \mathrm{O}_{5}$ requires $\left.\mathrm{C}, 68.0 ; \mathrm{H}, 5.5 ; \mathrm{N}, 9.1 \%\right) ; \mathrm{m} / \mathrm{Z} 460.2(M H)^{+} ; v_{\max } / \mathrm{cm}^{-1} 3422$ and 3263 $(\mathrm{N}-\mathrm{H}), 1679$ and $1639(\mathrm{C}=\mathrm{O}), 1167(\mathrm{C}-\mathrm{O}) ; \delta_{\mathrm{H}}\left(300 \mathrm{MHz}, \mathrm{d}_{6}-\mathrm{DMSO}\right) 1.385\left(9 \mathrm{H}, \mathrm{s}, \mathrm{C}\left(\mathrm{CH}_{3}\right)_{3}\right)$, $2.50(2 \mathrm{H}, \mathrm{t}, J=7.2 \mathrm{~Hz}, 2 "-\mathrm{H}), 3.24(2 \mathrm{H}, \mathrm{q}, J=6.9 \mathrm{~Hz}, 3 "-\mathrm{H}), 6.32(1 \mathrm{H}, \mathrm{d}, J=2.1 \mathrm{~Hz}, 4-\mathrm{H})$, $6.80(1 \mathrm{H}, \mathrm{d}, J=2.1 \mathrm{~Hz}, 2-\mathrm{H}), 6.86\left(1 \mathrm{H}\right.$, br. s, $\left.\mathrm{N} H^{t} \mathrm{Boc}\right), 7.45(1 \mathrm{H}, \mathrm{d}, J=9.0 \mathrm{~Hz}, 6-\mathrm{H}), 7.49-7.51$ $\left(3 \mathrm{H}, \mathrm{m}, 3{ }^{\prime}-\mathrm{H}, 4^{\prime}-\mathrm{H}\right.$ and 5'-H), 7.59-7.63 (2H, m, 2'-H and 6'-H), $7.68(1 \mathrm{H}, \mathrm{dd}, J=9.0,2.4 \mathrm{~Hz}$, 7-H), $8.12(1 \mathrm{H}, \mathrm{d}, J=2.4 \mathrm{~Hz}, 9-\mathrm{H}), 10.17(1 \mathrm{H}, \mathrm{s}, \mathrm{NH}-\mathrm{Ar}) ; \delta_{\mathrm{C}}\left(75 \mathrm{MHz}, \mathrm{d}_{6}-\mathrm{DMSO}\right) 28.7\left(\mathrm{CH}_{3}\right.$, 6"/7"/8"-C $\left.\left(\mathrm{CH}_{3}\right)_{3}\right), 37.0\left(\mathrm{CH}_{2}, 3 "-\mathrm{C}\right), 37.3\left(\mathrm{CH}_{2}, 2\right.$ "-C), 78.1 (quat., 5"-C( $\left.\left(\mathrm{CH}_{3}\right)_{3}\right), 106.0(\mathrm{CH}$, 4-C), 116.5 (CH, 6-C), 119.6 (CH, 9-C), 124.8 (CH, 7-C), 128.4 (CH, 3'-C and 5'-C), 129.3 $(\mathrm{CH}, 4$ '-C), 130.55 (CH, 2'-C and 6'-C), 133.0 (quat., 9a-C), 133.2 (CH, 2-C), 135.2 (quat., 1C), 136.95 (quat., 8-C), 139.5 (quat., 5a-C), 145.2 (quat., 1'-C), 148.25 (quat., 10a-C), 150.4 (quat., 4a-C), 156.0 (quat., 4"-C), 170.1 (quat., 1"-C), 184.9 (quat., 3-C).

\subsection{Synthesis of 7- $N$ - and 8- $N$-( $\beta$-alanyl)amino-3H-phenoxazin-3-one TFA salts 22a-b and 23a-f}

\subsubsection{General procedure}

To the appropriately substituted $7-N$ - or $8-N-\left(N^{\prime}\right.$ - butoxycarbonyl- $\beta$-alanyl)amino-alkyl-3Hphenoxazin-3-one 20a-b or 21a-f in a round bottomed flask, an excess of neat TFA was added and the resulting solution was swirled for one minute. The TFA was evaporated in vacuo, then sequential portions of $\mathrm{MeOH}$ were added to the residue and evaporated. The residue was redissolved in minimal $\mathrm{MeOH}$ and diluted with $\mathrm{Et}_{2} \mathrm{O}$. After overnight standing, the microcrystalline solid that formed was collected and washed with $\mathrm{Et}_{2} \mathrm{O}$.

\subsubsection{7- $N$-( $(\beta-A l a n y l) a m i n o-2-{ }^{t}$ butyl-3H-phenoxazin-3-one TFA salt 22a}

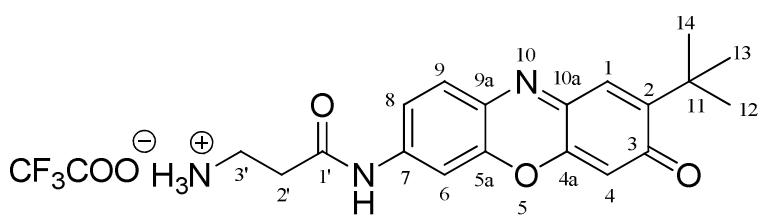

Prepared from 7- $N$ - $\left(N^{\prime}-{ }^{t}\right.$ butoxycarbonyl- $\beta$-alanyl)amino-2-'butyl-3 $H$-phenoxazin-3-one 20a $(0.11 \mathrm{~g}, 0.25 \mathrm{mmol}) .7-N$-( $\beta$-Alanyl)amino-2- ${ }^{t}$ butyl-3H-phenoxazin-3-one TFA salt 22a was isolated as dark-red solid $(0.105 \mathrm{~g}, 0.23 \mathrm{mmol}, 92.0 \%)$; mp $192-195^{\circ} \mathrm{C}$; HRMS (Found $(M)^{+}$, 
340.1657. Calc. for $\left.\mathrm{C}_{19} \mathrm{H}_{22} \mathrm{~N}_{3} \mathrm{O}_{3}:(M)^{+}, 340.1656\right) ; \mathrm{m} / \mathrm{z} 340.2\left(M^{+}\right) ; v_{\max } / \mathrm{cm}^{-1} 3180(\mathrm{~N}-\mathrm{H})$, $3028\left(\mathrm{~N}^{+}-\mathrm{H}\right), 2961(\mathrm{C}-\mathrm{H}), 1672(\mathrm{C}=\mathrm{O})$; $\delta_{\mathrm{H}}\left(300 \mathrm{MHz}, \mathrm{d}_{6}\right.$-DMSO) $1.235\left(9 \mathrm{H}, \mathrm{s}, \mathrm{C}\left(\mathrm{CH}_{3}\right)_{3}\right)$, $2.73\left(2 \mathrm{H}, \mathrm{t}, J=6.6 \mathrm{~Hz}, 2^{\prime}-\mathrm{H}\right), 3.07\left(2 \mathrm{H}, \mathrm{t}, J=6.6 \mathrm{~Hz}, 3^{\prime}-\mathrm{H}\right), 6.07(1 \mathrm{H}, \mathrm{s}, 4-\mathrm{H}), 7.12(1 \mathrm{H}, \mathrm{s}, 1-$ H), $7.42(1 \mathrm{H}, \mathrm{dd}, J=8.7,1.8 \mathrm{~Hz}, 8-\mathrm{H}), 7.62(1 \mathrm{H}, \mathrm{d}, J=8.7 \mathrm{~Hz}, 9-\mathrm{H}), 7.77(1 \mathrm{H}, \mathrm{d}, J=2.1 \mathrm{~Hz}$, 6-H), $7.84\left(3 \mathrm{H}\right.$, br. s, $\left.\mathrm{NH}_{3}{ }^{+}\right), 10.69(1 \mathrm{H}, \mathrm{s}, \mathrm{NH}-\mathrm{Ar}) ; \delta_{\mathrm{C}}\left(75 \mathrm{MHz}, \mathrm{d}_{6}-\mathrm{DMSO}\right) 29.4\left(\mathrm{CH}_{3}\right.$, 12/13/14-C $\left.\left(\mathrm{CH}_{3}\right)_{3}\right), 34.1\left(\mathrm{CH}_{2}, 2^{\prime}-\mathrm{C}\right), 35.3\left(\mathrm{CH}_{2}, 3\right.$ '-C), 35.6 (quat., 11- $\left.\mathrm{C}\left(\mathrm{CH}_{3}\right)_{3}\right), 105.7(\mathrm{CH}$, 6-C), 107.6 (CH, 4-C), 116.9 (CH, 8-C), 129.8 (CH, 1-C), 129.89 (quat., 7-C), 130.7 (CH, 9C), 143.1 (quat., 7-C), 144.4 (quat., 5a-C), 147.2 (quat., 10a-C), 148.7 (quat., 4a-C), 153.3 (quat., 2-C), 169.7 (quat., 1'-C), 185.35 (quat., 3-C). HPLC-MS spectra: Figure S2; ${ }^{1} \mathrm{H}$ NMR spectrum: Figure S9.

\subsubsection{7- $N$-( $\beta$-Alanyl)amino-2-pentyl-3H-phenoxazin-3-one TFA salt 22b}

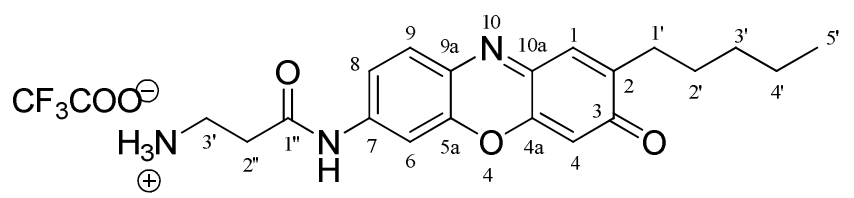

Prepared from 7- $N$-( $N$ '-tbutoxycarbonyl- $\beta$-alanyl)amino-2-pentyl-3 $H$-phenoxazin-3-one 20b $(0.14 \mathrm{~g}, 0.30 \mathrm{mmol}) .7-\mathrm{N}$-( $\beta$-Alanyl)amino-2-pentyl-3H-phenoxazin-3-one TFA salt 22b was isolated as a brown-red solid with a green metallic shine $(0.12 \mathrm{~g}, 0.27 \mathrm{mmol}, 88.5 \%)$; $\mathrm{mp} 213-$ $223^{\circ} \mathrm{C}$; HRMS (Found $(M)^{+}, 354.1815$. Calc. for $\mathrm{C}_{20} \mathrm{H}_{24} \mathrm{~N}_{3} \mathrm{O}_{3}$ : $(M)^{+}, 354.1812$ ); $\mathrm{m} / \mathrm{Z} 354.2$ $\left(M^{+}\right) v_{\max } / \mathrm{cm}^{-1} 3263(\mathrm{~N}-\mathrm{H}), 3188-3060\left(\mathrm{~N}^{+}-\mathrm{H}\right), 2928(\mathrm{C}-\mathrm{H}), 1674(\mathrm{C}=\mathrm{O}), 1651(\mathrm{C}=\mathrm{O}) ; \delta_{\mathrm{H}}$ (300 MHz, d 6 -DMSO) 0.88 (3H, t, $J=6.9 \mathrm{~Hz}, 5 '-\mathrm{H}), 1.30-1.33$ (4H, m, 3'-H and 4'-H), $1.485-$ $1.53\left(2 \mathrm{H}, \mathrm{m}, 2^{\prime}-\mathrm{H}\right), 2.45$ (2H, t, $\left.J=7.5 \mathrm{~Hz}, 1^{\prime}-\mathrm{H}\right), 2.82\left(2 \mathrm{H}, \mathrm{t}, J=6.3 \mathrm{~Hz}, 2^{\prime \prime}-\mathrm{H}\right), 3.15(2 \mathrm{H}, \mathrm{t}, J$ $=6.3 \mathrm{~Hz}, 3 "-\mathrm{H}), 6.25(1 \mathrm{H}, \mathrm{s}, 4-\mathrm{H}), 7.26(1 \mathrm{H}, \mathrm{s}, 1-\mathrm{H}), 7.50(1 \mathrm{H}, \mathrm{dd}, J=8.7,1.8 \mathrm{~Hz}, 8-\mathrm{H}), 7.73$ $(1 \mathrm{H}, \mathrm{d}, J=8.7 \mathrm{~Hz}, 9-\mathrm{H}), 7.89(1 \mathrm{H}, \mathrm{d}, J=1.8 \mathrm{~Hz}, 6-\mathrm{H}), 7.97\left(3 \mathrm{H}\right.$, br. s, $\left.\mathrm{NH}_{3}{ }^{+}\right), 10.83(1 \mathrm{H}, \mathrm{s}$, $\mathrm{NH}-\mathrm{Ar}) ; \delta_{\mathrm{C}}\left(75 \mathrm{MHz}, \mathrm{d}_{6}\right.$-DMSO) $14.4\left(\mathrm{CH}_{3}, 5^{\prime}-\mathrm{C}\right), 22.4\left(\mathrm{CH}_{2}, 3^{\prime}-\mathrm{C}\right.$ or 4'-C), $28.1\left(\mathrm{CH}_{2}, 2^{\prime}-\mathrm{C}\right)$, $29.4\left(\mathrm{CH}_{2}, 1^{\prime}-\mathrm{C}\right), 31.5\left(\mathrm{CH}_{2}, 3\right.$ '-H or 4'-H), $34.1\left(\mathrm{CH}_{2}, 2\right.$ "-C), $35.2\left(\mathrm{CH}_{2}, 3\right.$ "-C), $105.5(\mathrm{CH}, 6-$ C), 106.1 (CH, 4-C), 116.8 (CH, 8-C), 129.8 (quat., 7-C), 130.8 (quat., 9-C or 1-C), 130.9 (quat., 9-C or 1-C), 143.1 (quat., 9a-C), 144.4 (quat. 5a-C), 146.7 (quat., 2-C or 10a-C), 146.8 (quat., 2-C or 10a-C), 149.4 (quat., 4a-C), 169.7 (quat., 1"-C), 185.1 (quat., 3-C). 


\subsubsection{8- $N$-( $\beta$-Alanyl)amino-3H-phenoxazin-3-one TFA salt 23a}

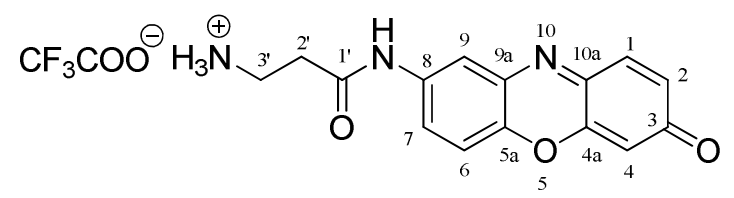

The title compound was prepared as described from $8-N-\left(N^{\prime}-\right.$ tbutoxycarbonyl- $\beta$-alanyl)amino$3 H$-phenoxazin-3-one 21a $(0.16 \mathrm{~g}, 0.43 \mathrm{mmol}) .8-\mathrm{N}$-( $\beta$-Alanyl)amino-3H-phenoxazin-3-one TFA salt 23a was isolated as a dark red solid $(0.14 \mathrm{~g}, 0.35 \mathrm{mmol}, 80.7 \%)$; mp $167-170^{\circ} \mathrm{C}$; HRMS (Found $(M)^{+}$, 284.1031. Calc. for $\left.\mathrm{C}_{15} \mathrm{H}_{14} \mathrm{~N}_{3} \mathrm{O}_{3}:(M)^{+}, 284.1030\right) ; m / Z 284.0(M)^{+}$; $v_{\max } / \mathrm{cm}^{-1} 3408-2907\left(\mathrm{~N}^{+}-\mathrm{H}\right), 3053(\mathrm{~N}-\mathrm{H}), 1671$ and $1643(\mathrm{C}=\mathrm{O}) ; \delta_{\mathrm{H}}\left(300 \mathrm{MHz}, \mathrm{d}_{6}-\mathrm{DMSO}\right)$ $2.77\left(2 \mathrm{H}, \mathrm{t}, J=6.6 \mathrm{~Hz}, 2^{\prime}-\mathrm{H}\right), 3.14(2 \mathrm{H}$, br. s, 3'-H), $6.245(1 \mathrm{H}, \mathrm{d}, J=1.8 \mathrm{~Hz}, 4-\mathrm{H}), 6.835(1 \mathrm{H}$, $\mathrm{dd}, J=9.9,1.5 \mathrm{~Hz}, 2-\mathrm{H}), 7.44(1 \mathrm{H}, \mathrm{d}, J=9.0 \mathrm{~Hz}, 6-\mathrm{H}), 7.52(1 \mathrm{H}, \mathrm{d}, J=9.9 \mathrm{~Hz}, 1-\mathrm{H}), 7.77(1 \mathrm{H}$, dd, $J=9.0,2.1 \mathrm{~Hz}, 7-\mathrm{H}), 7.98\left(3 \mathrm{H}\right.$, br. s, $\left.\mathrm{NH}_{3}{ }^{+}\right), 8.15(1 \mathrm{H}, \mathrm{d}, J=2.4 \mathrm{~Hz}, 9-\mathrm{H}), 10.56(1 \mathrm{H}, \mathrm{s}$, $\mathrm{NH}$-Ar); $\delta_{\mathrm{C}}\left(75 \mathrm{MHz}, \mathrm{d}_{6}\right.$-DMSO) $33.8\left(\mathrm{CH}_{2}, 2^{\prime}-\mathrm{C}\right), 35.4\left(\mathrm{CH}_{2}, 3^{\prime}-\mathrm{C}\right), 106.1(\mathrm{CH}, 4-\mathrm{C}), 116.7$ $(\mathrm{CH}, 6-\mathrm{C}), 119.4(\mathrm{CH}, 9-\mathrm{C}), 124.7$ (CH, 7-C), 133.3 (quat., 9a-C), $135.3(\mathrm{CH}, 1-\mathrm{C}$ and 2-C), 136.8 (quat., 8-C), 139.8 (quat., 5a-C), 149.2 (quat., 10a-C), 150.1 (quat., 4a-C), 169.05 (quat., 1'-C), 185.95 (quat., 3-C). ${ }^{1} \mathrm{H}$ NMR spectrum: Figure S11.

\subsubsection{8- $N$-( $\beta$-Alanyl)amino-1,2,4-trimethyl-3H-phenoxazin-3-one TFA salt 23b}

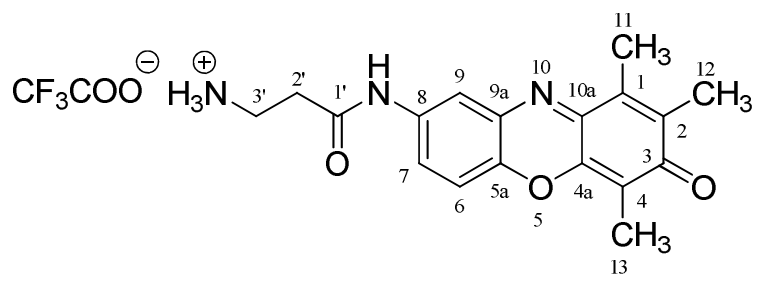

Prepared from 8- $N$ - $\left(N^{\prime}\right.$-'butoxycarbonyl- $\beta$-alanyl)amino-1,2,4-trimethyl-3H-phenoxazin-3-one 21b (0.06g, 0.16mmol). 8- $N$-( $\beta$-Alanyl)amino-1,2,4-trimethyl-3H-phenoxazin-3-one TFA salt 23b was isolated as a red solid (0.06g, 0.14mmol, 91.2\%); mp 231-235 ${ }^{\circ} \mathrm{C}$; HRMS (Found $(M)^{+}$, 326.1501. Calc. for $\left.\mathrm{C}_{18} \mathrm{H}_{20} \mathrm{~N}_{3} \mathrm{O}_{3}:(M)^{+}, 326.1499\right) ; \mathrm{m} / \mathrm{Z} 326.2\left(M^{+}\right) ; v_{\max } / \mathrm{cm}^{-1} 3322(\mathrm{~N}-$ $\mathrm{H}), 3054\left(\mathrm{~N}^{+}-\mathrm{H}\right), 1670$ and $1649(\mathrm{C}=\mathrm{O})$; $\delta_{\mathrm{H}}\left(300 \mathrm{MHz}, \mathrm{d}_{6}-\mathrm{DMSO}\right) 1.97(3 \mathrm{H}, \mathrm{s}, 13-\mathrm{H}), 2.07$ $(3 \mathrm{H}, \mathrm{s}, 12-\mathrm{H}), 2.33(3 \mathrm{H}, \mathrm{s}, 11-\mathrm{H}), 2.76\left(2 \mathrm{H}, \mathrm{t}, J=6.6 \mathrm{~Hz}, 2^{\prime}-\mathrm{H}\right), 3.15\left(2 \mathrm{H}, \mathrm{t}, J=6.3 \mathrm{~Hz}, 3^{\prime}-\mathrm{H}\right)$, $7.41(1 \mathrm{H}, \mathrm{d}, J=8.7 \mathrm{~Hz}, 6-\mathrm{H}), 7.64(1 \mathrm{H}, \mathrm{dd}, J=8.7,2.1 \mathrm{~Hz}, 7-\mathrm{H}), 7.855\left(3 \mathrm{H}\right.$, br. s, $\left.\mathrm{NH}_{3}{ }^{+}\right), 8.15$ $(1 \mathrm{H}, \mathrm{d}, J=2.1 \mathrm{~Hz}, 9-\mathrm{H}), 10.40(1 \mathrm{H}, \mathrm{s}, \mathrm{NH}-\mathrm{Ar}) ; \delta_{\mathrm{C}}\left(75 \mathrm{MHz}, \mathrm{d}_{6}-\mathrm{DMSO}\right) 7.9\left(\mathrm{CH}_{3}, 13-\mathrm{C}\right), 12.8$ $\left(\mathrm{CH}_{3}, 12-\mathrm{C}\right), 13.2\left(\mathrm{CH}_{3}, 11-\mathrm{C}\right), 33.9\left(\mathrm{CH}_{2}, 2^{\prime}-\mathrm{C}\right), 35.8\left(\mathrm{CH}_{2}, 3^{\prime}-\mathrm{C}\right), 113.2$ (quat., 4-C), 116.1 (CH, 6-C), 120.0 (CH, 9-C), 123.8 (CH, 7-C), 132.6 (quat., 9a-C), 136.2 (quat., 8-C), 136.9 
(quat., 1-C or 2-C), 139.0 (quat., 1-C or 2-C), 140.2 (quat., 5a-C), 145.5 (quat., 4a-C), 149.15 (quat., 10a-C), 169.0 (quat., 1'-C), 184.4 (quat., 3-C). HPLC-MS spectra: Figure S4; ${ }^{1} \mathrm{H}$ NMR spectrum: Figure S12.

\subsubsection{8- $N$-( $\beta$-Alanyl)amino-1,2-dimethyl-3H-phenoxazin-3-one TFA salt 23c}

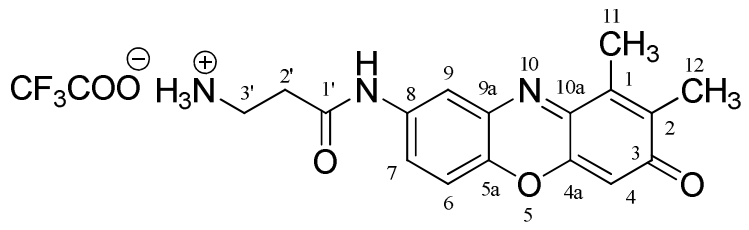

The title compound was prepared as described from $8-N-\left(N^{\prime}-\right.$ butoxycarbonyl- $\beta$-alanyl $)$ amino1,2-dimethyl-3H-phenoxazin-3-one $\quad 21 \mathrm{c} \quad(0.20 \mathrm{~g}, \quad 0.49 \mathrm{mmol}) . \quad 8-N$-( $\beta$-Alanyl)amino-1,2dimethyl-3H-phenoxazin-3-one TFA salt 23c was isolated as an orange-red solid $(0.20 \mathrm{~g}$, 0.47mmol, 96.1\%); mp 202-207 ${ }^{\circ} \mathrm{C}$; HRMS (Found $(M)^{+}$, 312.1344. Calc. for $\mathrm{C}_{17} \mathrm{H}_{18} \mathrm{~N}_{3} \mathrm{O}_{3}$ : $\left.(M)^{+}, 312.1343\right) ; m / z 312.2\left(M^{+}\right) ; v_{\max } / \mathrm{cm}^{-1} 3282(\mathrm{~N}-\mathrm{H}), 3082\left(\mathrm{~N}^{+}-\mathrm{H}\right), 1671$ and $1645(\mathrm{C}=\mathrm{O})$; $\delta_{\mathrm{H}}\left(300 \mathrm{MHz}, \mathrm{d}_{6}\right.$-DMSO) $2.00(3 \mathrm{H}, \mathrm{s}, 12-\mathrm{H}), 2.26(3 \mathrm{H}, \mathrm{s}, 11-\mathrm{H}), 2.77\left(2 \mathrm{H}, \mathrm{t}, J=6.6 \mathrm{~Hz}, 2^{\prime}-\right.$ H), $3.16(2 \mathrm{H}, \mathrm{t}, J=6.6 \mathrm{~Hz}, 3$ '-H), $6.08(1 \mathrm{H}, \mathrm{s}, 4-\mathrm{H}), 7.32(1 \mathrm{H}, \mathrm{d}, J=8.7 \mathrm{~Hz}, 6-\mathrm{H}), 7.60(1 \mathrm{H}$, dd, $J=8.7,1.8 \mathrm{~Hz}, 7-\mathrm{H}), 7.99\left(3 \mathrm{H}\right.$, br. s, $\left.\mathrm{NH}_{3}{ }^{+}\right), 8.11(1 \mathrm{H}, \mathrm{d}, J=1.8 \mathrm{~Hz}, 9-\mathrm{H}), 10.46(1 \mathrm{H}, \mathrm{s}$, $\mathrm{NH}-\mathrm{Ar}) ; \delta_{\mathrm{C}}\left(75 \mathrm{MHz}, \mathrm{d}_{6}\right.$-DMSO) $13.0\left(\mathrm{CH}_{3}, 11-\mathrm{C}\right.$ or $\left.12-\mathrm{C}\right), 13.05\left(\mathrm{CH}_{3}, 11-\mathrm{C}\right.$ or $\left.12-\mathrm{C}\right), 33.75$ $\left(\mathrm{CH}_{2}, 2\right.$ '-C), $35.4\left(\mathrm{CH}_{2}, 3^{\prime}-\mathrm{C}\right), 104.9$ (CH, 4-C), $116.25(\mathrm{CH}, 6-\mathrm{C}), 119.3(\mathrm{CH}, 9-\mathrm{C}), 123.7$ (CH, 7-C), 132.6 (quat., 9a-C), 136.5 (quat., 8-C), 137.7 (quat., 1-C or 2-C), 139.4 (quat., 5a$\mathrm{C}$ or 1-C or 2-C), 139.45 (quat., $5 \mathrm{a}-\mathrm{C}$ or $1-\mathrm{C}$ or 2-C), 148.9 (quat., $4 \mathrm{a}-\mathrm{C}$ or 10a-C), 149.2 (quat., 4a-C or 10a-C), 168.9 (quat., 1'-C), 184.7 (quat., 3-C). HPLC-MS spectra: Figure S5; ${ }^{1}$ H NMR spectrum: Figure S13.

\subsubsection{8- $N$-( $\beta$-Alanyl)amino-2- ${ }^{t}$ butyl-3H-phenoxazin-3-one TFA salt $23 d$}

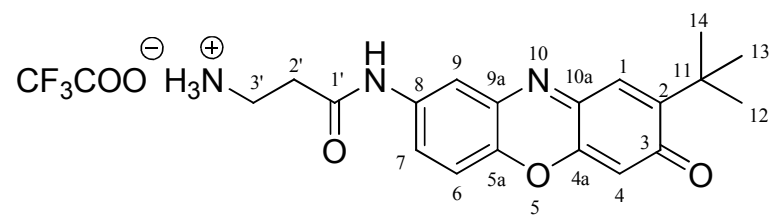

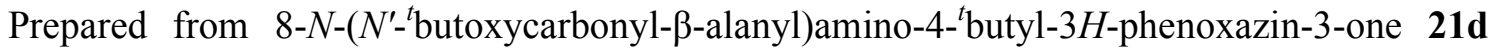
$(0.16 \mathrm{~g}, 0.37 \mathrm{mmol}) .8-N$-( $\beta$-Alanyl)amino-4-butyl-3H-phenoxazin-3-one TFA salt 23d was obtained as an orange-brown solid $(0.16 \mathrm{~g}, 0.35 \mathrm{mmol}, 93.5 \%)$; mp $119-126^{\circ} \mathrm{C}$; HRMS (Found $(M)^{+}, 340.1657$. Calc. for $\left.\mathrm{C}_{19} \mathrm{H}_{22} \mathrm{~N}_{3} \mathrm{O}_{3}:(M)^{+}, 340.1656\right) ; \mathrm{m} / \mathrm{Z} 340.2\left(M^{+}\right) ; v_{\max } / \mathrm{cm}^{-1} 3462(\mathrm{~N}-$ $\mathrm{H}), 3308-2965\left(\mathrm{~N}^{+}-\mathrm{H}\right)$ and $(\mathrm{C}-\mathrm{H}), 1678(\mathrm{C}=\mathrm{O}), 1649(\mathrm{C}=\mathrm{O}) ; \delta_{\mathrm{H}}\left(300 \mathrm{MHz}, \mathrm{d}_{6}-\mathrm{DMSO}\right) 1.32$ 
(9H, s, C(CH3) $\left.)_{3}\right), 2.78\left(2 \mathrm{H}, \mathrm{t}, J=6.6 \mathrm{~Hz}, 2^{\prime}-\mathrm{H}\right), 3.30(2 \mathrm{H}$, br. s, 3'-H), $6.15(1 \mathrm{H}, \mathrm{s}, 4-\mathrm{H}), 7.225$ $(1 \mathrm{H}, \mathrm{s}, 1-\mathrm{H}), 7.42(1 \mathrm{H}, \mathrm{d}, J=9.0 \mathrm{~Hz}, 6-\mathrm{H}), 7.74(1 \mathrm{H}, \mathrm{dd}, J=9.0,1.8 \mathrm{~Hz}, 7-\mathrm{H}), 7.97(3 \mathrm{H}$, br. s, $\left.\mathrm{NH}_{3}{ }^{+}\right), 8.13(1 \mathrm{H}, \mathrm{d}, J=1.8 \mathrm{~Hz}, 9-\mathrm{H}) ; \delta_{\mathrm{C}}\left(75 \mathrm{MHz}, \mathrm{d}_{6}-\mathrm{DMSO}\right) 29.4\left(\mathrm{CH}_{3}, 12 / 13 / 14-\mathrm{C}\left(\mathrm{CH}_{3}\right)_{3}\right)$, $33.8\left(\mathrm{CH}_{2}, 2^{\prime}-\mathrm{C}\right), 35.4$ (quat., 11-C( $\left.\left(\mathrm{CH}_{3}\right)_{3}\right), 35.7\left(\mathrm{CH}_{2}, 3\right.$ '-C), $107.4(\mathrm{CH}, 4-\mathrm{C}), 116.6(\mathrm{CH}, 6-$ C), 119.3 (CH, 9-C), 124.2 (CH, 1-C), 129.8 (CH, 7-C), 133.5 (quat., 9a-C), 136.6 (quat., 8C), 139.5 (quat., 5a-C), 148.65 (quat., 4a-C or 10a-C), 149.6 (quat., 4a-C or 10a-C), 154.1 (quat., 2-C), 169.0 (quat., 1'-C), 183.2 (quat., 3-C). HPLC-MS spectra: Figure S6; ${ }^{1} \mathrm{H}$ NMR spectrum: Figure S14.

\subsubsection{8- $N$-( $\beta$-alanyl)amino-2-pentyl-3H-phenoxazin-3-one TFA salt 23e}

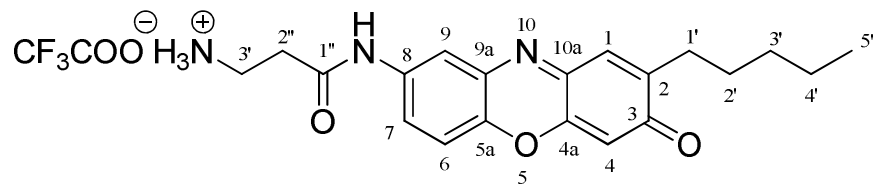

Prepared from 8- $N$-( $N^{\prime}$-'butoxycarbonyl- $\beta$-alanyl)amino-2-pentyl-3H-phenoxazin-3-one 21e $(0.08 \mathrm{~g}, 0.18 \mathrm{mmol}) .8-N$-( $\beta$-Alanyl)amino-2-pentyl-3H-phenoxazin-3-one TFA salt 23e was isolated as a dark red solid $(0.07 \mathrm{~g}, 0.15 \mathrm{mmol}, 87.0 \%)$; mp $132-139^{\circ} \mathrm{C}$; HRMS (Found $(M)^{+}$, 354.1813. Calc. for $\left.\mathrm{C}_{20} \mathrm{H}_{24} \mathrm{~N}_{3} \mathrm{O}_{3}:(M)^{+}, 354.1812\right) ; \mathrm{m} / \mathrm{z} 354.2\left(M^{+}\right) ; v_{\max } / \mathrm{cm}^{-1} 3312(\mathrm{~N}-\mathrm{H})$, $3077\left(\mathrm{~N}^{+}-\mathrm{H}\right), 2932-2861(\mathrm{C}-\mathrm{H}), 1672(\mathrm{C}=\mathrm{O}), 1649(\mathrm{C}=\mathrm{O}) ; \delta_{\mathrm{H}}\left(300 \mathrm{MHz}, \mathrm{d}_{6}-\mathrm{DMSO}\right) 0.80$ $\left(3 \mathrm{H}, \mathrm{t}, J=6.6 \mathrm{~Hz}, 5^{\prime}-\mathrm{H}\right), 1.22-1.25\left(4 \mathrm{H}, \mathrm{m}, 3^{\prime}-\mathrm{H}\right.$ and $\left.4^{\prime}-\mathrm{H}\right), 1.425-1.47\left(2 \mathrm{H}, \mathrm{m}, 2^{\prime}-\mathrm{H}\right), 2.41$ (2H, t, $\left.J=8.1 \mathrm{~Hz}, 1^{\prime}-\mathrm{H}\right), 2.67(2 \mathrm{H}, \mathrm{t}, J=6.6 \mathrm{~Hz}, 2 "-\mathrm{H}), 3.05(2 \mathrm{H}$, br. s, 3"-H), $6.21(1 \mathrm{H}, \mathrm{s}, 4-\mathrm{H})$, $7.27(1 \mathrm{H}, \mathrm{s}, 1-\mathrm{H}), 7.40(1 \mathrm{H}, \mathrm{d}, J=9.0 \mathrm{~Hz}, 6-\mathrm{H}), 7.65(1 \mathrm{H}, \mathrm{dd}, J=9.0,2.4 \mathrm{~Hz}, 7-\mathrm{H}), 7.75(3 \mathrm{H}$, br. s, $\left.\mathrm{NH}_{3}{ }^{+}\right), 8.08(1 \mathrm{H}, \mathrm{d}, J=2.4 \mathrm{~Hz}, 9-\mathrm{H}), 10.39(1 \mathrm{H}, \mathrm{s}, \mathrm{NH}-\mathrm{Ar}) ; \delta_{\mathrm{C}}\left(75 \mathrm{MHz}, \mathrm{d}_{6}-\mathrm{DMSO}\right)$ $14.3\left(\mathrm{CH}_{3}, 5^{\prime}-\mathrm{C}\right), 22.4\left(\mathrm{CH}_{2}, 3^{\prime}-\mathrm{C}\right.$ or $\left.\quad 4 '-\mathrm{C}\right), 28.1\left(\mathrm{CH}_{2}, 2^{\prime}-\mathrm{C}\right), 29.4\left(\mathrm{CH}_{2}, 1 '-\mathrm{C}\right), 31.5\left(\mathrm{CH}_{2}, 3^{\prime}-\right.$ C or 4'-C), 33.9 ( $\left.\mathrm{CH}_{2}, 2^{\prime \prime}-\mathrm{C}\right), 35.4\left(\mathrm{CH}_{2}, 3\right.$ "-C), $105.9(\mathrm{CH}, 4-\mathrm{C}), 116.7(\mathrm{CH}, 6-\mathrm{C}), 119.2(\mathrm{CH}$, 9-C), 124.1 (CH, 7-C), 130.9 (CH, 1-C), 133.4 (quat., 9a-C), 136.7 (quat., 8-C), 139.6 (quat., 5a-C), 147.75 (quat., 2-C), 149.4 (quat., 4a-C or 10a-C), 149.5 (quat., 4a-C or 10a-C), 169.0 (quat., 1"-C), 185.2 (quat., 3-C).

\subsubsection{8- $N$-( $\beta$-Alanyl)amino-1-phenyl-3H-phenoxazin-3-one TFA salt $23 f$}

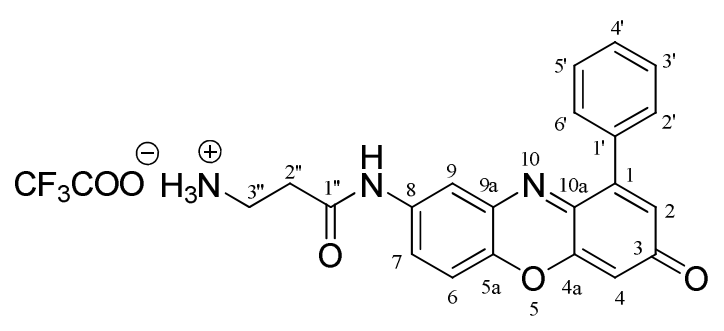


Prepared from 8- $N$-( $N^{\prime}$-'butoxycarbonyl- $\beta$-alanyl)amino-1-phenyl-3 $H$-phenoxazin-3-one 21f $(0.05 \mathrm{~g}, 0.12 \mathrm{mmol}) .8-\mathrm{N}$-( $\beta$-Alanyl)amino-1-phenyl-3H-phenoxazin-3-one TFA salt 23f was isolated as a dark red solid (0.05g, 0.10mmol, 82.2\%); mp 236-243 ${ }^{\circ} \mathrm{C}$; HRMS (Found $(M)^{+}$, 360.1345. Calc. for $\left.\mathrm{C}_{21} \mathrm{H}_{18} \mathrm{~N}_{3} \mathrm{O}_{3}:(M)^{+}, 360.1343\right) ; \mathrm{m} / \mathrm{Z} 360.2(M)^{+} ; v_{\max } / \mathrm{cm}^{-1} 3262(\mathrm{~N}-\mathrm{H})$, $3068\left(\mathrm{~N}^{+}-\mathrm{H}\right), 2903(\mathrm{C}-\mathrm{H}), 1670(\mathrm{C}=\mathrm{O}), 1640(\mathrm{C}=\mathrm{O}) ; \delta_{\mathrm{H}}\left(300 \mathrm{MHz}, \mathrm{d}_{6}-\mathrm{DMSO}\right) 2.67(2 \mathrm{H}, \mathrm{t}, J$ $=6.3 \mathrm{~Hz}, 2 "-\mathrm{H}), 3.05(2 \mathrm{H}, \mathrm{t}, J=6.3 \mathrm{~Hz}, 3 "-\mathrm{H}), 6.23(1 \mathrm{H}, \mathrm{d}, J=1.5 \mathrm{~Hz}, 4-\mathrm{H}), 6.72(1 \mathrm{H}, \mathrm{d}, J=$ 1.5Hz, 2-H), 7.37-7.42 (4H, m, 3'-H, 4'-H, 5'-H and 6-H), 7.52-7.53 (2H, m, 2'-H and 6'-H), $7.62(1 \mathrm{H}, \mathrm{dd}, J=8.7,1.8 \mathrm{~Hz}, 7-\mathrm{H}), 7.80\left(3 \mathrm{H}, \mathrm{s}, \mathrm{N} H_{3}{ }^{+}\right), 8.03(1 \mathrm{H}, \mathrm{d}, J=1.2 \mathrm{~Hz}, 9-\mathrm{H}), 10.33$ (1H, s, NH-Ar); $\delta_{\mathrm{C}}\left(75 \mathrm{MHz}, \mathrm{d}_{6}\right.$-DMSO) $33.8\left(\mathrm{CH}_{2}, 2 "-\mathrm{C}\right), 35.5\left(\mathrm{CH}_{2}, 3 "-\mathrm{C}\right), 106.1(\mathrm{CH}, 4-$

C), 116.5 (CH, 6-C), $120.0(\mathrm{CH}, 9-\mathrm{C}), 125.0(\mathrm{CH}, 7-\mathrm{C}), 128.35(\mathrm{CH}, 3$ '-C and 5'-C), 129.2 (CH, 4'-C), 130.5 (CH, 2'-C and 6'-C), 133.0 (quat., 9a-C), 135.2 (quat., 2-C), 136.6 (quat., 1C), 139.7 (quat., 8-C), 145.3 (quat., 5a-C), 148.4 (quat., 10a-C), 150.35 (quat., 4a-C), 169.1 (quat., 1"-C), 184.9 (quat., 3-C). HPLC-MS spectra: Figure S8; ${ }^{1} \mathrm{H}$ NMR spectrum: Figure S16.

\subsection{References}

1. Zaytsev, A.V.; Anderson, R.J.; Bedernjak, A.L.; Groundwater, P.W.; Huang, Y.; Perry, J.D.; Orenga, S.; Roger-Dalbert, C.; James, A.L. Org. Biomol. Chem., 2008, 6, 682.

2. Smith, J. Org. Chem. 1972, 37, 3972-3973.

3. Gwilherm, E.; Schaus, J.V.; Panek J.S. Org. Lett. 2004, 6, 525-528.

4. Yonezawa, S.; Komurasaki, T.; Kawada, K.; Tsuri, T.; Fuji, M.; Kugimiya, A.; Haga, N.; Mitsumori, S.; Inagaki, M.; Nakatani, T.; Tamura, Y.; Takechi, S.; Taishi, T.; Ohtani, M. J. Org. Chem. 1998, 63, 5831-5837.

5. Nakao, H.; Arakawa, M.; Nakamura, T. ; Fukushima, M. Chem. Pharm. Bull., 1972, 20, 1968.

6. Shulgin, A.T.; Dyer, D.C. J. Med. Chem., 1975, 18, 1201.

7. Liedholm, B. Acta Chem. Scand. Ser. B, 1976, 30, 141.

8. Behrman, E.C.; Chen, S. E.; Behrman, J. Tetrahedron Lett., 2002, 43, 3221. 
1.14 Figures: Selected LC-MS and NMR spectra of key intermediates and products

Figure S1. HPLC-MS Spectra of $\mathbf{2 b}$.

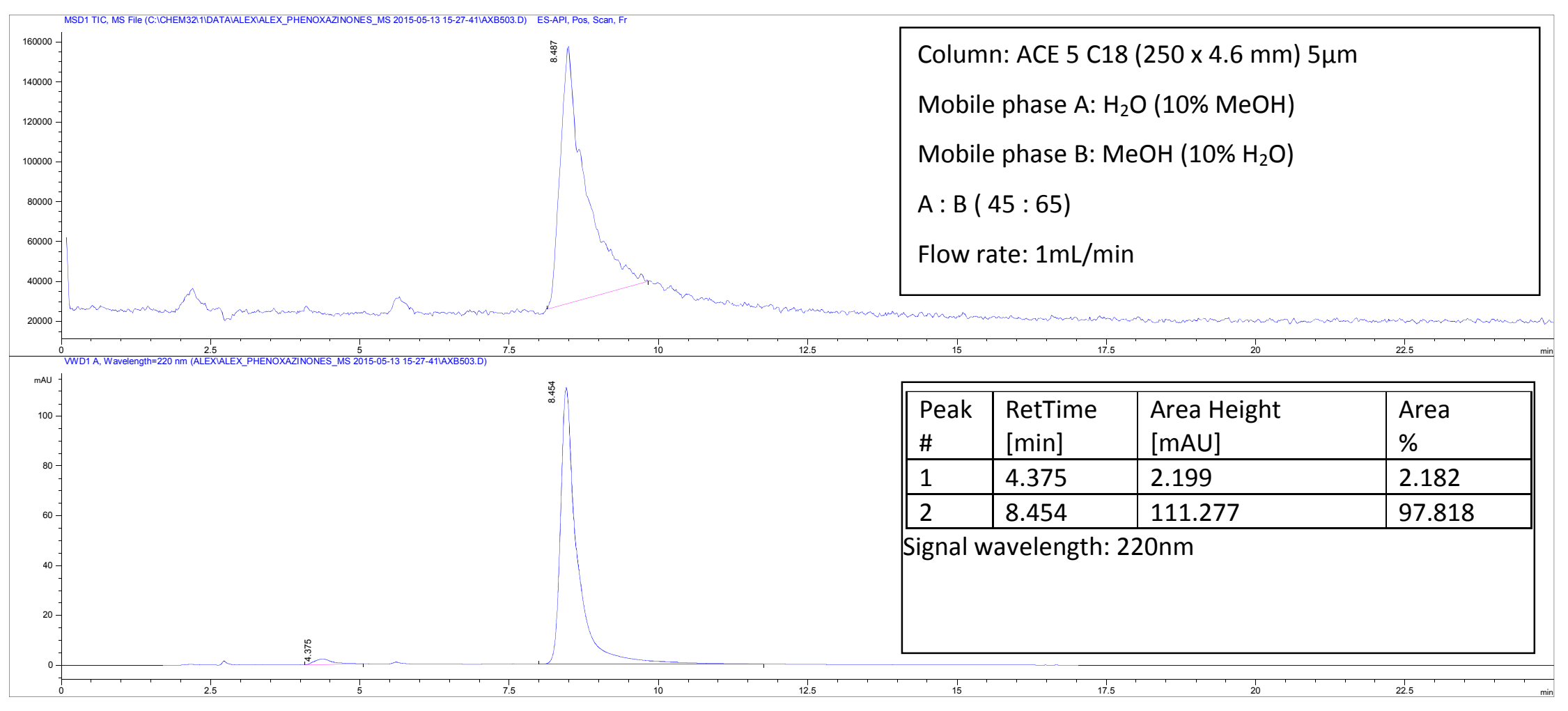




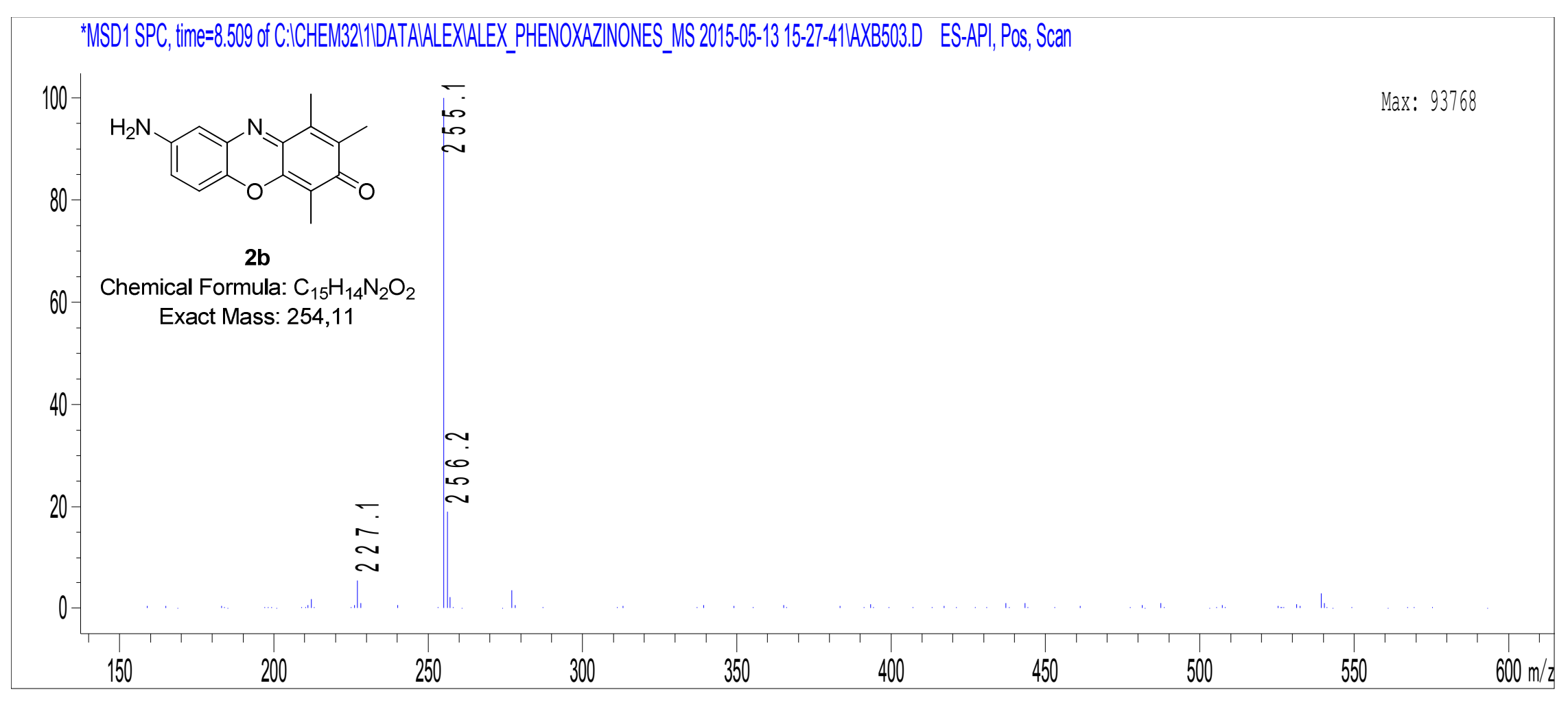


Figure S2. HPLC-MS Spectra of 2c.

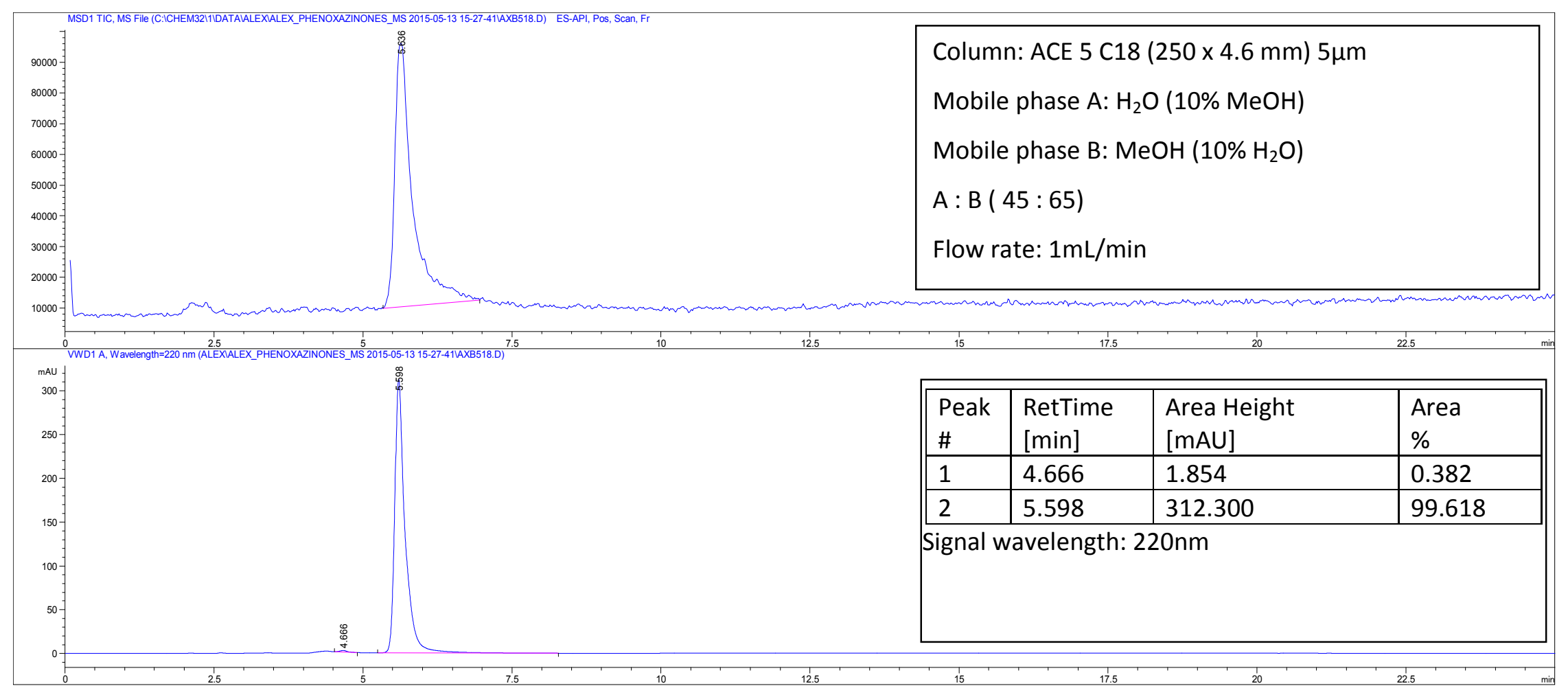




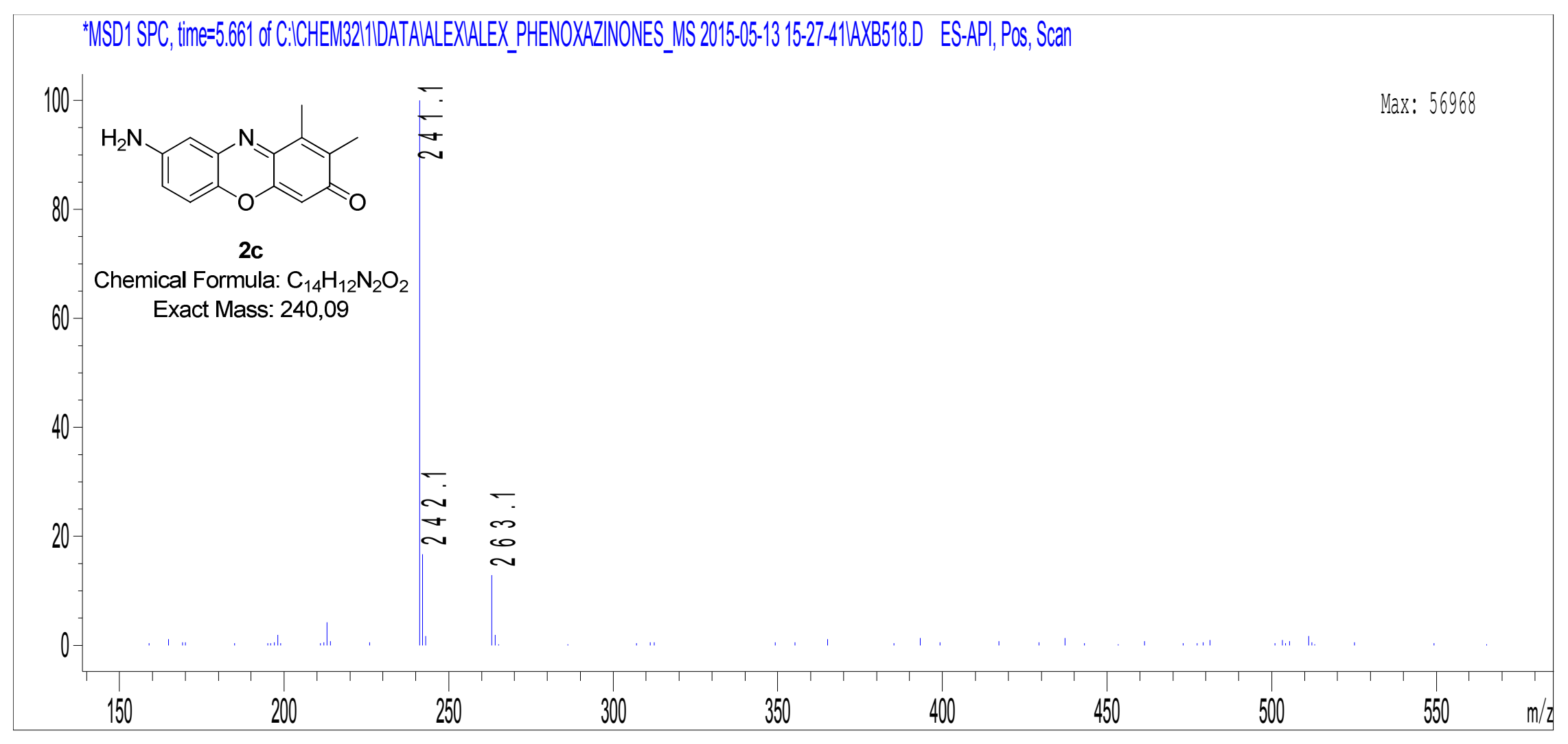


Figure S3. HPLC-MS Spectra of 22a.

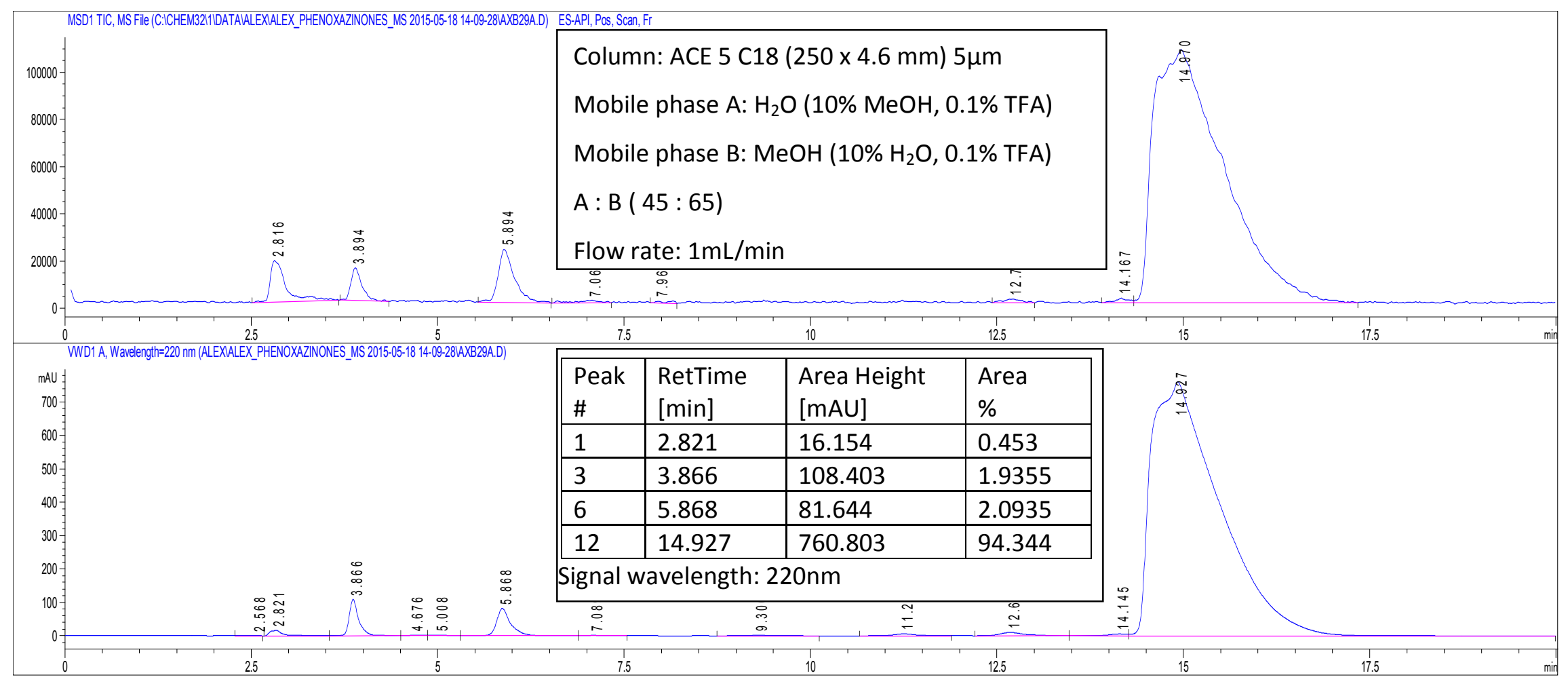




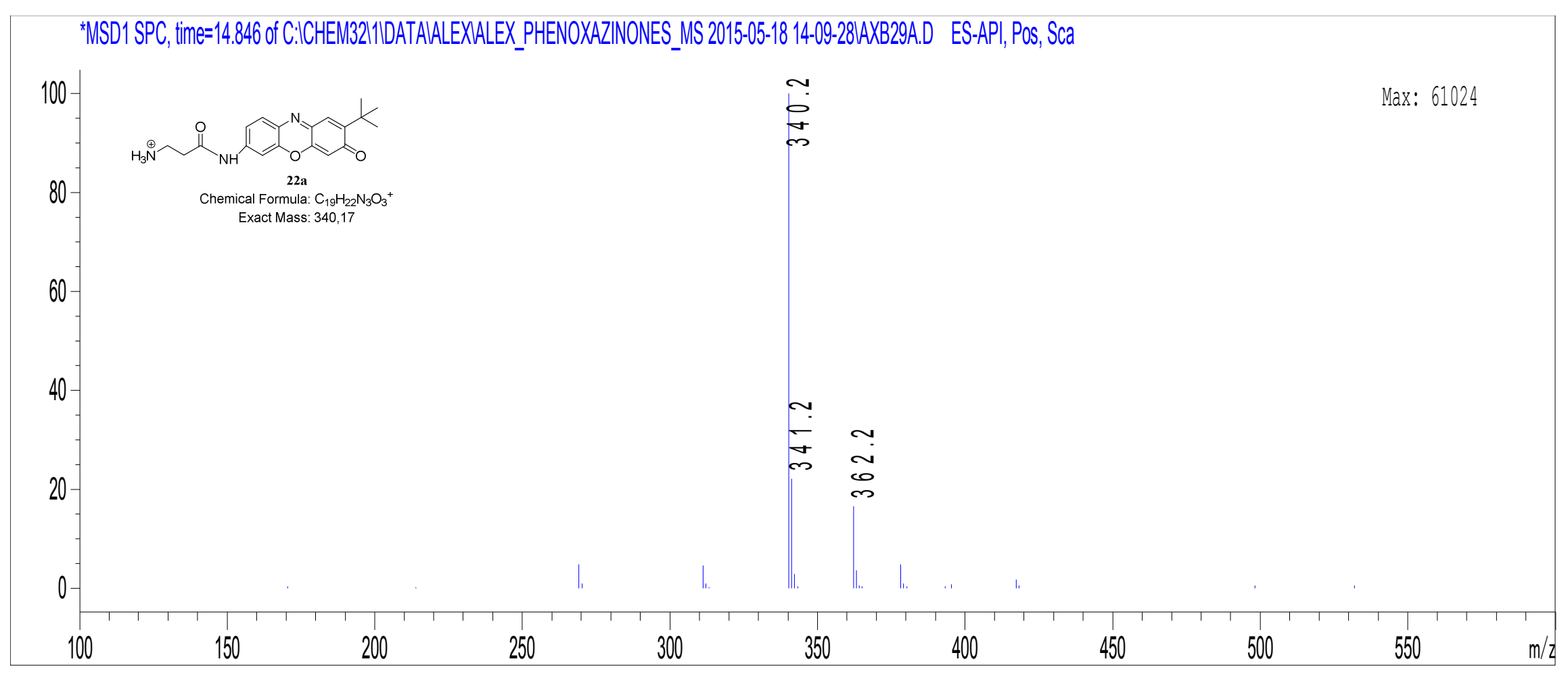


Figure S4. HPLC-MS Spectra of 23b.

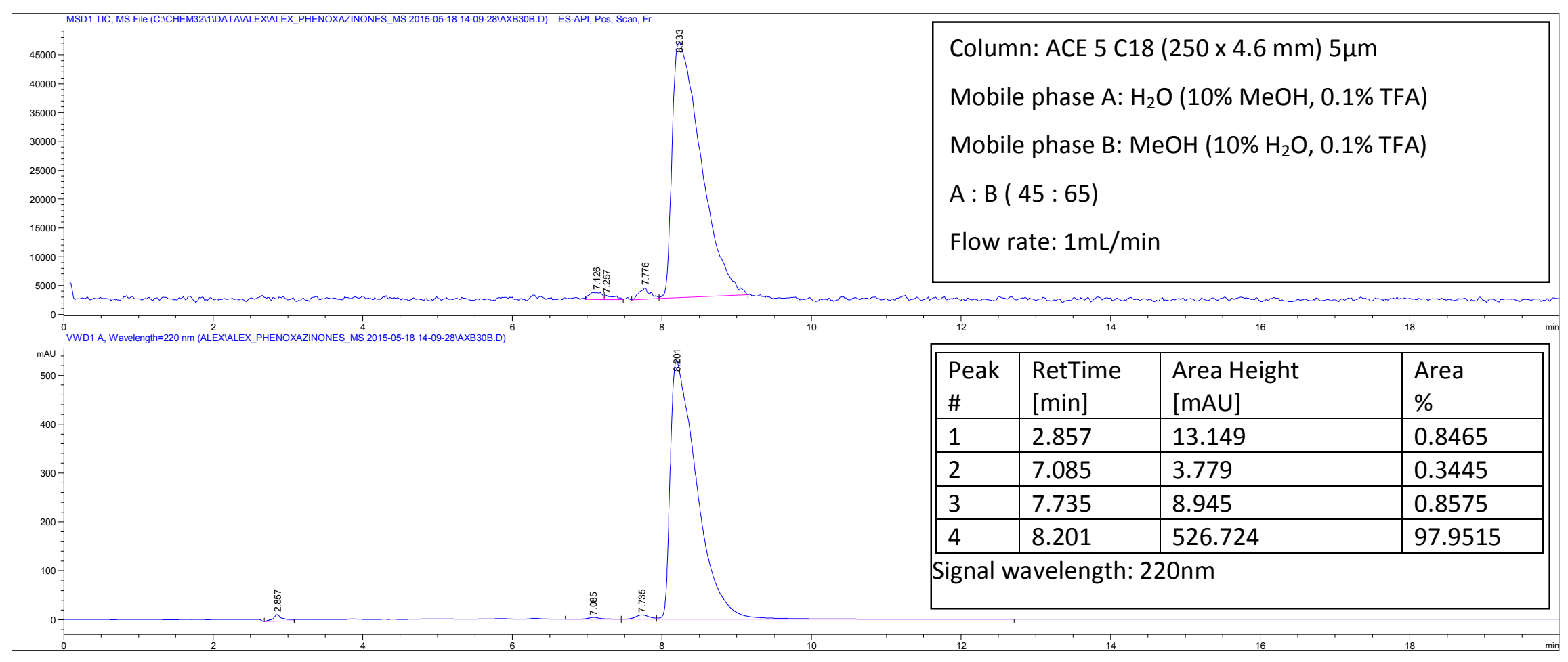




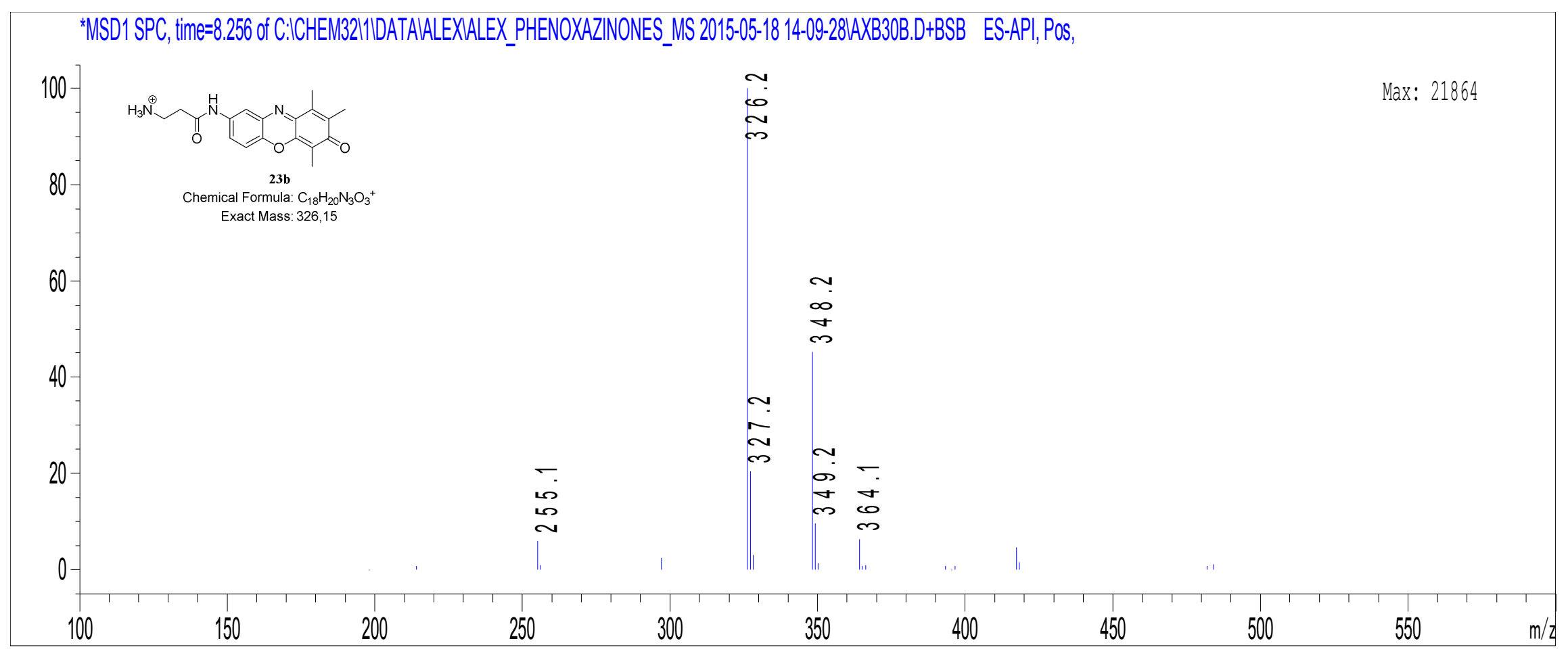


Figure S5. HPLC-MS Spectra of 23c.

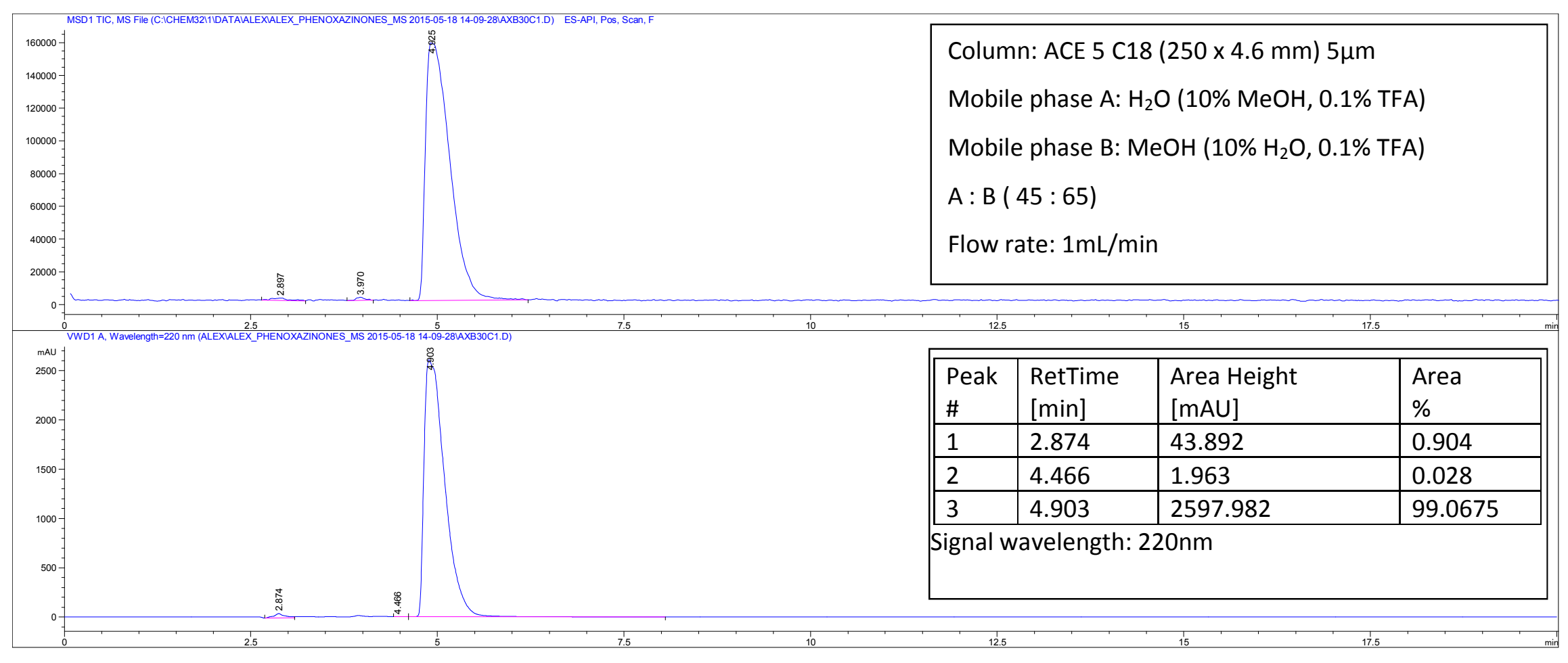




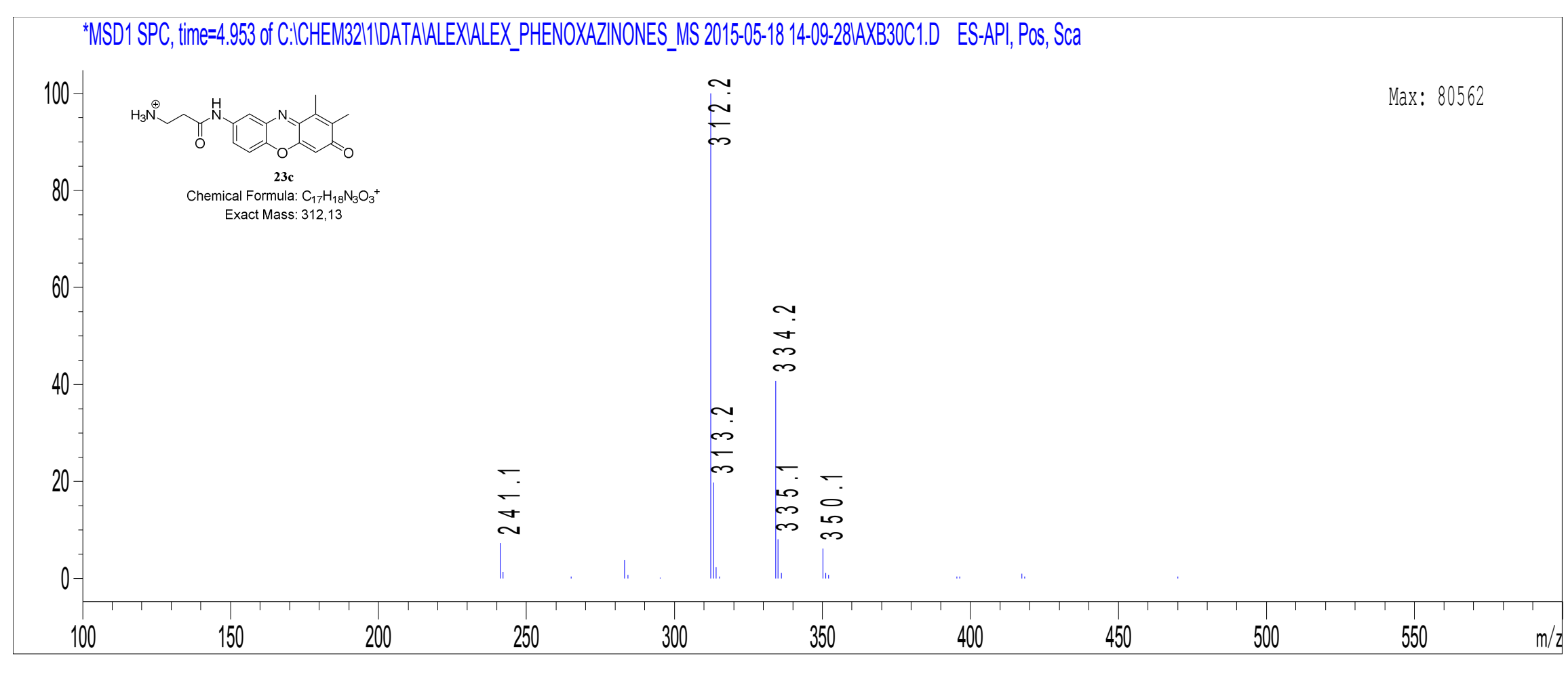


Figure S6. HPLC-MS Spectra of 23d.

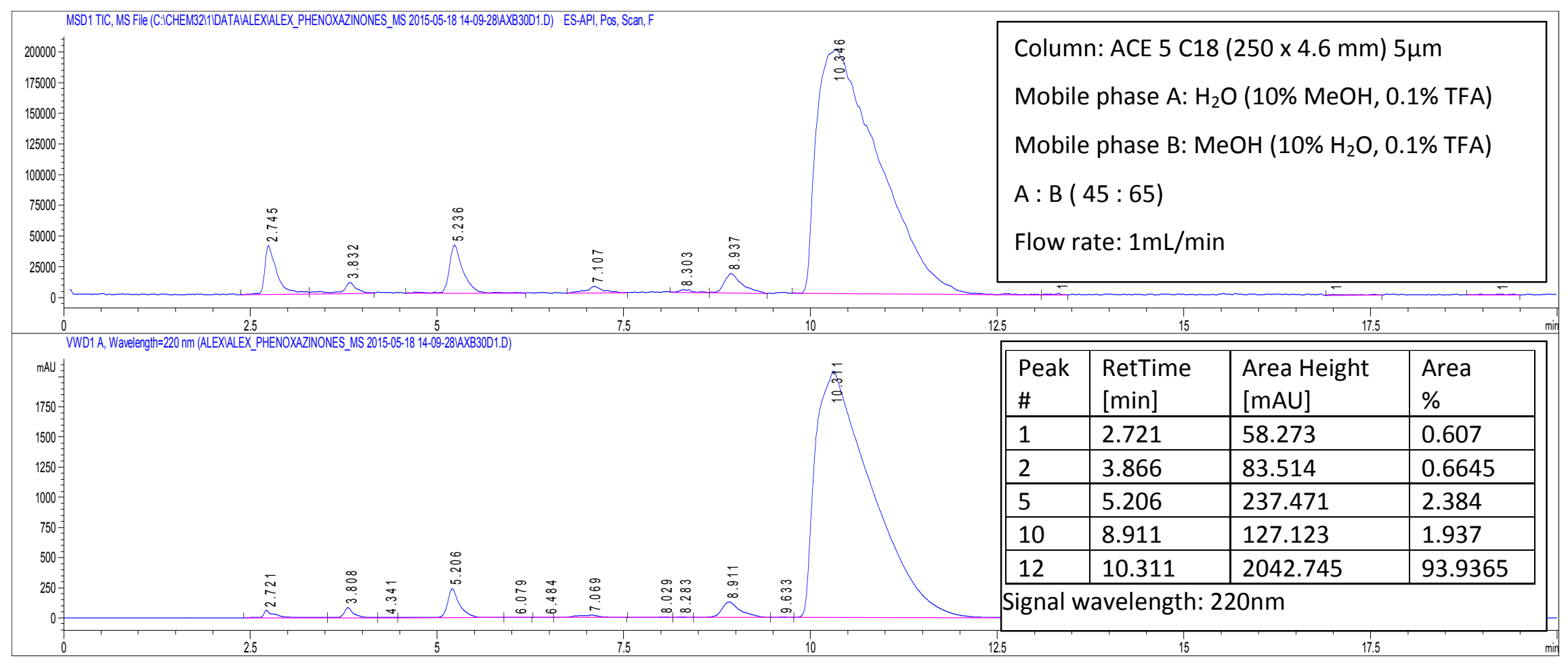




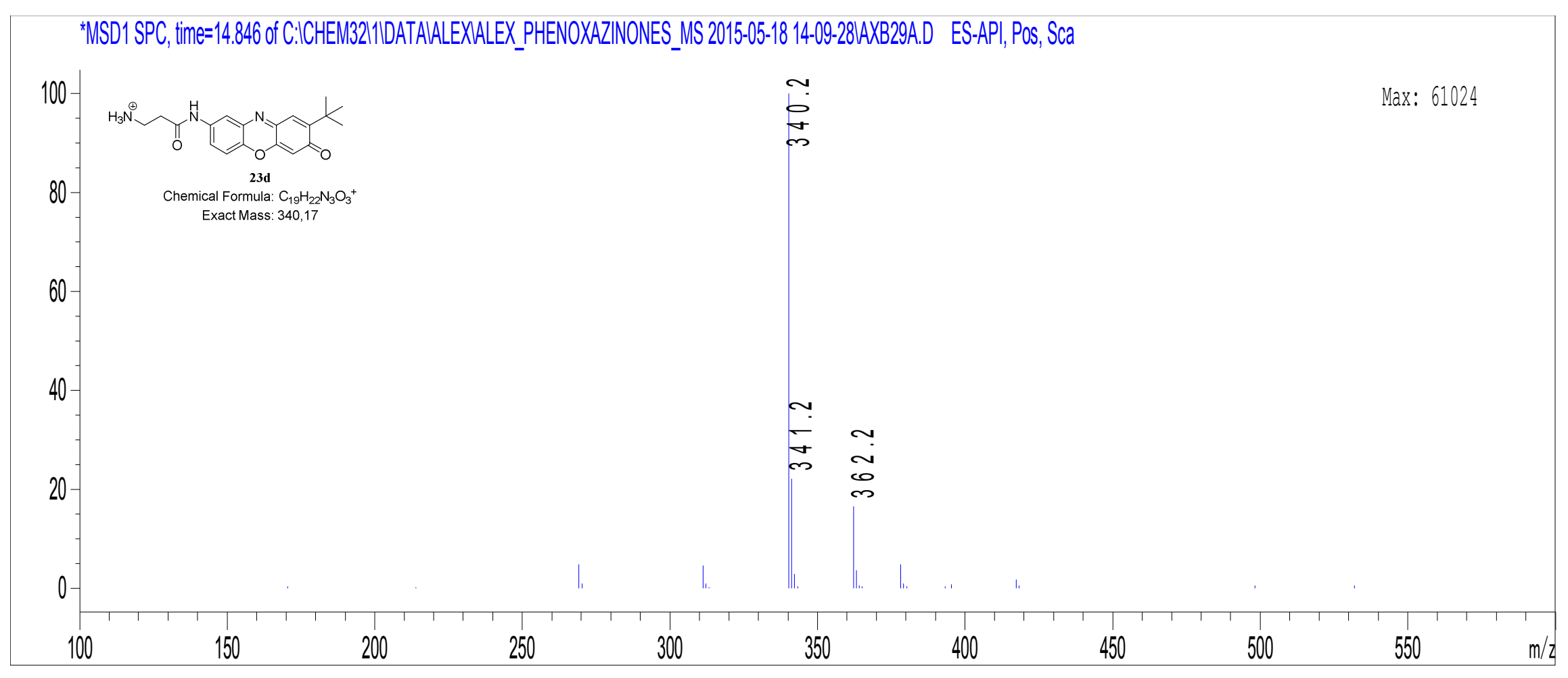


Figure S7. HPLC-MS Spectra of 23e.

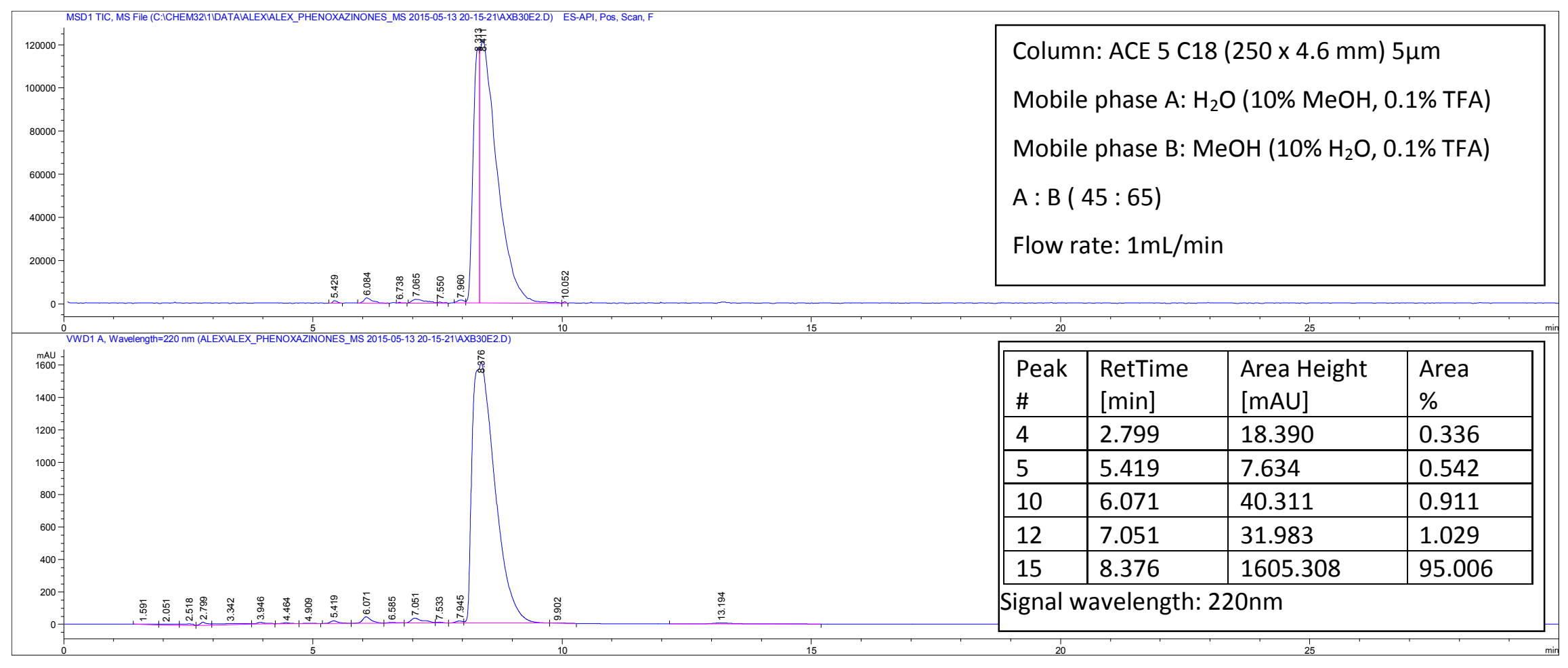




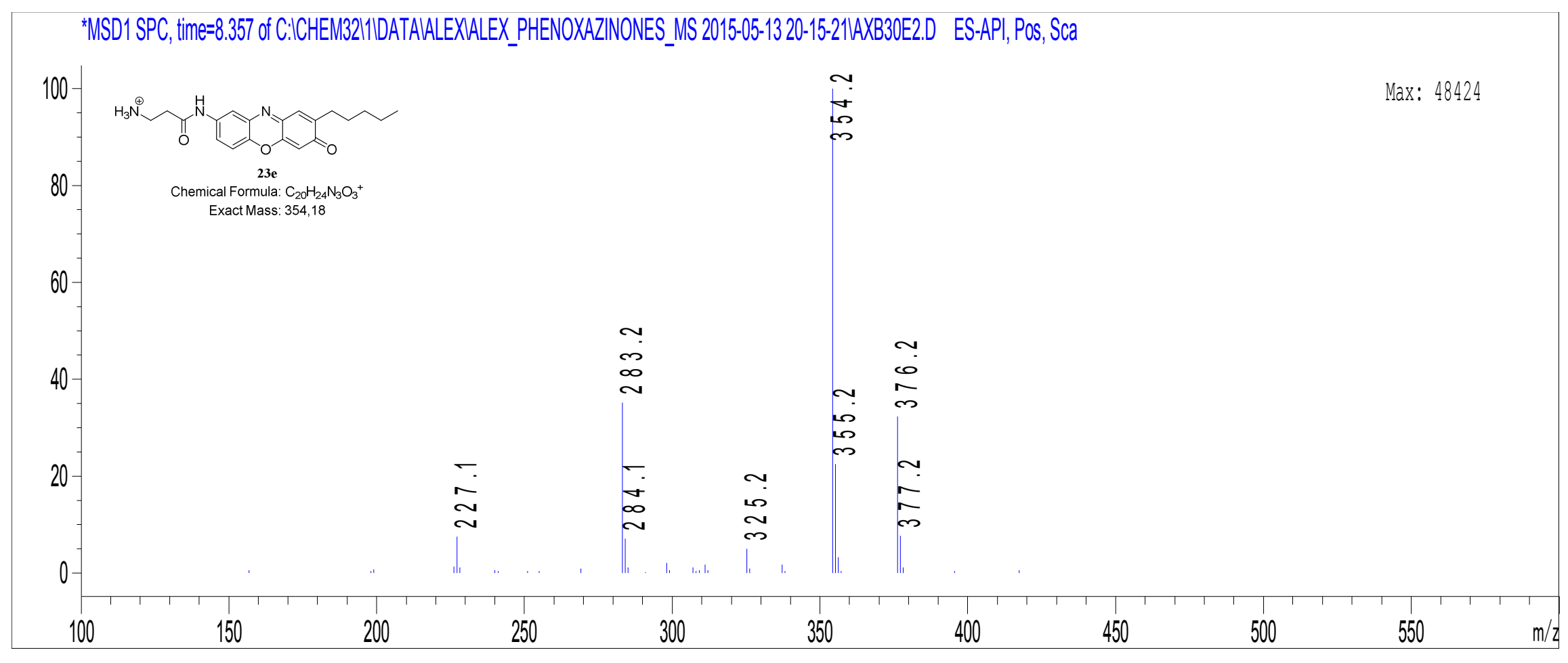


Figure S8. HPLC-MS Spectra of $23 f$.

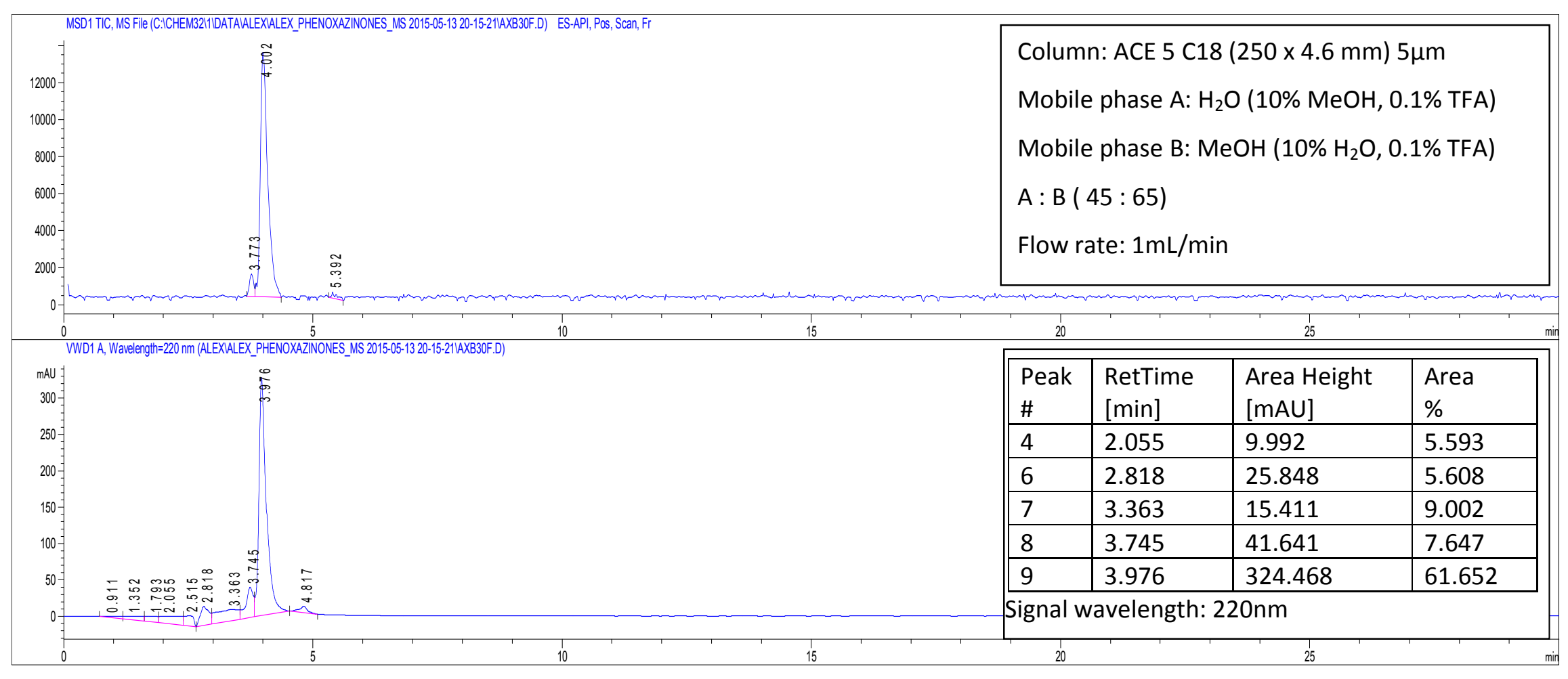




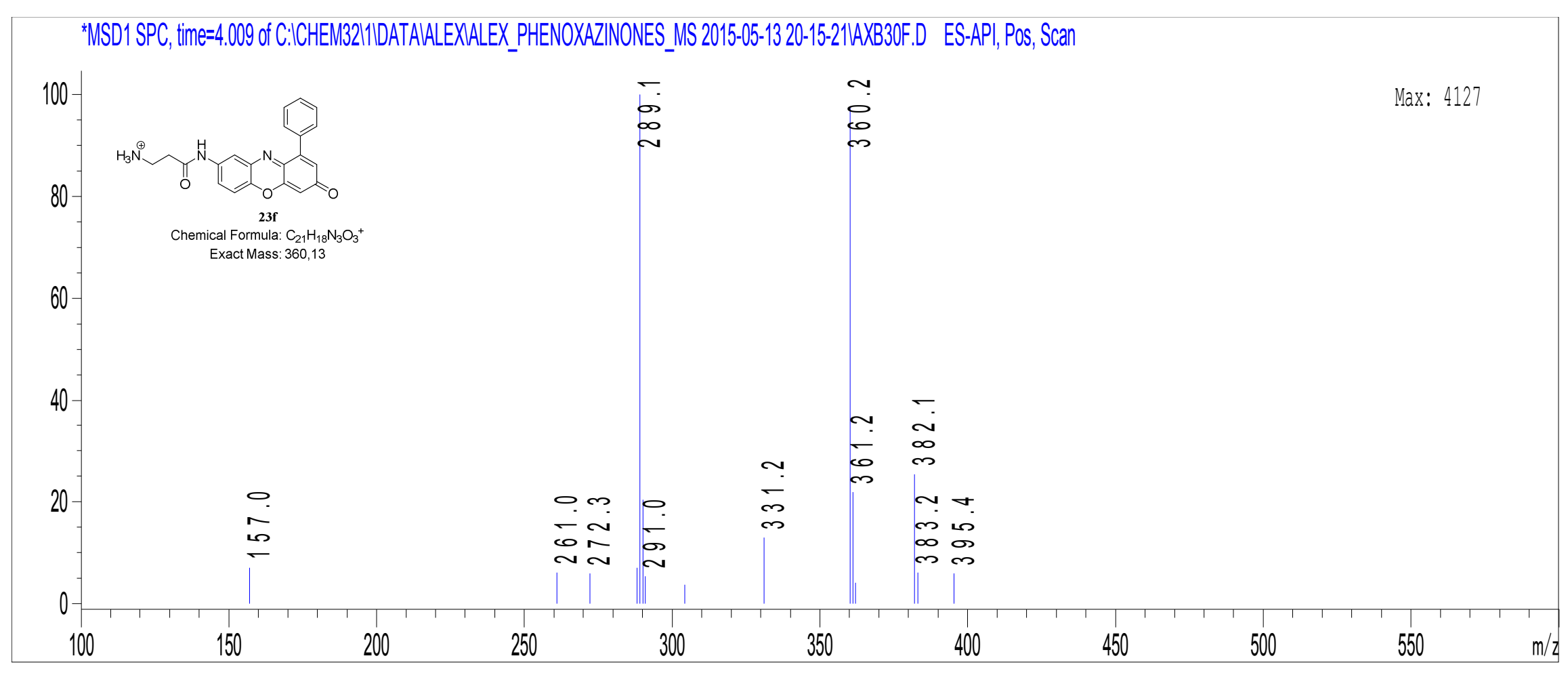


Figure S9. ${ }^{1} \mathrm{H}$ NMR spectrum of 22a in $\mathrm{d}_{6}$-DMSO at $50^{\circ} \mathrm{C}$.

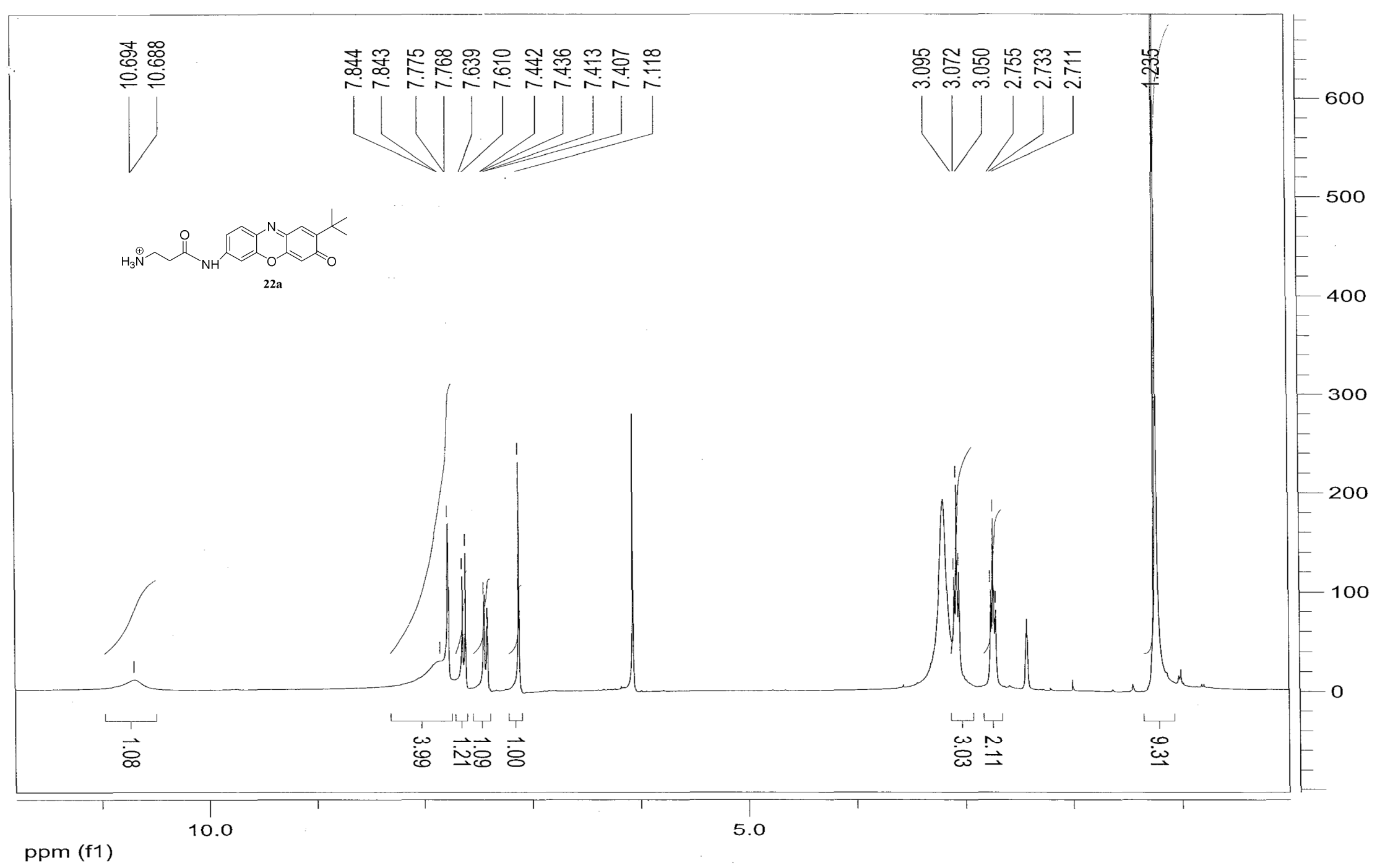




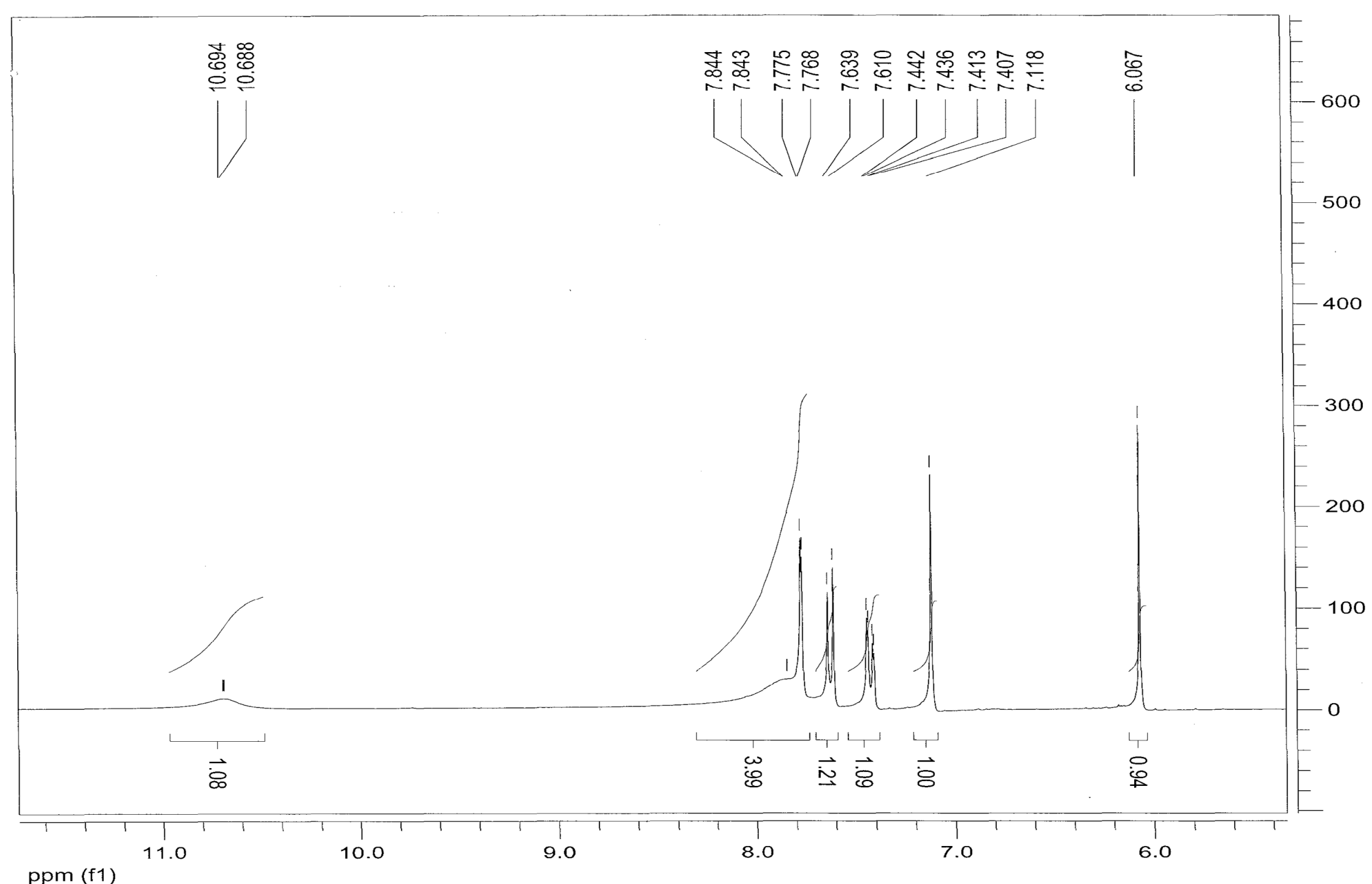




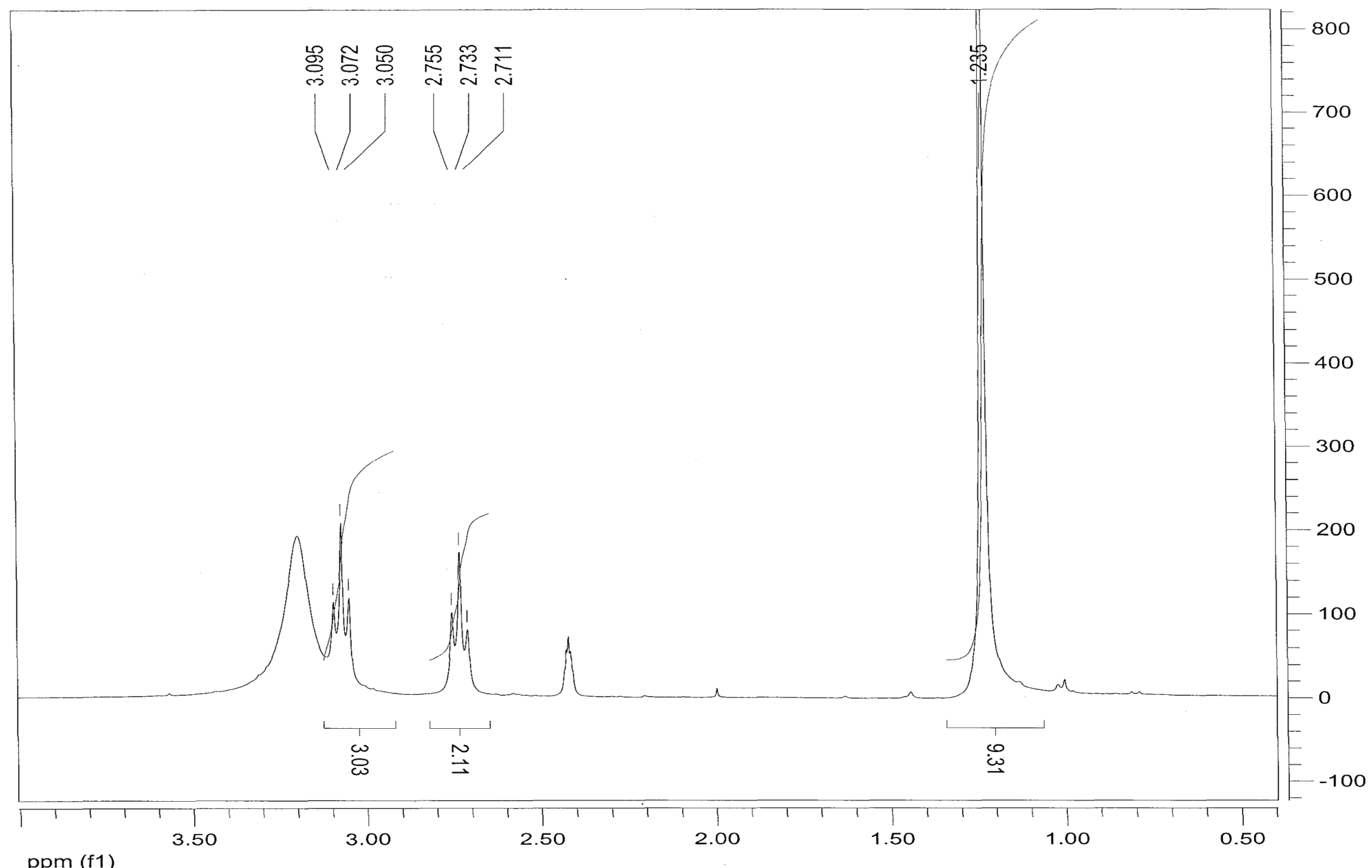


Figure S10. ${ }^{1} \mathrm{H}$ NMR spectrum of $\mathbf{2 2 b}$ in $\mathrm{d}_{6}$-DMSO at $50^{\circ} \mathrm{C}$.

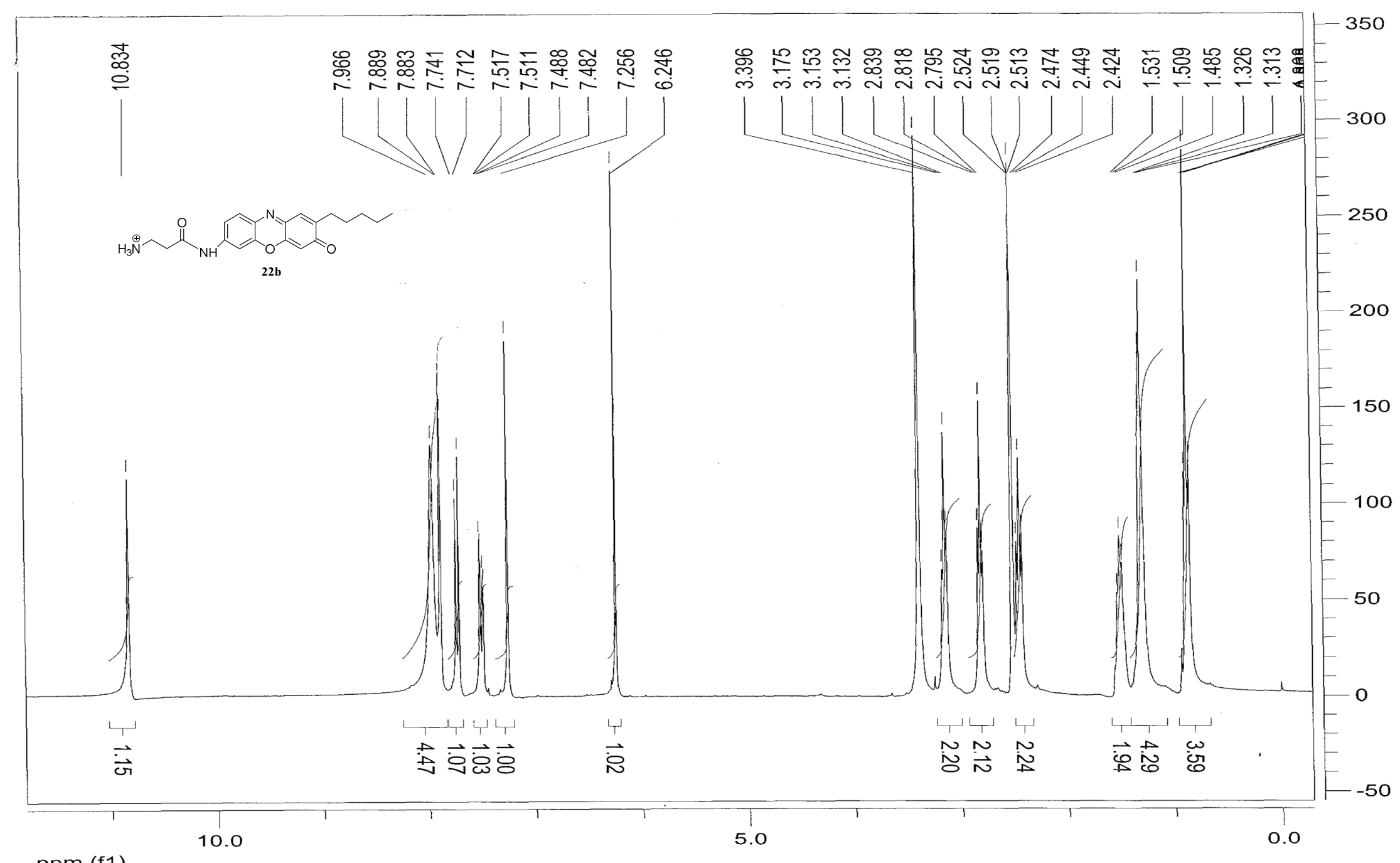




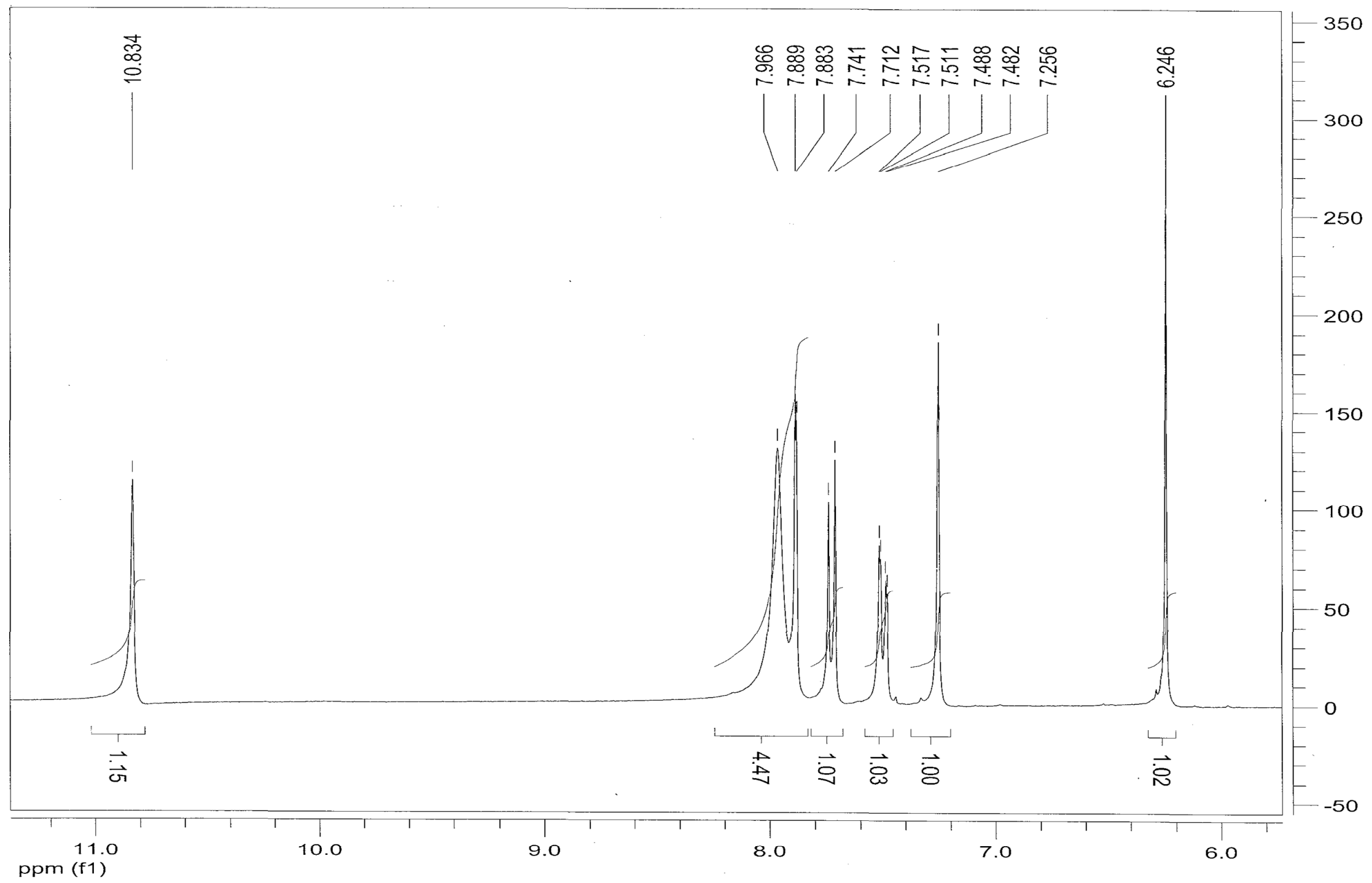




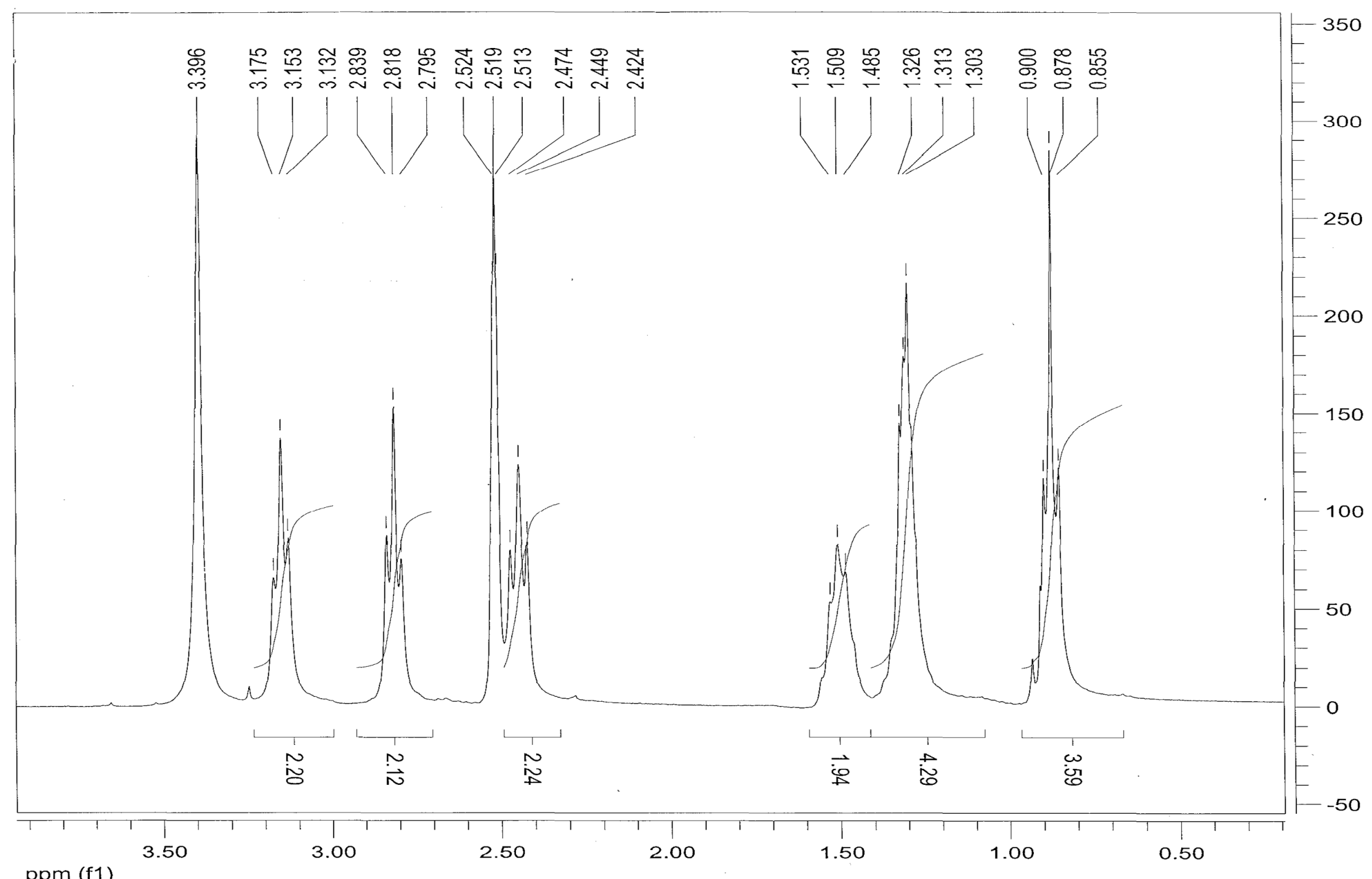


Figure S11. ${ }^{1} \mathrm{H}$ NMR spectrum of $23 \mathrm{a}$ in $\mathrm{d}_{6}$-DMSO at $30^{\circ} \mathrm{C}$.

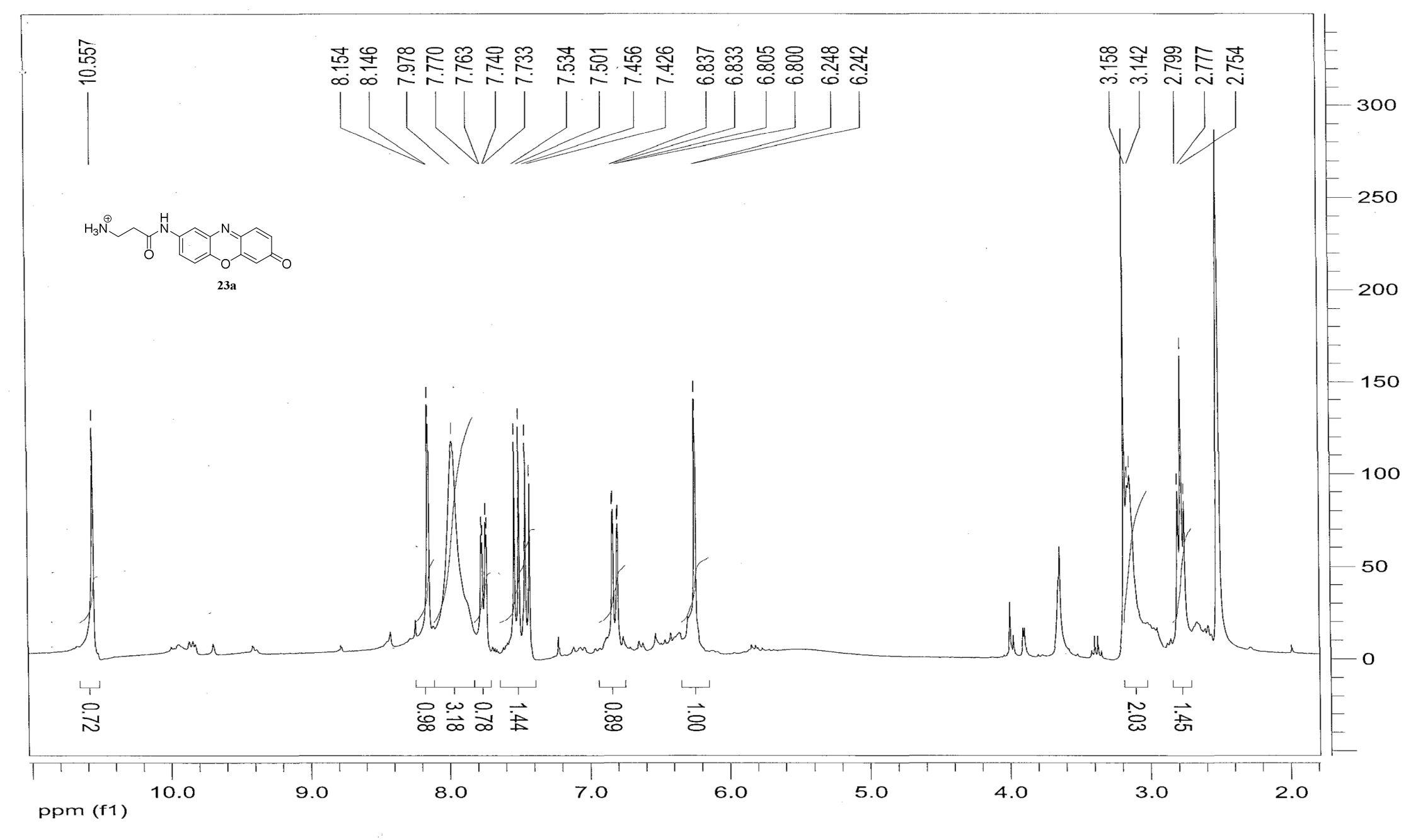




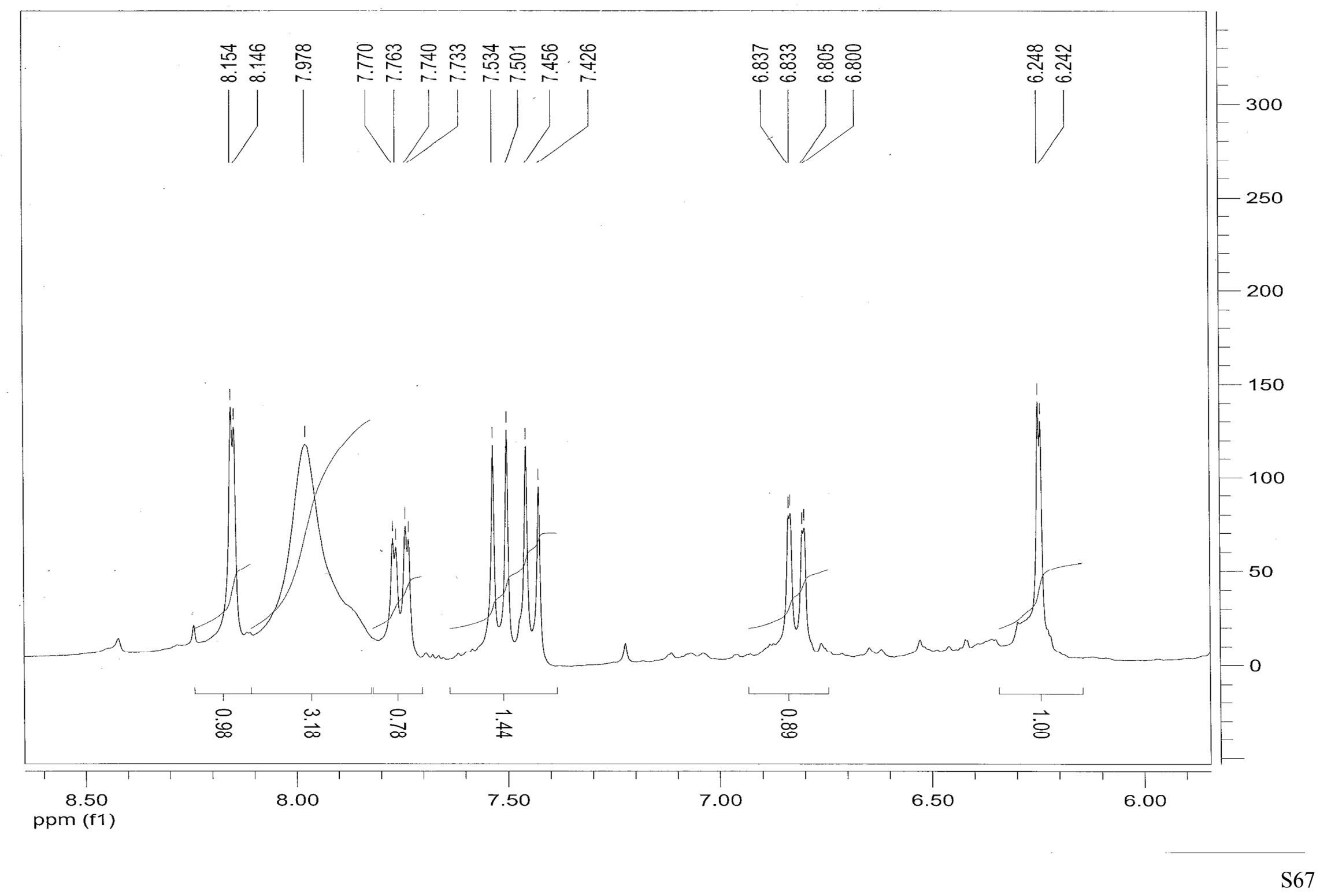




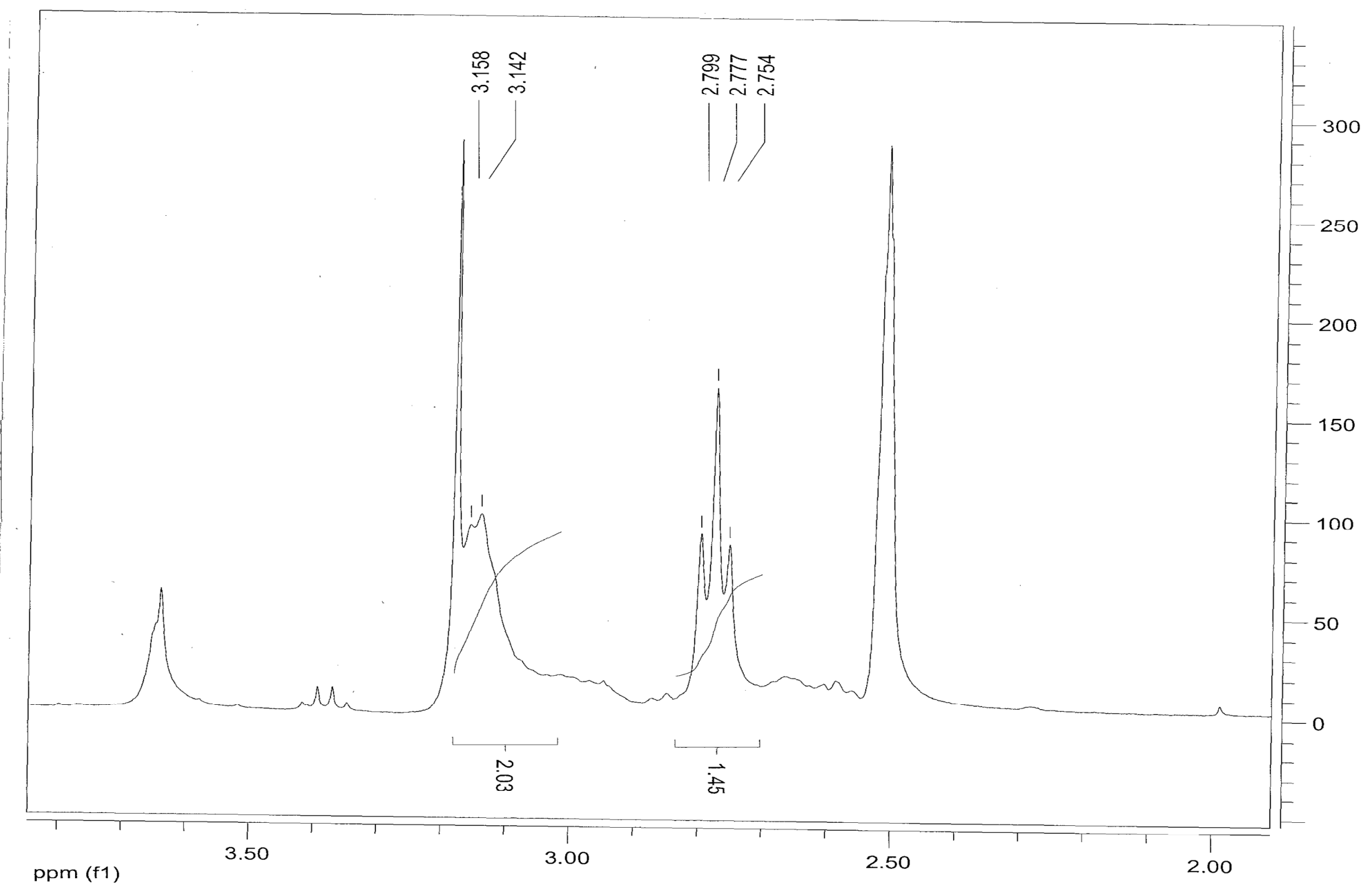


Figure S12. ${ }^{1} \mathrm{H}$ NMR spectrum of $\mathbf{2 3 b}$ in $\mathrm{d}_{6}$-DMSO at $30^{\circ} \mathrm{C}$.

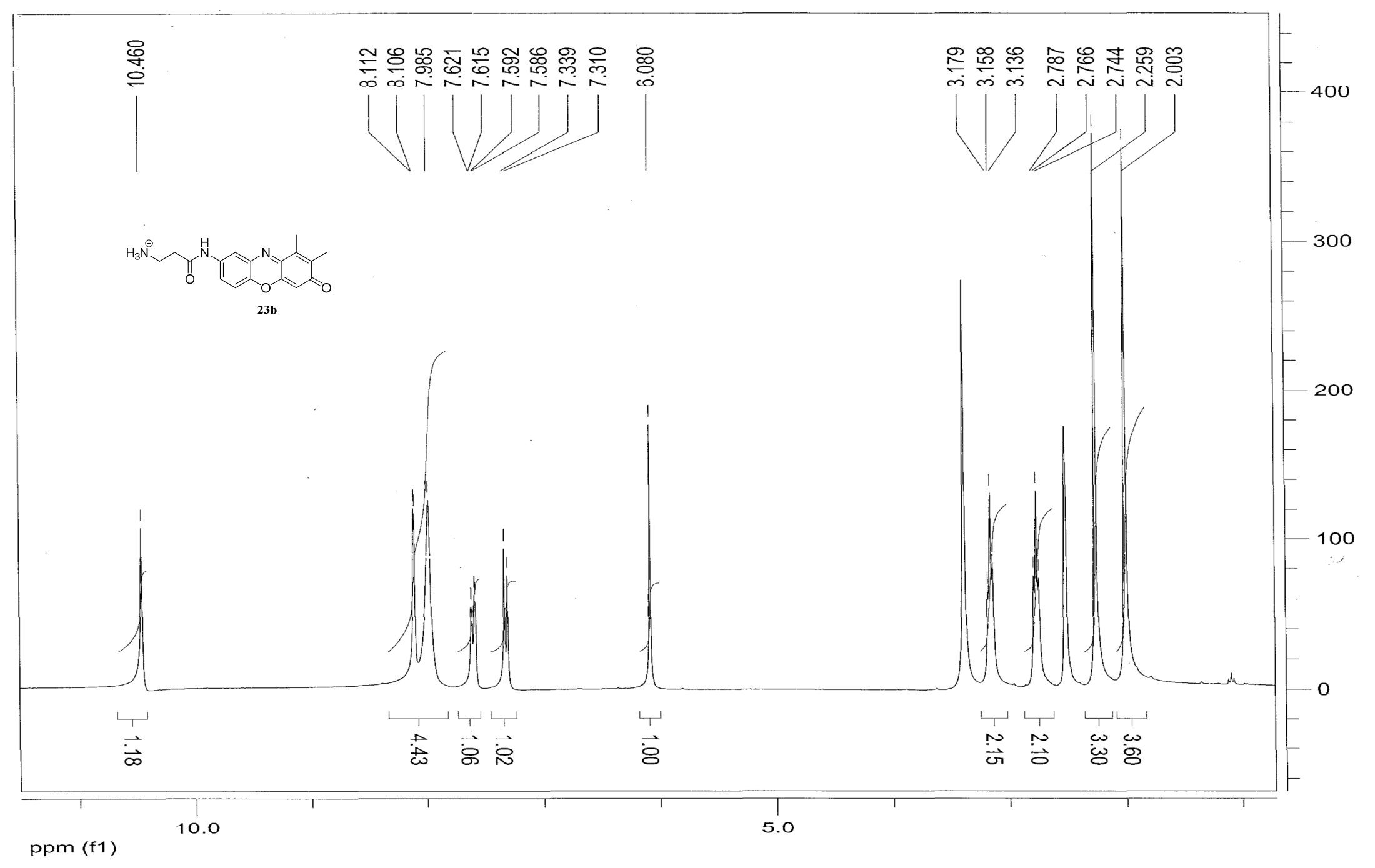




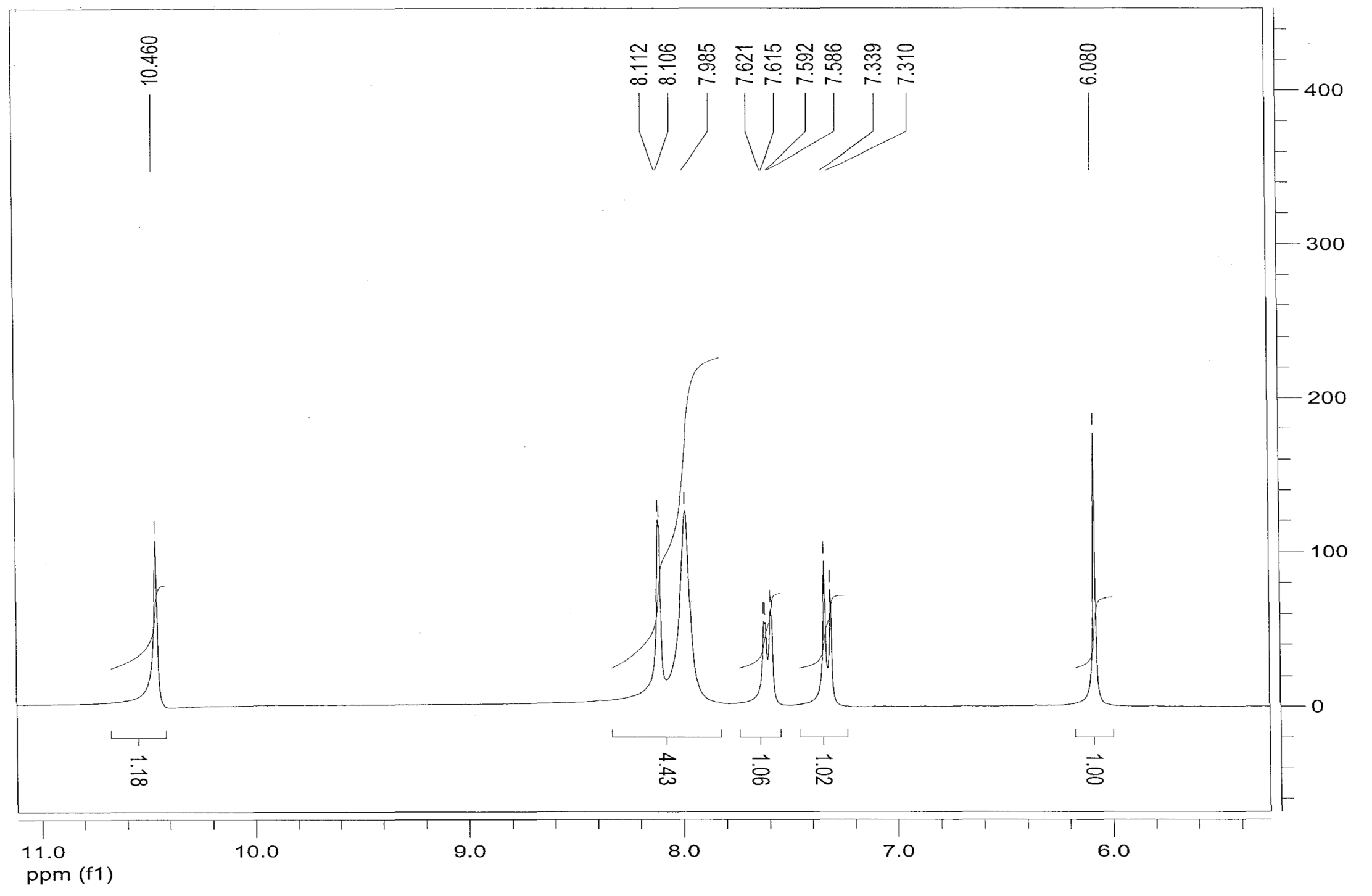




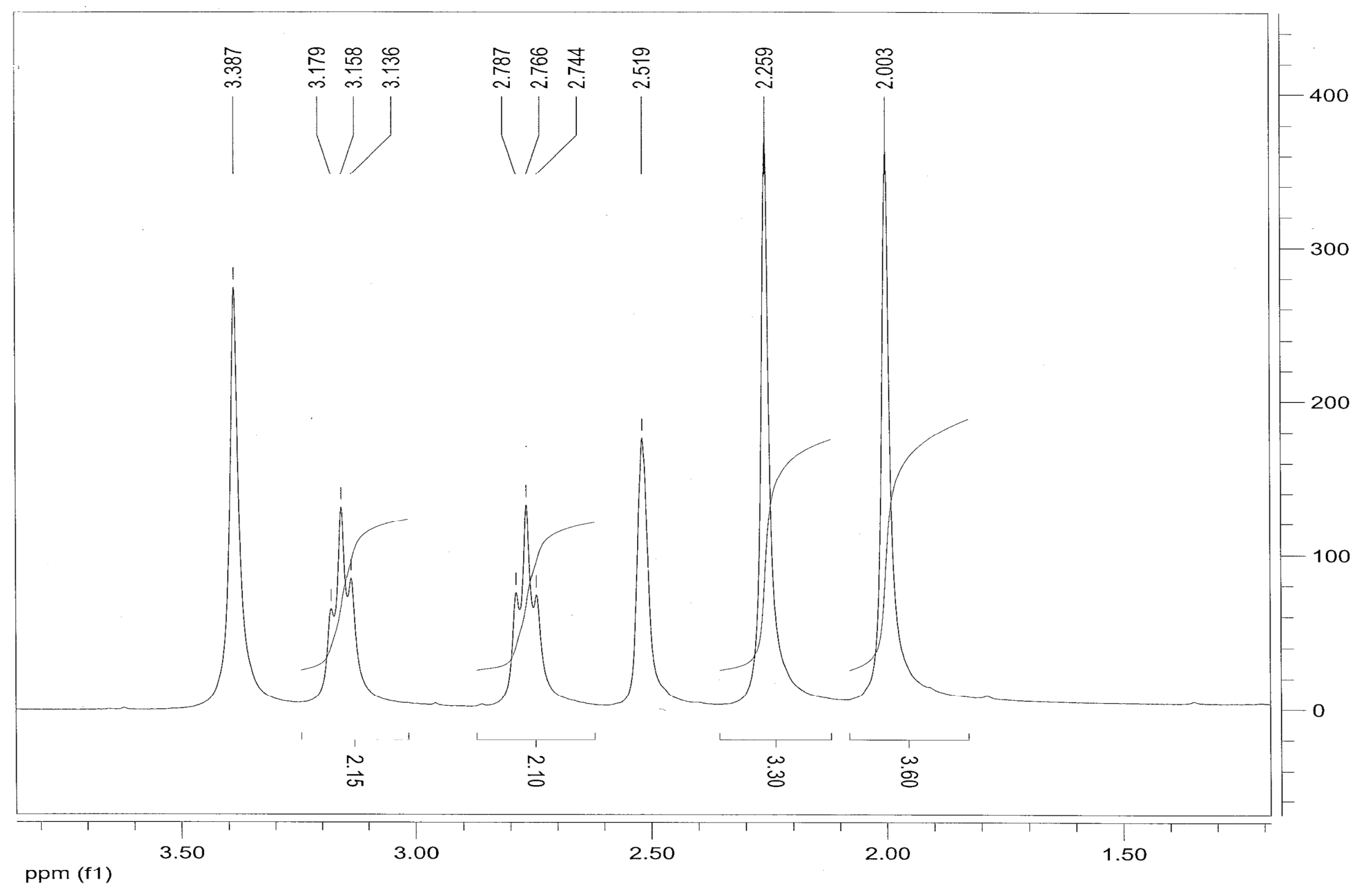


Figure S13. ${ }^{1} \mathrm{H}$ NMR spectrum of $\mathbf{2 3 c}$ in $\mathrm{d}_{6}-\mathrm{DMSO}$ at $30^{\circ} \mathrm{C}$.

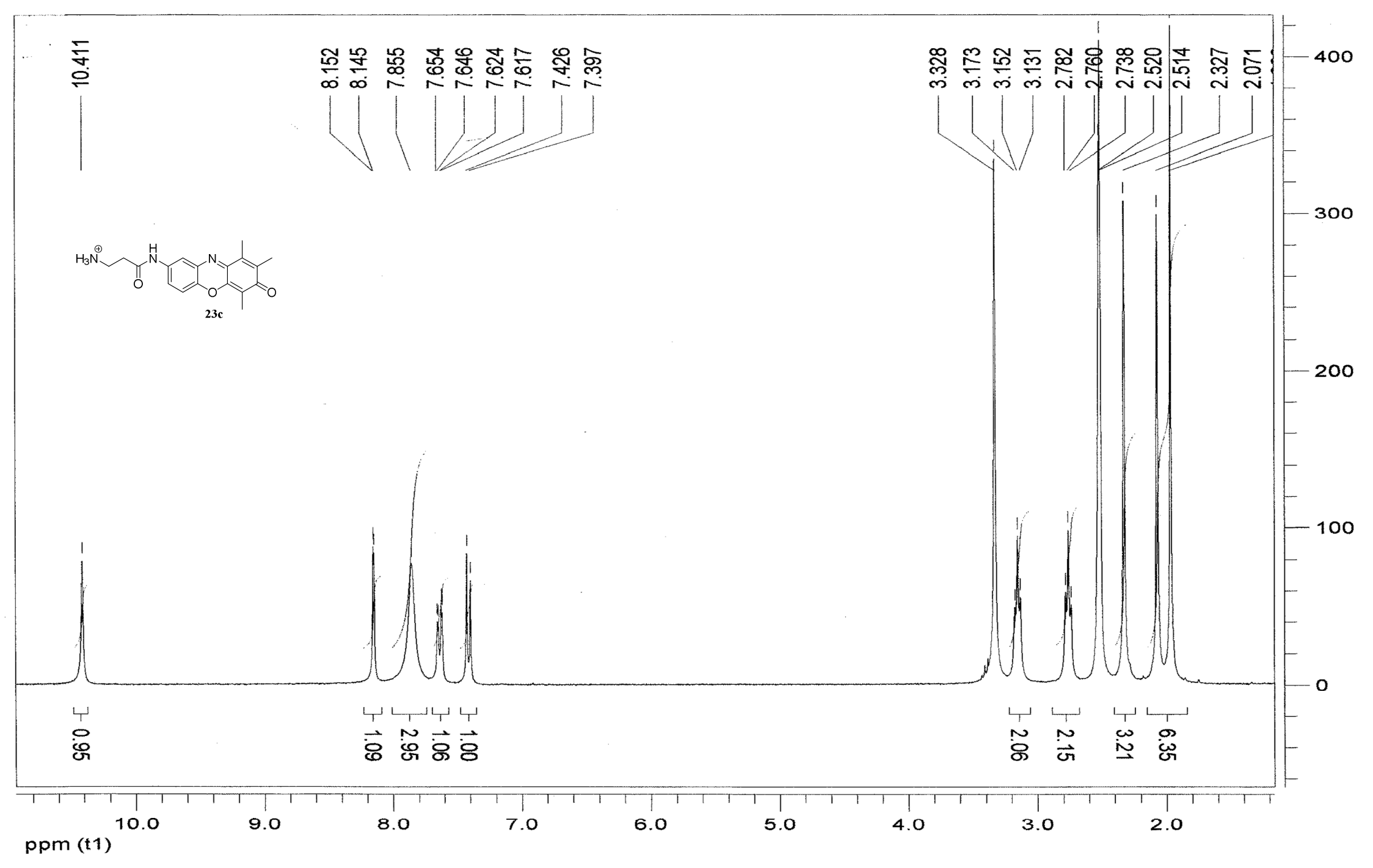




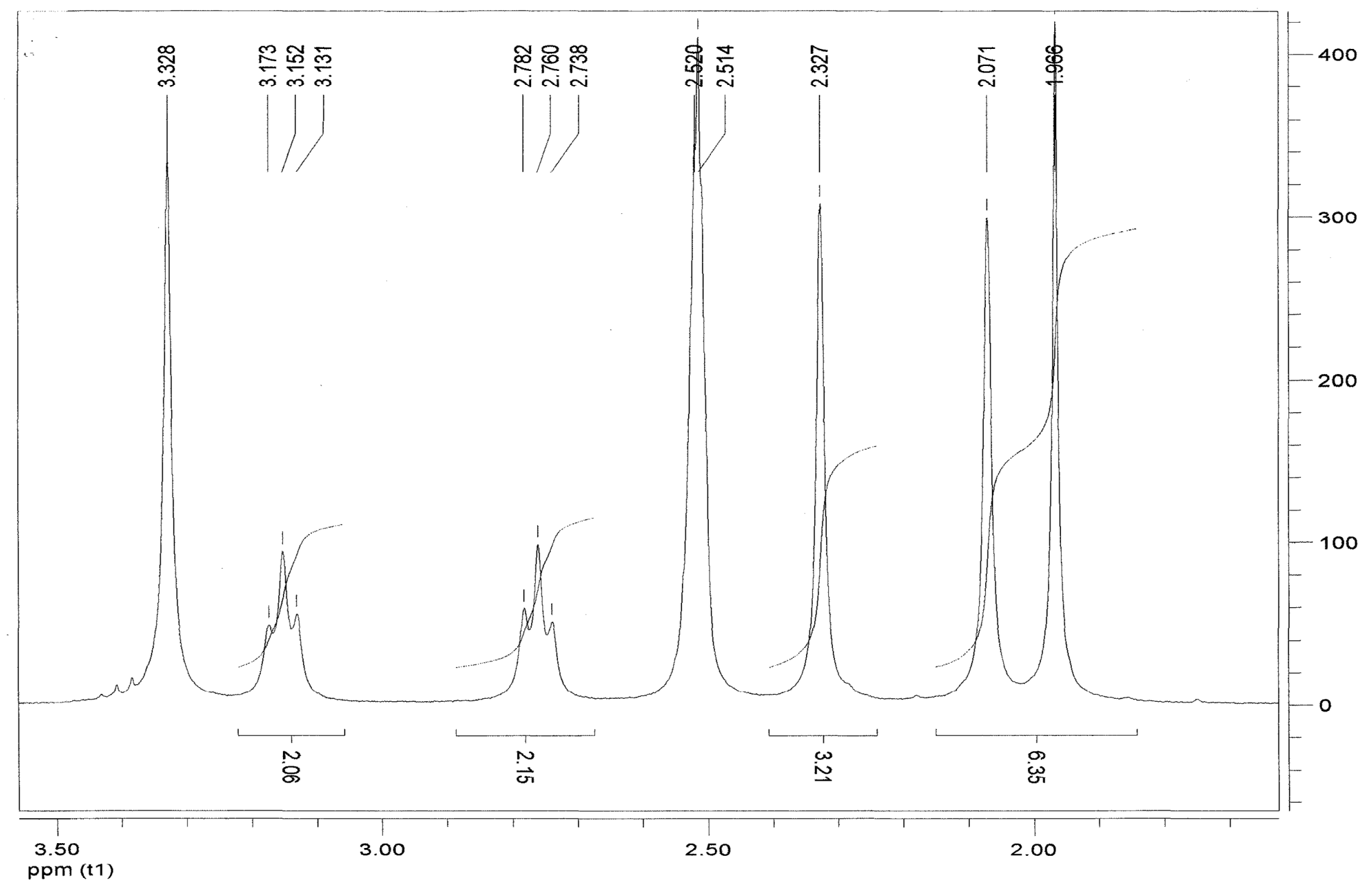


Figure S14. ${ }^{1} \mathrm{H}$ NMR spectrum of $23 \mathrm{~d}$ in $\mathrm{d}_{6}$-DMSO at $30^{\circ} \mathrm{C}$.

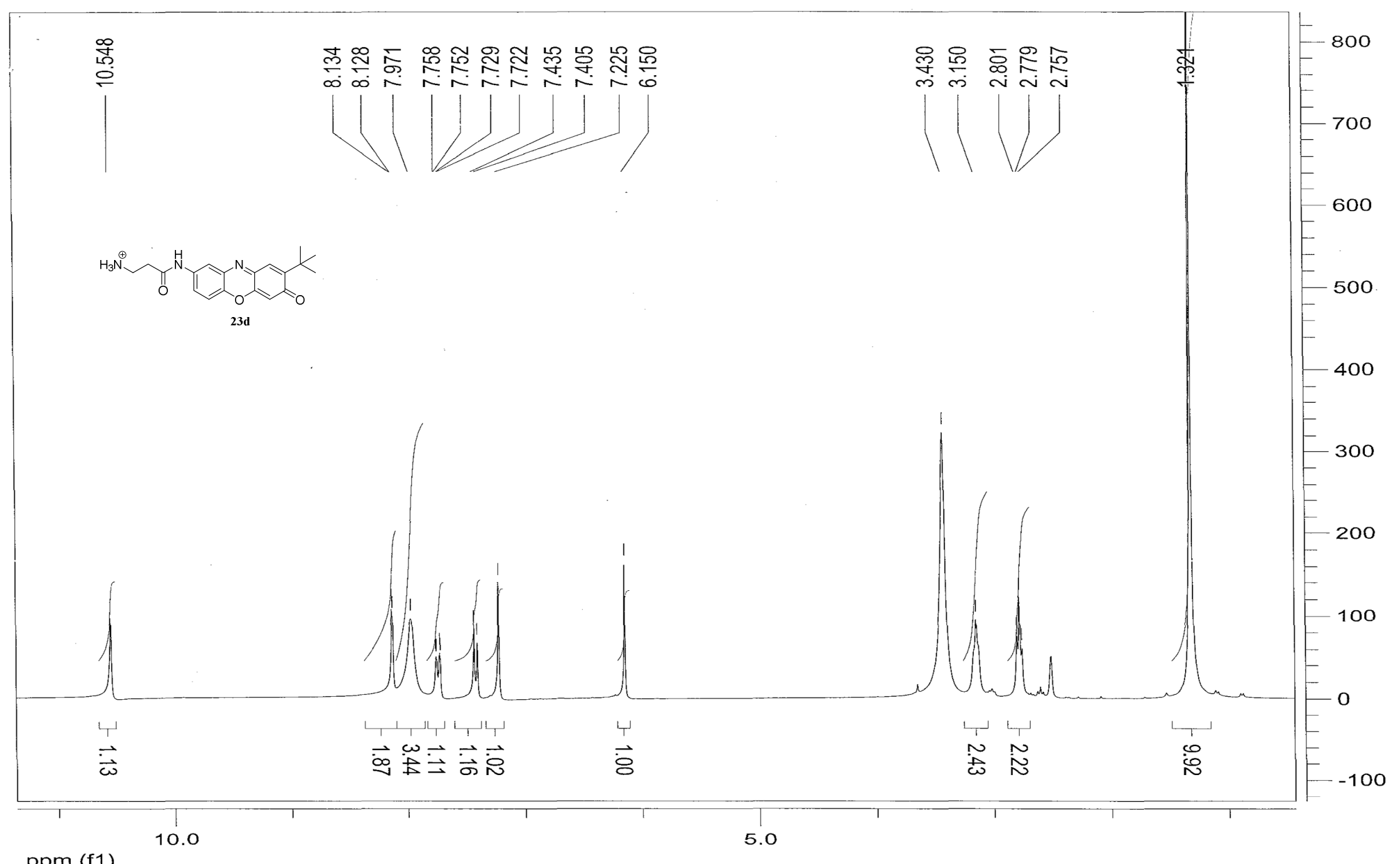

ppm (f1) 


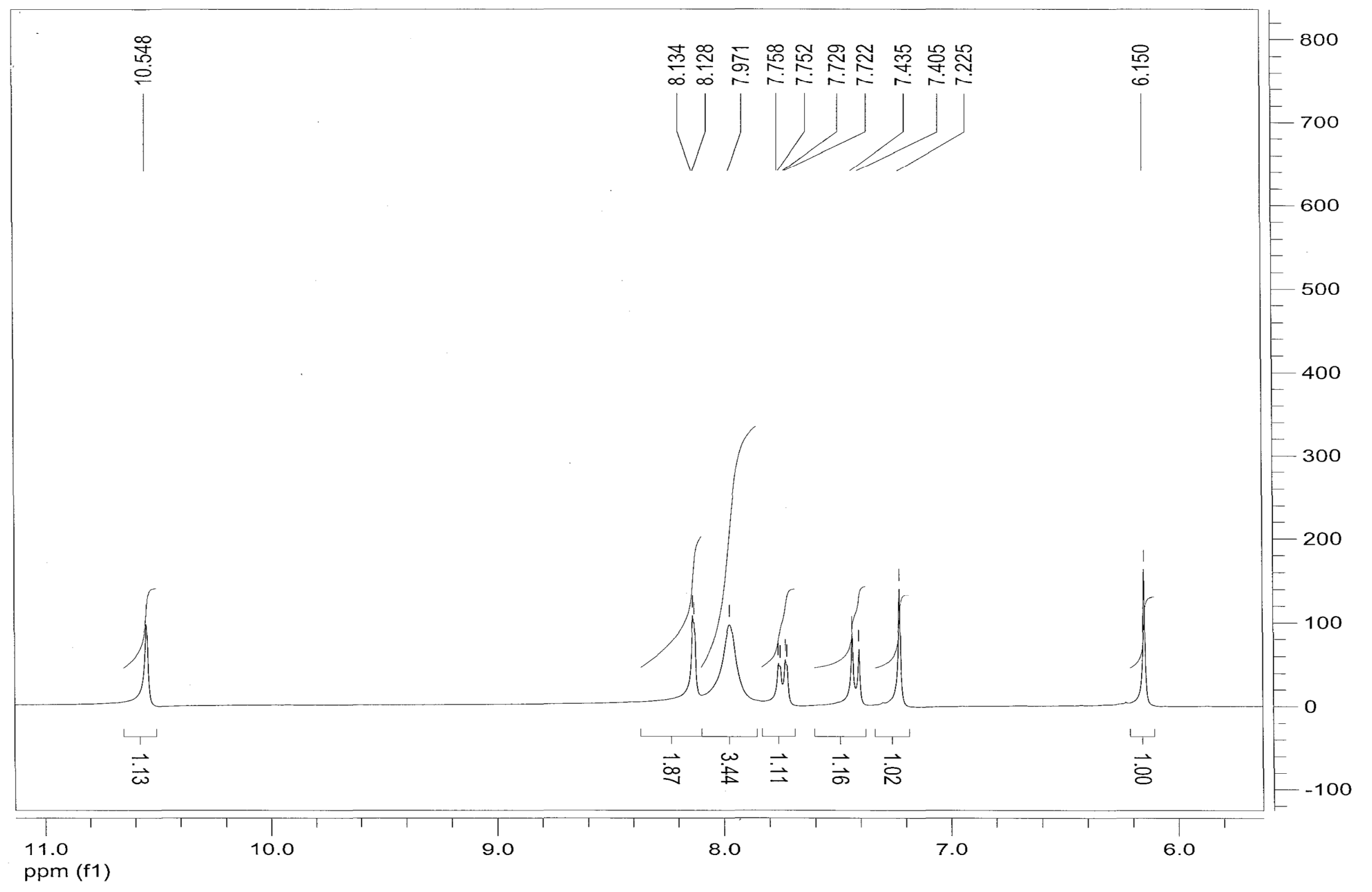




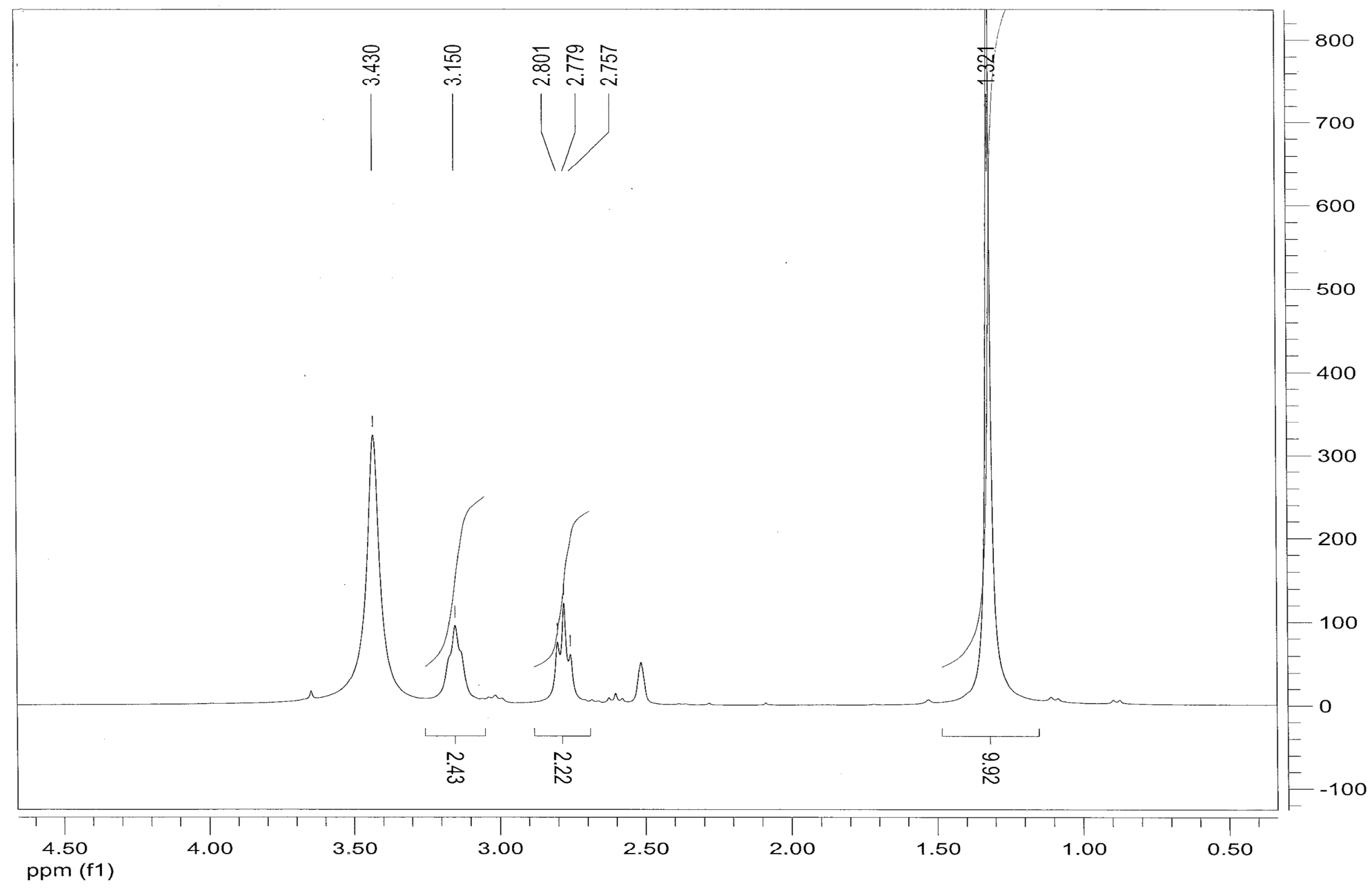


Figure S15. ${ }^{1} \mathrm{H}$ NMR spectrum of $23 \mathrm{e}$ in $\mathrm{d}_{6}$-DMSO at $50^{\circ} \mathrm{C}$.

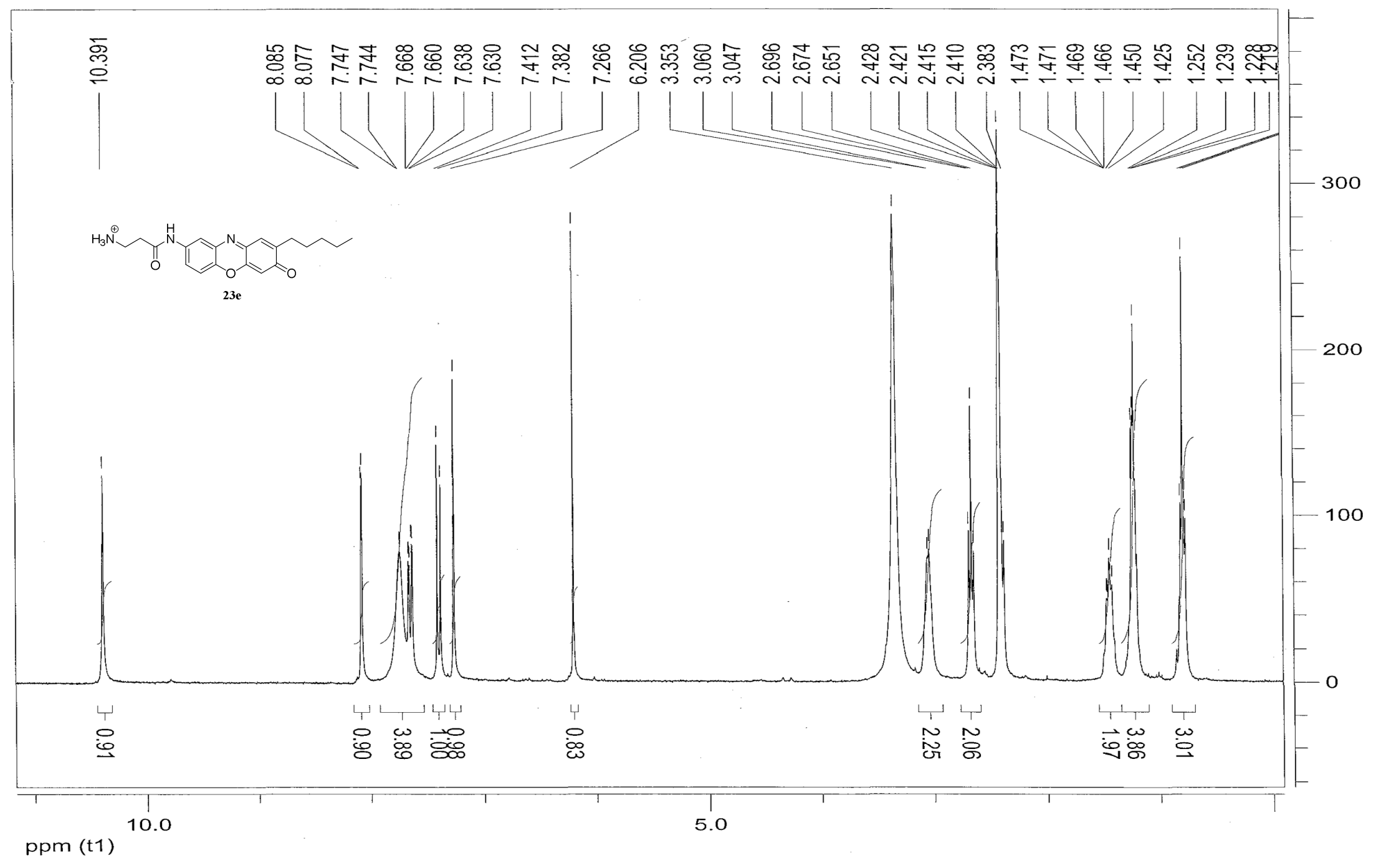




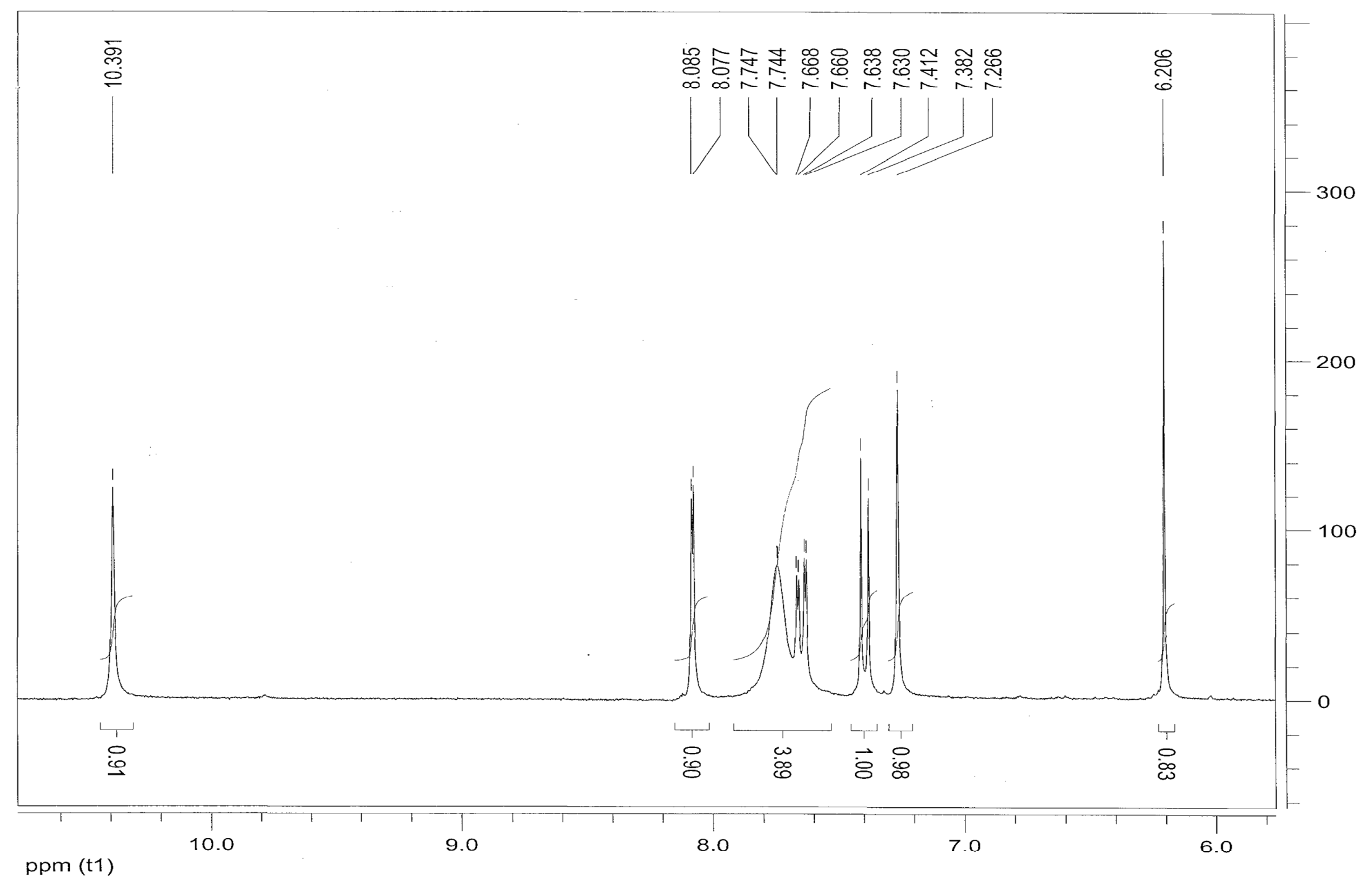




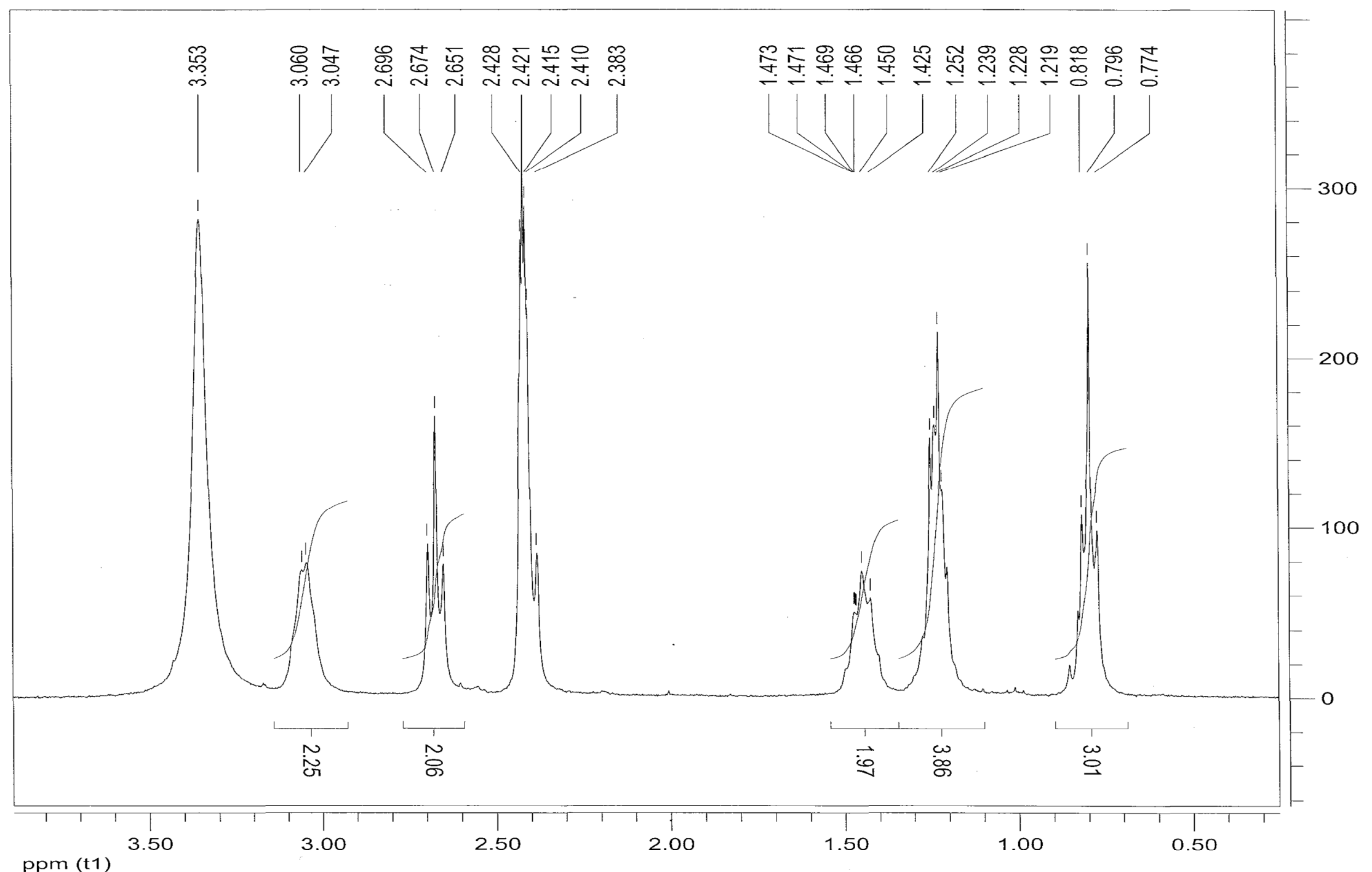


Figure S16. ${ }^{1} \mathrm{H}$ NMR spectrum of $\mathbf{2 3 f}$ in $\mathrm{d}_{6}-\mathrm{DMSO}$ at $50^{\circ} \mathrm{C}$.

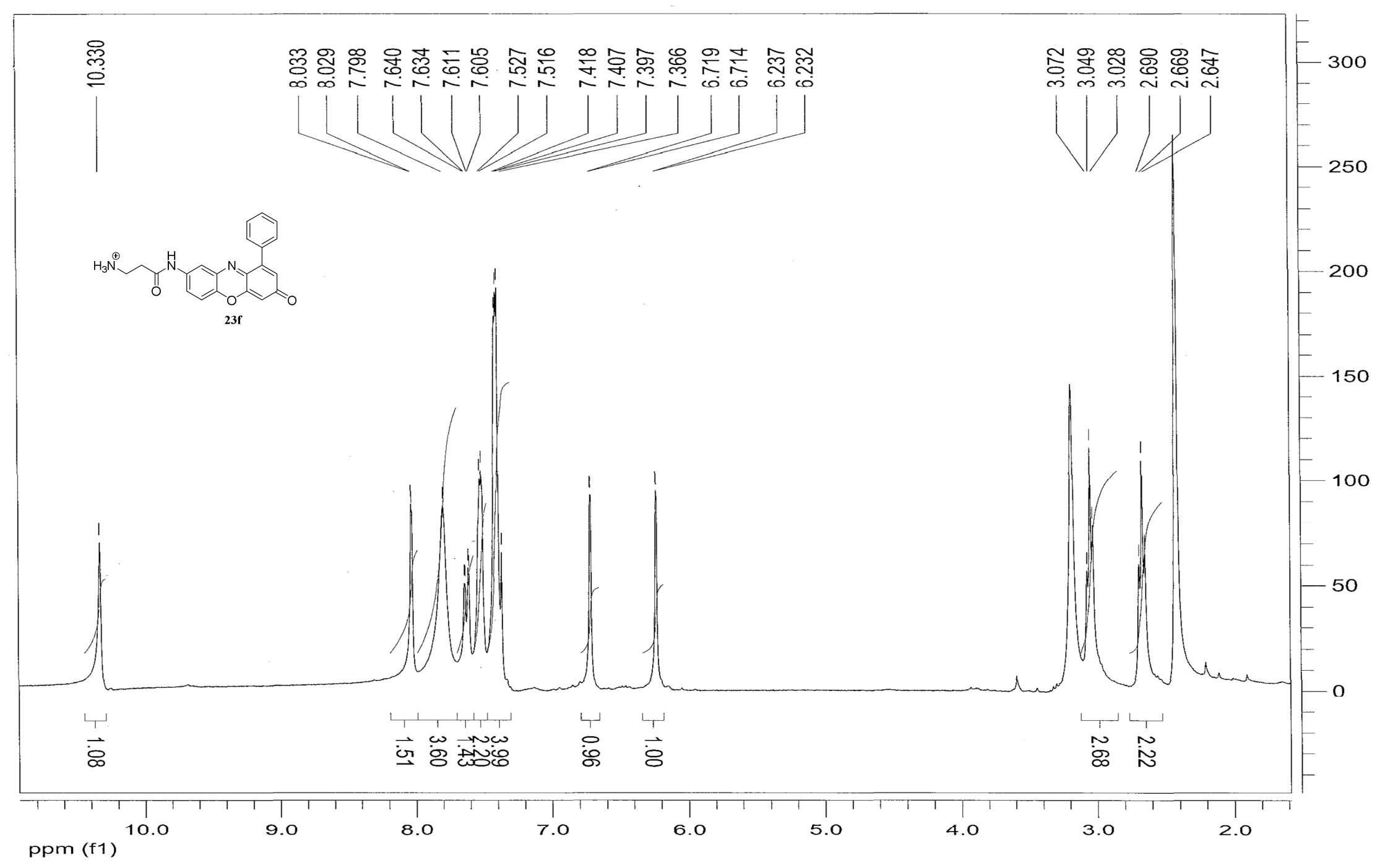




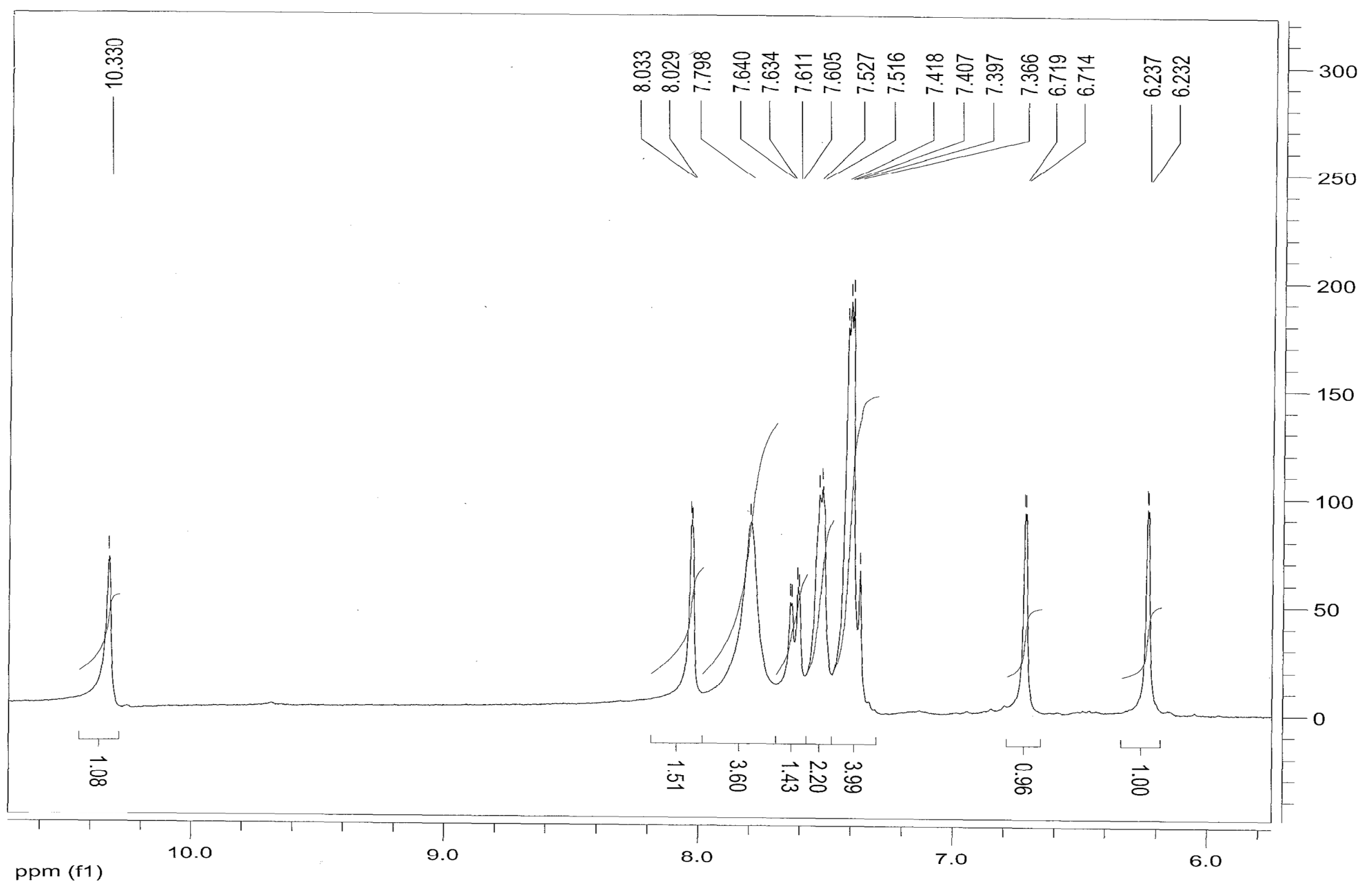




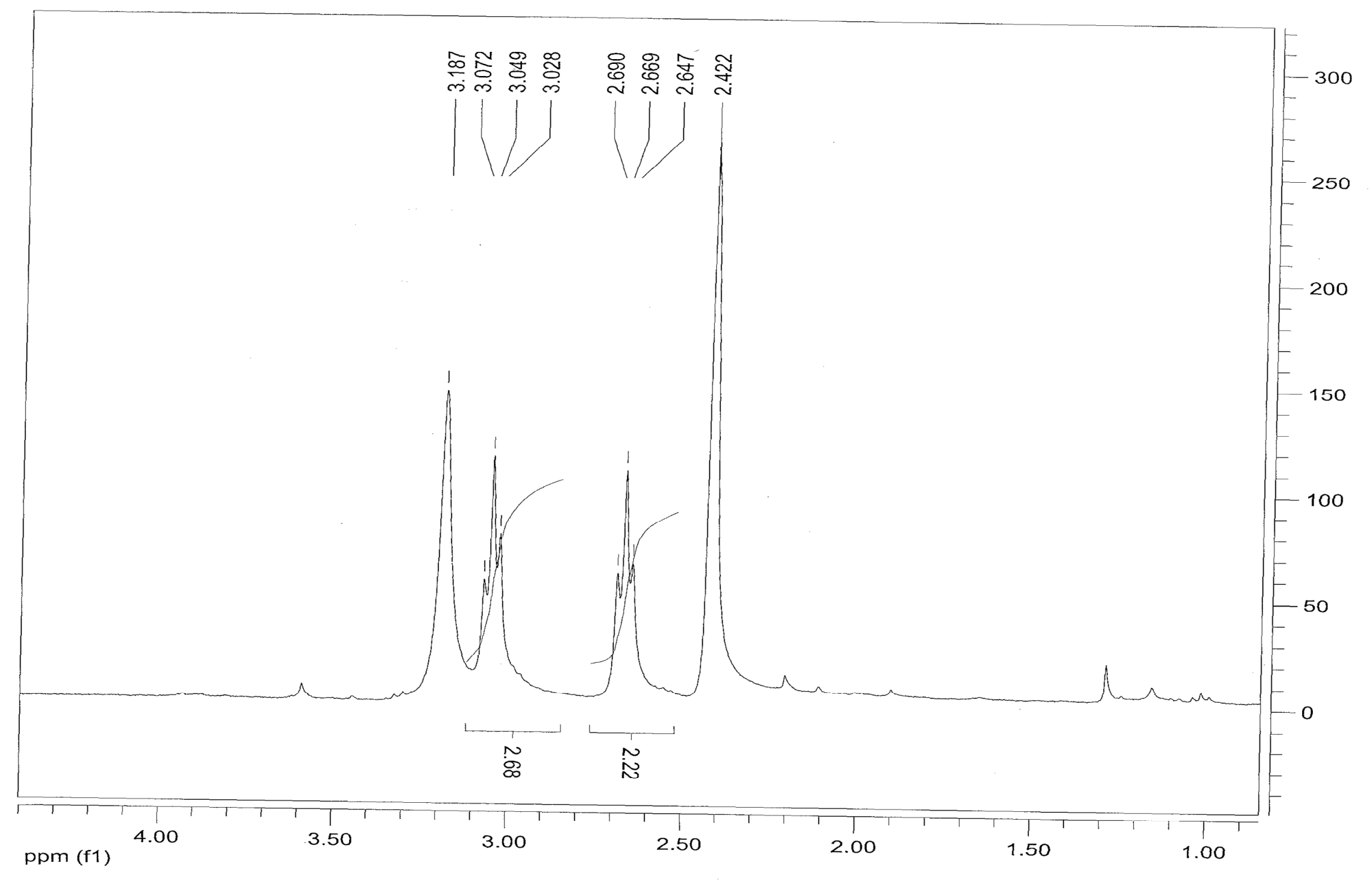

